NBER WORKING PAPER SERIES

\title{
EXPANDING THE MEASUREMENT OF CULTURE WITH A SAMPLE OF TWO BILLION HUMANS
}

\author{
Nick Obradovich \\ Ömer Özak \\ Ignacio Martín \\ Ignacio Ortuño-Ortín \\ Edmond Awad \\ Manuel Cebrián \\ Rubén Cuevas \\ Klaus Desmet \\ Iyad Rahwan \\ Ángel Cuevas \\ Working Paper 27827 \\ http://www.nber.org/papers/w27827
NATIONAL BUREAU OF ECONOMIC RESEARCH
1050 Massachusetts Avenue
Cambridge, MA 02138
September 2020

\begin{abstract}
A.C. acknowledges funding from the European Union's Horizon 2020 innovation action program under grant agreement No 786741 (SMOOTH project); and the Ministerio de Economía, Industria y Competitividad, Spain, and the European Social Fund (EU), under the Ramón y Cajal program (Grant RyC-2015-17732). R.C. acknowledges funding from H2020 EU Project PIMCITY (Grant 871370) and the Taptap Digital-UC3M Chair in Advanced AI and Data Science applied to Advertising and Marketing. I.O. acknowledges funding from ECO2013-42710-P, MDM 2014-0431 and Fundación BBVA. I.M. acknowledges funding from Spanish Ministry of Education under the FPU program (FPU15/03518). The authors thank Niccolo Pescetelli and Alex Rutherford for their helpful comments. The views expressed herein are those of the authors and do not necessarily reflect the views of the National Bureau of Economic Research.
\end{abstract}

NBER working papers are circulated for discussion and comment purposes. They have not been peer-reviewed or been subject to the review by the NBER Board of Directors that accompanies official NBER publications.

(C) 2020 by Nick Obradovich, Ömer Özak, Ignacio Martín, Ignacio Ortuño-Ortín, Edmond Awad, Manuel Cebrián, Rubén Cuevas, Klaus Desmet, Iyad Rahwan, and Ángel Cuevas. All rights reserved. Short sections of text, not to exceed two paragraphs, may be quoted without explicit permission provided that full credit, including $\odot$ notice, is given to the source. 
Expanding the Measurement of Culture with a Sample of Two Billion Humans

Nick Obradovich, Ömer Özak, Ignacio Martín, Ignacio Ortuño-Ortín, Edmond Awad, Manuel

Cebrián, Rubén Cuevas, Klaus Desmet, Iyad Rahwan, and Ángel Cuevas

NBER Working Paper No. 27827

September 2020

JEL No. C80,J10,J16,O10,R10,Z10

\section{$\underline{\text { ABSTRACT }}$}

Culture has played a pivotal role in human evolution. Yet, the ability of social scientists to study culture is limited by the currently available measurement instruments. Scholars of culture must regularly choose between scalable but sparse survey-based methods or restricted but rich ethnographic methods. Here, we demonstrate that massive online social networks can advance the study of human culture by providing quantitative, scalable, and high-resolution measurement of behaviorally revealed cultural values and preferences. We employ publicly available data across nearly 60,000 topic dimensions drawn from two billion Facebook users across 225 countries and territories. We first validate that cultural distances calculated from this measurement instrument correspond to traditional survey-based and objective measures of cross-national cultural differences. We then demonstrate that this expanded measure enables rich insight into the cultural landscape globally at previously impossible resolution. We analyze the importance of national borders in shaping culture, explore unique cultural markers that identify subnational population groups, and compare subnational divisiveness to gender divisiveness across countries. The global collection of massive data on human behavior provides a high-dimensional complement to traditional cultural metrics. Further, the granularity of the measure presents enormous promise to advance scholars' understanding of additional fundamental questions in the social sciences. The measure enables detailed investigation into the geopolitical stability of countries, social cleavages within both small and large-scale human groups, the integration of migrant populations, and the disaffection of certain population groups from the political process, among myriad other potential future applications.

Nick Obradovich

Center for Humans and Machines

Max Planck Institute for Human Development

Lentzeallee 94

14195 Berlin

Germany

obradovich@mpib-berlin.mpg.de

Ömer Özak

Department of Economics

Southern Methodist University

3300 Dyer Street

Suite 301, Umphrey Lee Center

Box 0496

Dallas, TX 75275-0496

omer@omerozak.com
Ignacio Martín

Nommon Solutions and Technologies

Calle Claudio Coello 124

Madrid 28006

Spain

ignacio.martin@nommon.es

Ignacio Ortuño-Ortín

Department of Economics

Universidad Carlos III

28903 Getafe

Madrid

Spain

iortuno@eco.uc3m.es 
Edmond Awad

Department of Economics

University of Exeter

Business School Rennes Drive

Exeter EX4 4PU

United Kingdom

e.awad@exeter.ac.uk

Manuel Cebrián

Center for Humans and Machines

Max Planck Institute for Human Development

Lentzeallee 94

Berlin 14195

Germany

cebrian@mpib-berlin.mpg.de

Rubén Cuevas

Department of Telematic Engineering

and UC3M-Santander Big Data Institute

Avenida de la Universidad 30

Leganés (Madrid) 28911

Madrid

rcuevas@it.uc3m.es

Klaus Desmet

Department of Economics

and Cox School of Business

Southern Methodist University

3300 Dyer, Suite 301

Dallas, TX 75205

and CEPR

and also NBER

kdesmet@smu.edu

Iyad Rahwan

Center for Humans and Machines

Max Planck Institute for Human Development

Lentzeallee 94

14195 Berlin

Germany

rahwan@mpib-berlin.mpg.de

Ángel Cuevas

Department of Telematics Engineering

and UC3M-Santander Big Data Institute

Universidad Carlos III de Madrid

Avenida de la Universidad 30

Leganés (Madrid) 28911

Spain

acrumin@it.uc3m.es 
Culture has played a pivotal role in human evolution ${ }^{1-4}$, setting our species apart from the rest of the animal world. As a result, the study of human culture is one of the core endeavors of the social sciences. Tens of thousands of scientists around the world study culture ${ }^{5}$, with disciplines ranging from anthropology ${ }^{6,7}$ to sociology ${ }^{8}$, from political science ${ }^{9-11}$ to economics, ${ }^{12-14}$ and from psychology ${ }^{15-19}$ to philosophy ${ }^{20}$. Their work has enabled the understanding of many human social, economic, and political phenomena ${ }^{12,21-28}$, and serves as a bedrock of knowledge in the social sciences.

The traditional quantitative approach to the study of culture has been shaped by the historical availability of data ${ }^{29}$. For most of the history of the study of culture, collecting empirical data on humans has been costly, time consuming, and in many instances impossible ${ }^{30}$. As a result, scholars tend inductively to distill their broad theoretical definitions of culture from the top down ${ }^{29}$, producing a set of cultural constructs measurable via surveys in data constrained environments. Cultural dimensions that result from this process tend to be a select few salient and measurable features of human groups such as artistic and culinary practices ${ }^{31}$, language ${ }^{32,33}$ and literature ${ }^{34}$, political ideologies ${ }^{35}$ and institutions ${ }^{23}$, and religions and religious practices ${ }^{36}$.

Whereas a scholar of culture historically would often need to directly observe individuals in the field to produce a rich description of their cultural attributes ${ }^{37}$, the information age has enabled measuring certain aspects of human life at high resolution globally and unobtrusively ${ }^{30,38}$. Humans today spend an ever increasing amount of time on devices that continuously track and record users' interests, beliefs, preferences, behaviors, locations and interactions. The data produced from this process are massive in size, extensive in coverage, and high in resolution.

This changing information environment enables supplementing and expanding the scientific approach to the measurement of culture. Here we propose new methods for the bottom-up measurement of culture globally. We first explore the strengths and weaknesses of traditional quantitative approaches to the measurement of culture and show their relation to our method. We then measure culture from the bottom up and examine our measure's performance against traditional quantitative measures. Finally, we investigate cultural questions previously impossible to examine without the combined resolution and scope our method enables. Ultimately, high-resolution granular data are essential for our understanding of many cultural phenomena, ranging from wars and the formation of identity to the integration of immigrants and the fragmentation of societies.

Traditional quantitative approaches to the study of culture benefit from numerous strengths. For example, these studies of culture are often relatively low in dimension and therefore readily measurable via quantitative surveys. This parsimony has enabled excellent studies of certain cultural features in highly data constrained settings $s^{27,39,40}$. Traditional approaches also provide substantial face validity: they focus on many of the concepts typically associated with culture. These traditional approaches, as a result, likely encapsulate many important constituent cultural constructs. A final benefit of the parsimony of traditional approaches is that scholars from a wide variety of fields can measure and study different aspects of culture.

Yet while the traditional approach to culture has a number of benefits, it also has various weaknesses. First, a critical question when attempting to construct a broad measure of human culture from the top down is: where do we draw the line in terms of what to measure? Surely religion should be considered part of culture. But is group-level support for a football team part of culture? What about preferences for video games ${ }^{41}$ or television shows? What about group-level appreciation of cat videos or the color of socks that we choose to wear? A scholar may determine religion - but not preference for a television show - to be the more theoretically 
important constituent of culture to measure based on the argument that television preferences are not central to the human experience. However, another scholar with a differing opinion could argue that, given the amount of time humans in the developed world spend watching television ${ }^{42}$, the choice of particular show reflects an important implicit value of the humans that watch it. If a line about what to measure must be drawn from the top down, scholars will very likely reasonably disagree on where, precisely, it should fall. However, the very attempt to narrow cultural constructs from the top down highlights the second weakness of this measurement strategy. The justification for excluding any particular construct is necessarily endogenous to the particular culture (and cultural bias) of the scientist(s) doing the theorizing. Crucially, where to draw this line is a direct function of the cultural preferences of each scholar's particular human group. This endogeneity problem arises for every supervised attempt to include or exclude a concept from the measurement of culture.

Third, the parsimonious nature of top-down approaches presents its own limitations. Implicitly, traditional quantitative approaches to the study of culture tend to focus on features that provide insight into differences among human groups ${ }^{43-49}$. Yet, human groups may be similar in many more dimensions than they are dissimilar. Top-down definitions tend to occlude these dimensions of similarity with an implicit focus on those features - the arts, language, politics, religion, and distinct traditions - that differentiate human groups.

Finally, traditional quantitative measures of culture commonly rely on self-reported answers to survey questions $^{50}$ or subjective evaluations of the particular scientists conducting the study ${ }^{51}$. These pose measurement challenges. Questions such as: "Is religion important to you?" or "Do you think adultery is immoral?" pose substantial risks of social desirability bias ${ }^{52}$ and direct observation of subjects induces risk of Hawthorne and experimenter demand effects ${ }^{53}$, among other related methodological concerns.

Here we expand the measurement of culture from the bottom up, enabling a rich, unobtrusive, quantitative description of global cultural factors. Inspired by ethnographic methods ${ }^{54}$, we take culture as the set of information and attributes that characterizes a human group at a point in time. We can conceive of the culture of a human group at a point in time as a complex, high-dimensional hypersurface (the black surface in Figure 1A). This surface is not narrowed from the top down. Conceptually, it includes art and Angry Birds appreciation, ethics and email enjoyment, formalities and football fans, language and loungewear likes, religion and running routines, politics and potluck preferences, and social structures and sockwear, among every other feature of human life.

Our approach enables measuring culture from the bottom up (the blue surface in Figure 1A), providing a complement to traditional top-down approaches (the red surface in Figure 1A). Importantly, it prioritizes no features over others and enables the data to reveal important dimensions among observed human groups. This allows our method to be general, flexible, and unsupervised. And it does not selectively exclude constructs, reducing the biases in the measurement of culture encountered by top-down methods.

But how does one go about measuring the high-dimensional hypersurface of culture? Private firms have - perhaps unintentionally - led the way. Effectively and precisely targeting products and services to individuals requires gathering massive amounts of information about those individuals' beliefs, behaviors, and preferences $^{55-57}$. For online companies like Google and Facebook, the gathering, storing, and selling of this information has itself become a multi-billion dollar enterprise. As a result, the platforms have become adroit at measuring myriad features of human lives via activities that occur during the large amount of time modern humans spend both online and offline in proximity to connected devices ${ }^{58}$. 

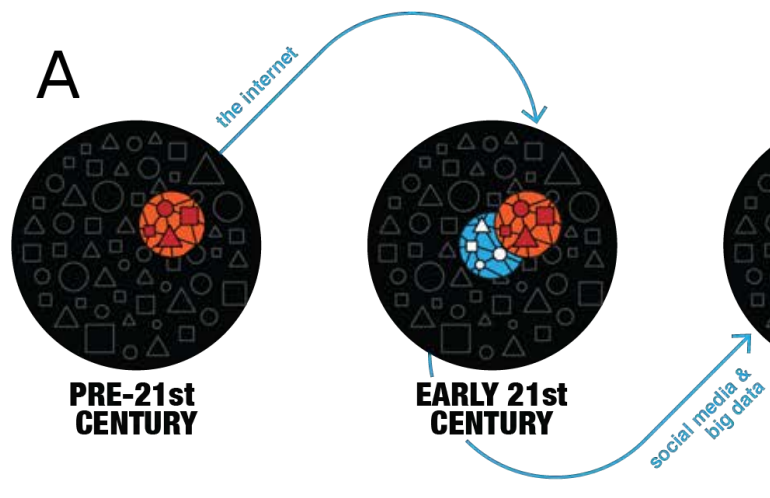

\section{B ACTIVITY ON}
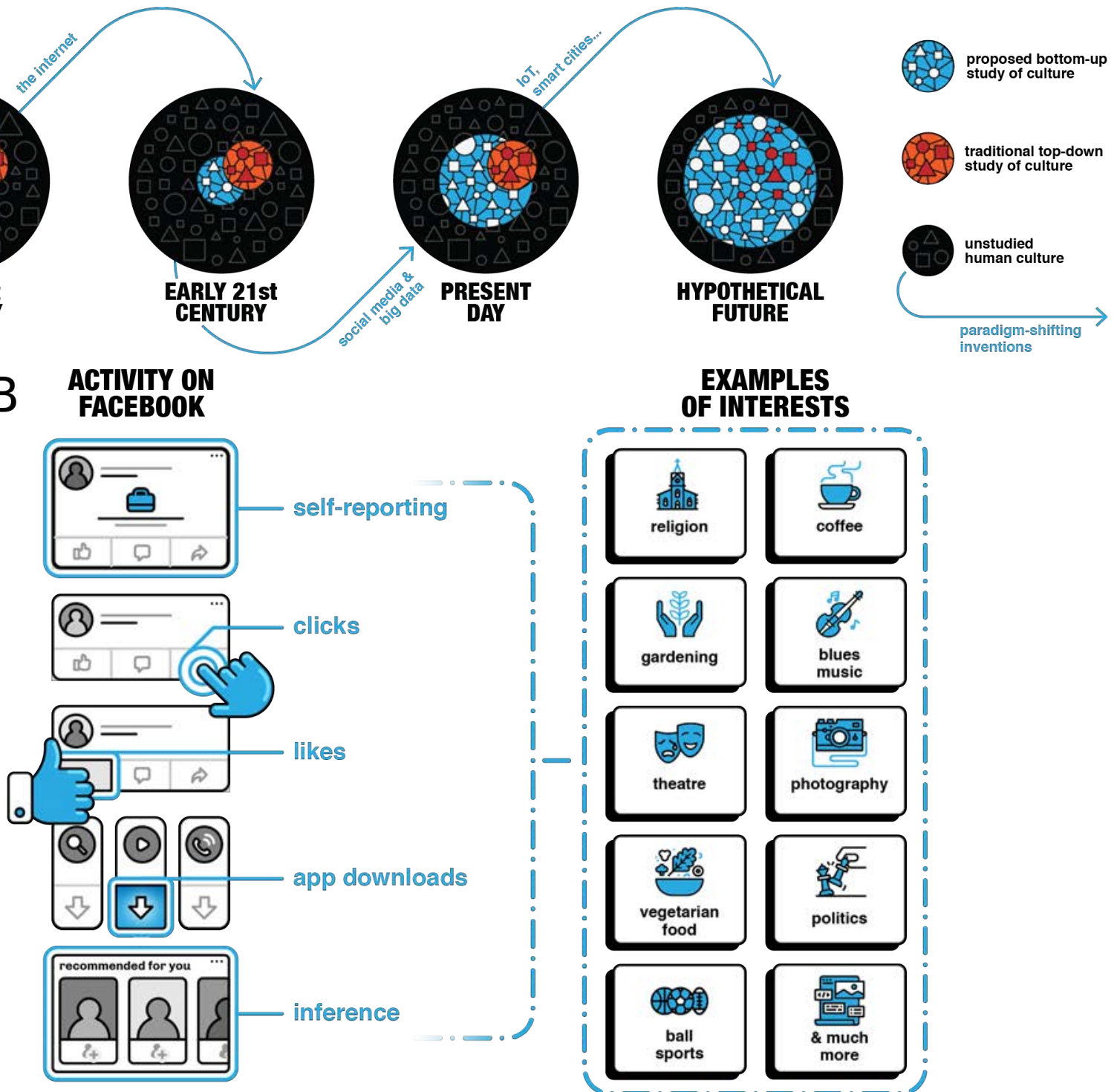

\section{EXAMPLES OF INTERESTS}

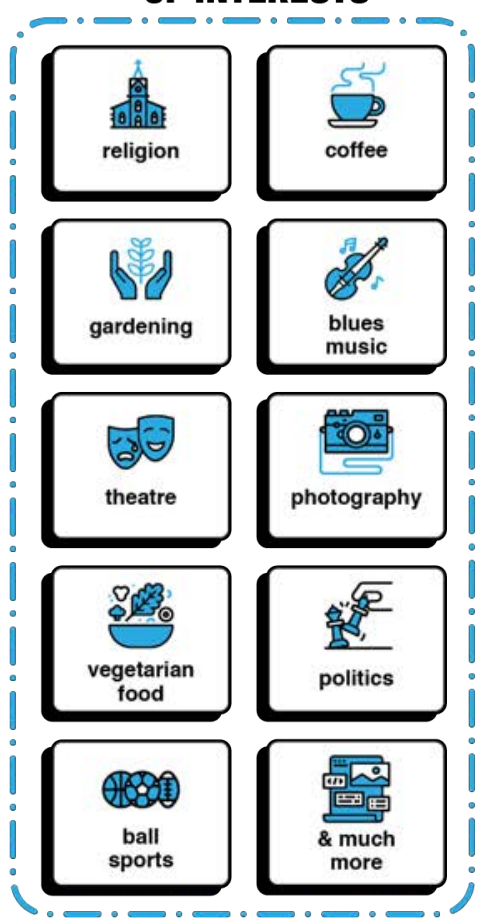

Figure 1: Measurement of the cultural landscape and methods of classification of values, behaviors, preferences, and interests on Facebook. Panel A illustrates how the bottom-up quantitative study of culture is enabled by information technology advances and the broad measurement of humans across the globe. Paradigm shifting technologies such as the Internet, the advent of social media and big data, the introduction of the Internet of Things (IoT) and smart cities all shape the availability of information with which to measure previously unstudied dimensions of culture. We anticipate a hypothetical future in which traditional top-down concepts of culture are encompassed by and integrated into a bottom-up approach to the measurement of culture. Panel B illustrates how Facebook classifies users' interests via users' self reporting, via users' observed behavior which includes the totality of users' clicks on the platform and on ads served elsewhere by the platform, via users' 'likes' and software downloads, and via broad inference based on users' overall behavior on and off the platform. The interests Facebook infers span hundreds of thousands of dimensions and include topics that both fall within more traditional measures of culture - such as religion, politics, and the arts - as well as those that tend to fall outside of traditional measures - such as interests in video games, physical activity preferences, and recreational drug interests. 
Facebook places particular importance in classifying the interests of its users ${ }^{59}$. As a result, the company has inadvertently built the largest platform for the measurement of culture in existence (see Figure 1B). Fortunately for scholars, Facebook makes this information accessible to prospective marketers via a marketing Application Programming Interface (API). Using information drawn from users' self-reported interests, clicking behaviors on Facebook, likes on Facebook, software downloads, GPS location, behavior on other sites that employ Facebook ads (Figure 1B), this API provides the ability to create and analyze social groups of interest along hundreds of thousands of interest dimensions and down to very fine spatial and temporal resolution (the zip code-by-day level in the US). Table S4 illustrates examples of cultural categories along with corresponding Facebook interests both for traditional and non-traditional cultural elements. By making its platform open to those interested in marketing to its users, Facebook has enabled scholars to interrogate its measures of global human interests and construct freely available measures of culture.

We use data gleaned from scraping the Facebook Marketing API to construct a high-dimensional measure of culture. We gathered nearly 60,000 diverse interests by sequentially interrogating Facebook's platform and then constructed - for each administrative unit in our analysis - a vector of the share of individuals in that unit that held each interest (see Methods for added detail). Importantly, each interest on the platform is indexed by a unique identifier, allowing for consistency across languages globally. We use these data to investigate culture at the country, subnational, and local levels.

Employing data on these interest shares drawn from over two billion individual users around the world, we first validate our measure of culture derived from Facebook interests employing country differences from traditional means of measuring cultural distance ${ }^{60}$. If our Facebook measure captures important components of traditional top-down measures, we should expect to observe a positive correspondence between our bottom-up measure and traditional top-down measures (see Methods for a description of distance and correlation measures).

Figure 2A presents the results of these comparisons. Our bottom-up measure of inter-country cultural distance corresponds positively and significantly to a wide variety of typical measures of cultural distance between countries. We observe small positive correlations between our measure and measures of linguistic, geographic, religious, and genetic distance between country populations ${ }^{61-70}$ (see Methods). However, between more direct measures of traditional notions of culture - provided via the World Values Survey (WVS) ${ }^{51,71}$ - we observe a more marked positive correspondence with a correlation coefficient of approximately 0.5 (coefficient: 0.54 , p-value: 0.0001 ). Thus our bottom-up measure of cultural distance corresponds positively but imperfectly to traditional measures.

Does this imperfect correspondence result from the measurement of additional components of cultural distance between countries? At face value, Figure 2B shows that the number of Facebook interests are several orders of magnitude larger than the number of questions in the WVS. To further investigate whether this also translates into capturing more dimensions of culture, we perform principal component analysis on Facebook interests and WVS questions, using the common sample of 69 countries covered by both data sources (see Methods). Our goal is to reduce the dimensionality of interests and questions and to assess how many unique principal components are able to explain a large share (80\%) of the variance in our Facebook measure of culture and in the WVS questions across countries.

Figure $2 \mathrm{C}$ plots the share of the overall variance in questions and interests that is explained by principal 


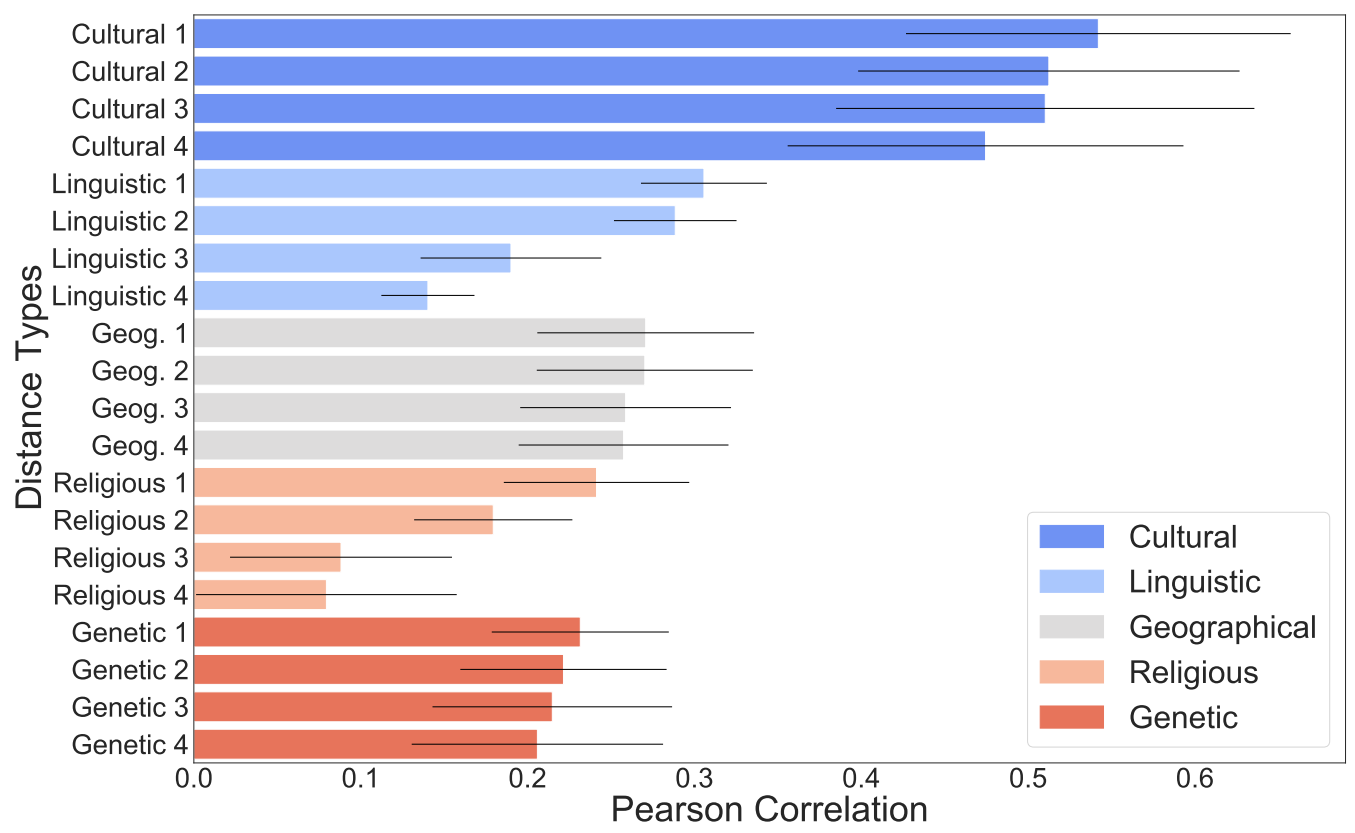

(a) Correlation between Facebook and Traditional Measures

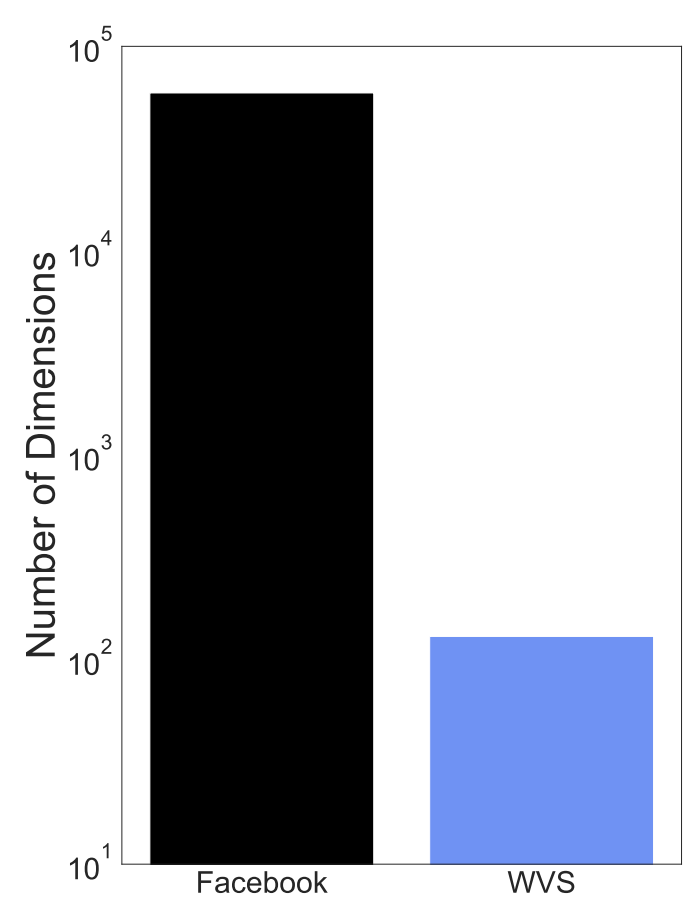

(b) Number of Dimensions

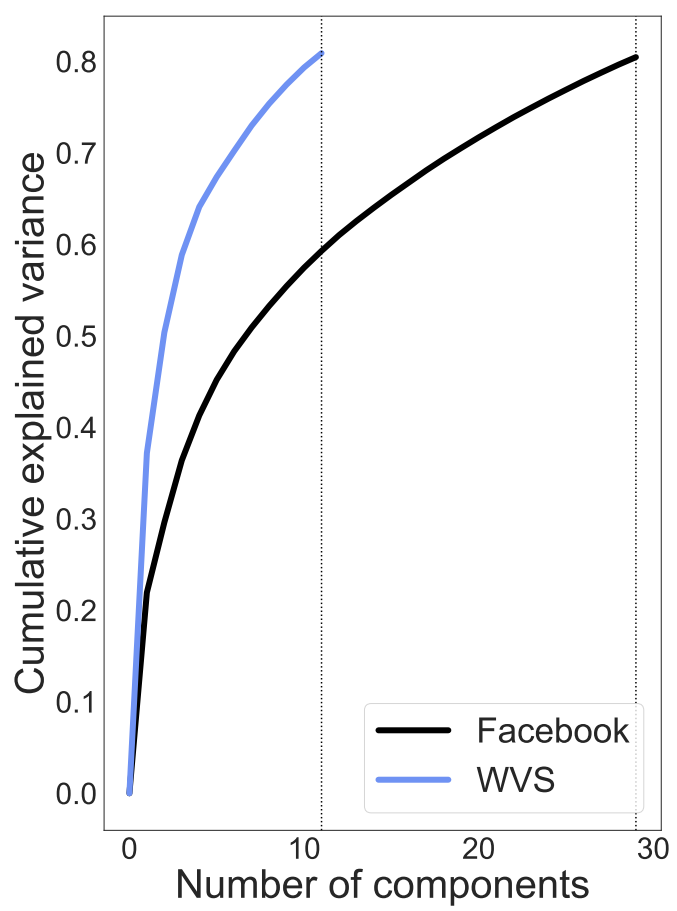

(c) Principal Component Analysis

Figure 2: Bottom-up measurement of culture correlates with traditional top-down measures, enables the use of orders of magnitude more features, and explains additional variance. (A) Correlation between our bottom-up Facebook inter-country cultural distance measure and inter-country cultural distances based on traditional proxies (see Methods for full list). Bars show the Pearson correlation coefficient between our measure and the four most highly correlated measures within each type of conventional proxy (genetic, geographical, linguistic, religious, values). Black lines depict the $95 \%$ confidence interval for the correlation coefficient based on Mantel tests. Results are based on the common sample of 69 countries for which all measures are available. (B) Bars show the number of Facebook interests and the number of common questions included in all waves of the World Values Survey. (C) Lines depict the number of principal components and their cumulative explained variance for each measure. To explain $80 \%$ of the variation underlying the Facebook interests data across countries 29 principal components are required, as opposed to the 11 principal components necessary to explain the same level of variation in the WVS. 


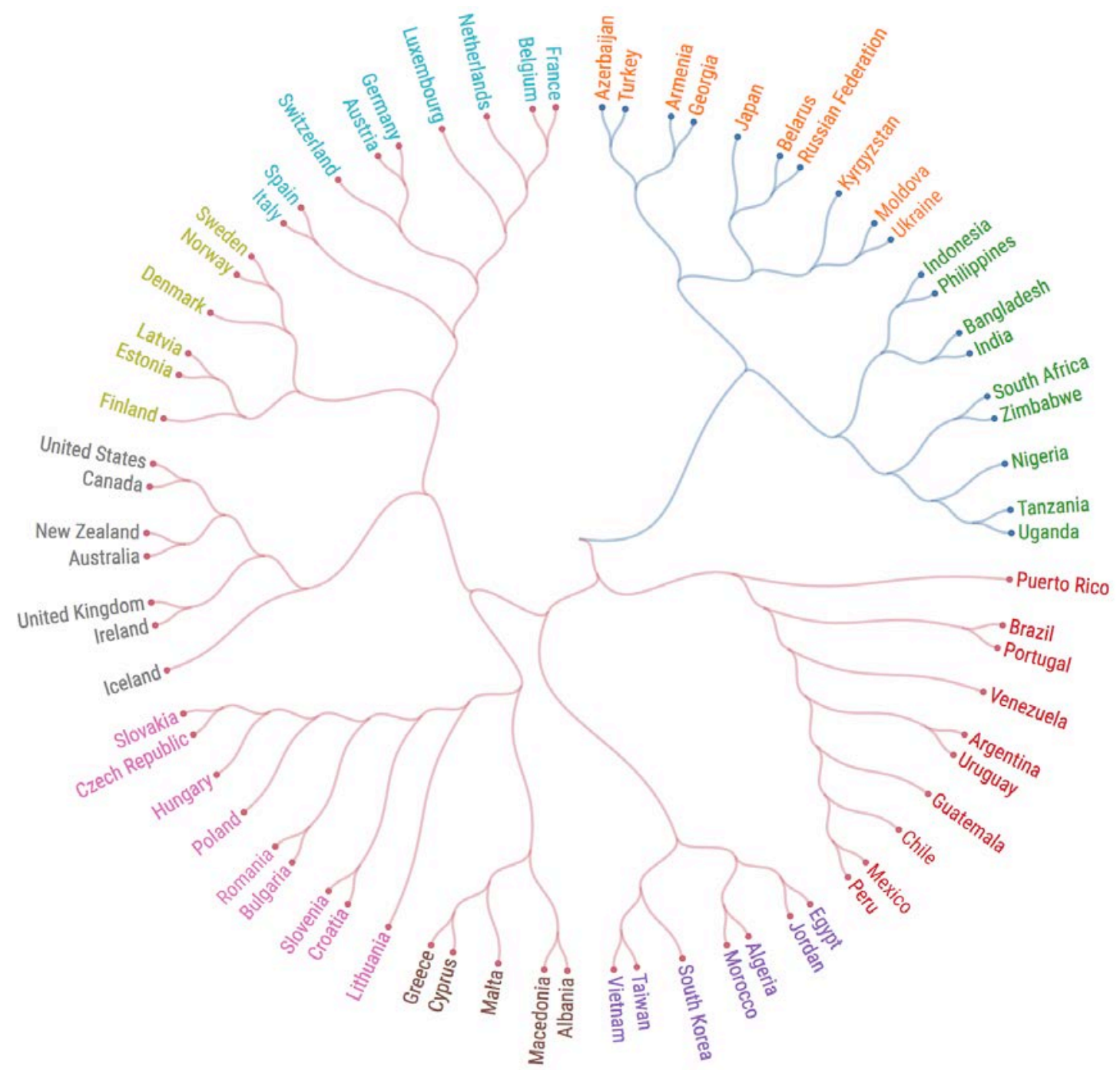

Figure 3: Bottom-up measurement of culture returns sensible clusters of countries. Dendrogram is generated using cosine distance and the Ward linkage method (see Methods). Countries and territories with at least 300,000 persons, a Facebook penetration rate of more than 5\%, and representation in both the Facebook and WVS data were selected. The color of a country's link represents its membership to a main cluster, while the color of its name represents its membership to a sub-cluster. Two countries of the same color are closer to each other than to a country of a different color.

components as a function of their number. Our measure of culture derived from Facebook interests explains $80 \%$ of the variance between countries using three times the number of principal components as required to explain $80 \%$ of the variance using the WVS. This provides suggestive evidence that the Facebook measure covers a more diverse array of explanatory dimensions of culture as compared to the WVS.

While our Facebook data span a broad variety of interests, do they also capture a broader set of specific cultural traits than those measured by the WVS? To explore this question, we employ a supervised machine learning algorithm that uses all our Facebook interests to predict close to 50 specific cultural attributes, ranging from generosity to gender bias. When comparing the predicted traits to the observed traits, we find an average correlation of 0.6, indicating that the wide array of Facebook data are also able to capture specific cultural traits (see Table S3). 
Next we examine whether clusters of countries returned via our Facebook measure of culture mirror common conceptions of cultural similarity, providing a measure of face validity. Figure 3 presents a dendrogram of countries, based on the cosine distance between culture vectors constructed from our Facebook data employing the Ward linkage method. The sample of countries consists of those that overlap with the WVS, have a population of more than 300,000, and have a Facebook penetration rate of more than 5\% (see Methods). As can be seen, the unsupervised clustering of countries within our sample provides substantial validity to our measure. Countries that typically are culturally or historically associated with one another - the United States and Canada, India and Bangladesh, Germany and Austria - are placed directly next to one another in the clusters. Our approach also reveals novel features in the data that go beyond obvious geographic clustering. For example, Puerto Rico is closer to the Latin American cluster than it is to the United States, despite being a U.S. territory. Furthermore, linguistically similar but geographically disparate countries - such as the U.S. and Australia and Brazil and Portugal - cluster together. It is important to mention that clustering algorithms have some difficulty dealing with "outliers". This explains the maybe surprising location of Japan in Figure 3. However, when we extend the dendrogram to include all 225 countries, Japan is no longer special, appearing alongside China (Figure ). The Supplementary Information provides extensive robustness checks, exploring different ways of measuring distances and analyzing different samples of countries and interests.

Figures 2 and 3 provide evidence for the validity of our method. However, cultural variation is not relegated solely to nation-level groupings. Cultural differences at the subnational level are essential to understand nationbuilding efforts as well as geopolitical and secessionist threats around the globe. Unfortunately, traditional quantitative measures are highly costly to conduct and thus provide little representative insight into subnational cultures. Conducting representative surveys at high resolution globally would be cost prohibitive (in the limit, the costs would approach those of the Facebook platform itself). Might our Facebook measure be able to provide improved, scalable insight into novel subnational cultural variations?

To investigate this question, we gather vectors of Facebook interests for subnational regions in the United States and Europe and compute cosine distances between each region within the country and all the other regions within the same country (see Methods). One question is whether subnational regions are less distant to other regions within their own country than to closeby countries. Figure 4 investigates this for the regions of Spain (red), France (blue), Germany (yellow), and Italy (green). For example, panel A depicts the distribution of the cultural distances of Spanish regions to other European countries and to each other. The subnational regions within Spain are much less distant to one other than to neighboring European countries. For example, Catalonia is culturally markedly closer to any other Spanish region than to either Italy or France. This same pattern holds for regions in the other countries (Panels B-D).

Before concluding that national borders demarcate sharp cultural boundaries, we also compare cultural distances between sub-national regions in different countries. While sub-national regions are culturally closer to each other than to other countries (Paris is closer to other regions of France than to Spain), we might expect capital cities to resemble each other (Paris might be closer to Madrid than to rural regions of France). We observe the opposite. Almost all sub-regions in our data are closest to sub-regions within their own national borders. Only two sub-regions in our European data - Flanders in Belgium and Donegal County in Ireland are closer to regions in a separate country than they are to other regions in their own nation. Both exceptions can be traced back to fairly recent changes in country borders: the splitting of the province of Limburg between 


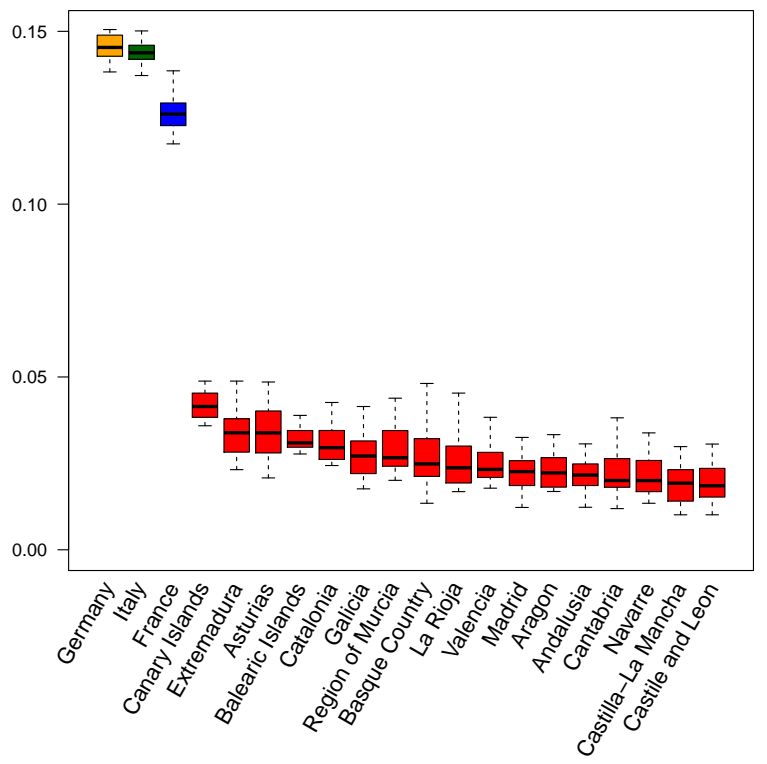

(a) Spain Regions and neighbours

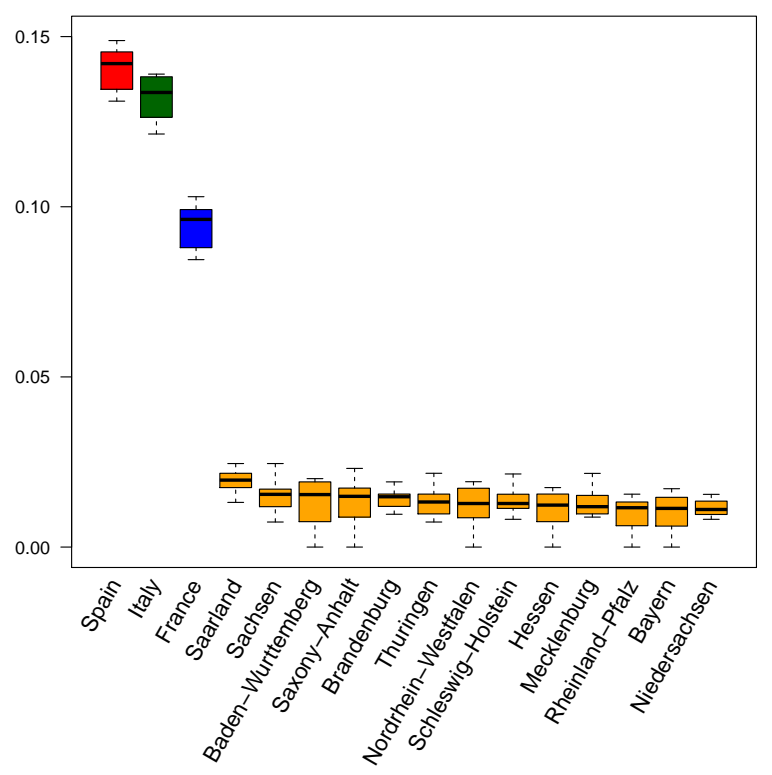

(c) Germany Regions and neighbours

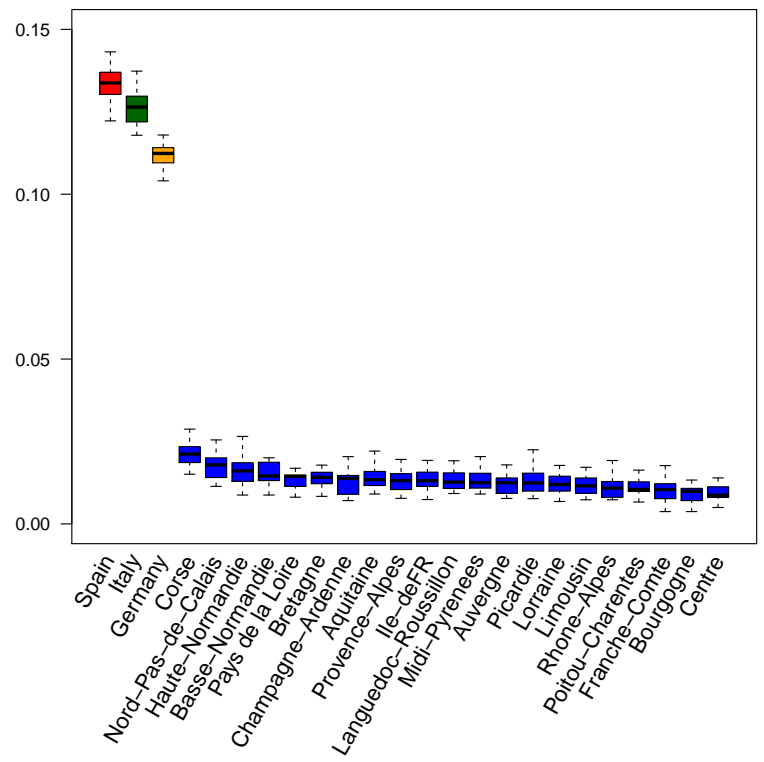

(b) France Regions and neighbours

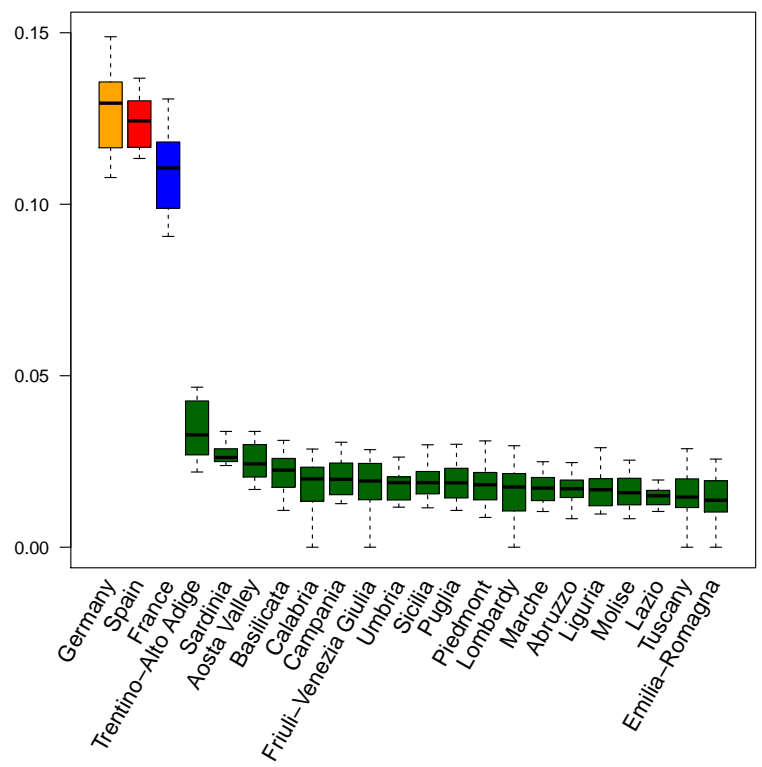

(d) Italy Regions and neighbours

Figure 4: Bottom-up measurement of subnational culture indicates substantial within-country cultural similarity. (A) subnational units in Spain are less distant from one another than from European cultural neighbors of Germany, Italy, or France. (B) French regions are culturally closer to one another than to European neighbors. (C) German regions demonstrate greater similarity to one another than to European neighbors. (D) Italian regions are culturally less distant to one another than to their European neighbors. subnational units in A-D are represented with boxplots that show the distance distribution between a given region and all other regions within the country under analysis. In the case of neighboring countries, boxplots show the distance distribution between that neighboring country and all the regions within the country under analysis.

Belgium and the Netherlands in the 1830s and the Partition of Ireland in the 1920s. Overall, this suggests the importance of national boundaries in shaping cultural distances. It also suggests that our measure captures deep cultural elements that persist over longer periods of time.

Do subnational cultures as measured via our Facebook data cluster together in a sensible manner? To ex- 


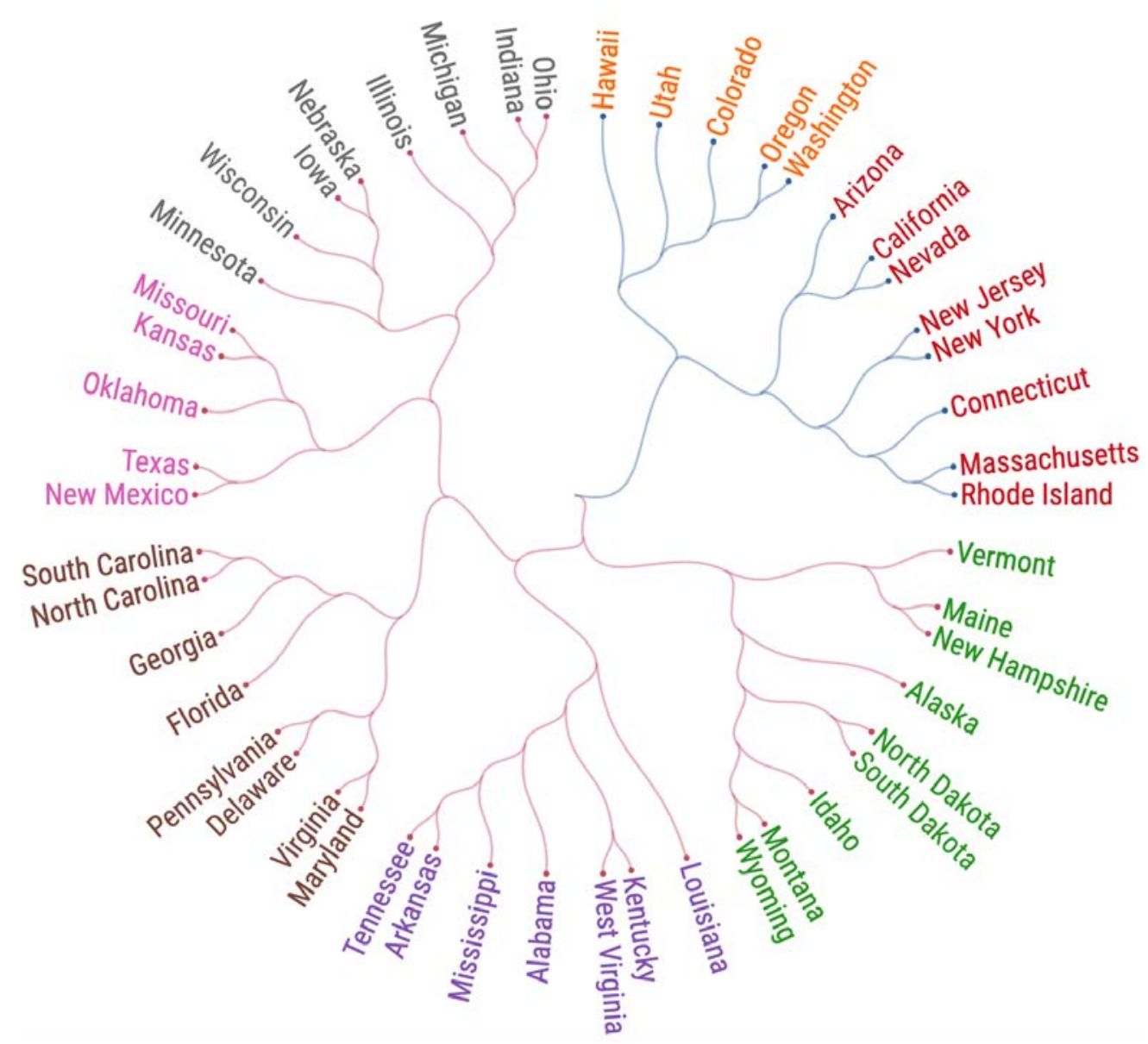

Figure 5: Bottom-up measurement of subnational culture returns sensible clustering of subnational units. Facebook culture vectors drawn from the states of the United States cluster together in an unsupervised manner into traditional regional and cultural groupings. South Carolina bears similarity to North Carolina, Montana to Wyoming, New Jersey to New York, and West Virginia to Kentucky. Similarities are not strictly geographic, with Alaska bearing similarity to New Hampshire and North Dakota, for example.

amine this question we calculate cosine distances for U.S. states and perform unsupervised clustering using the Ward linkage method (see Methods). Figure 5 presents the resulting dendrogram. The clusters return traditional regional and cultural groupings. For example, states in the U.S. Midwest are placed in proximity to one another, as are the states in the U.S. South. Interestingly, mountainous and more rural states also cluster together, with Alaska being closest to states like North Dakota, Idaho, and New Hampshire, despite the substantial geographic distances between them. This supports the idea that important cultural features are shaped by the physical environment ${ }^{67}$.

Figure 4 demonstrates that regions within countries can bear substantial similarity to one another. However, not all countries are likely have the same amount of within-nation cultural similarity. Do some countries have more regional cultural variation within them than do others? Figure 6 examines countries according to their interregional cultural divisiveness, or the average cultural distance between regions within a country (see Methods). Figure 6A ranks 18 selected countries in increasing order of interregional divisiveness. Two findings stand out in Figure 6A. First, developed countries exhibit smaller interregional divisiveness as compared to developing countries, suggesting they benefit from greater cohesiveness between regions. Second, within the 


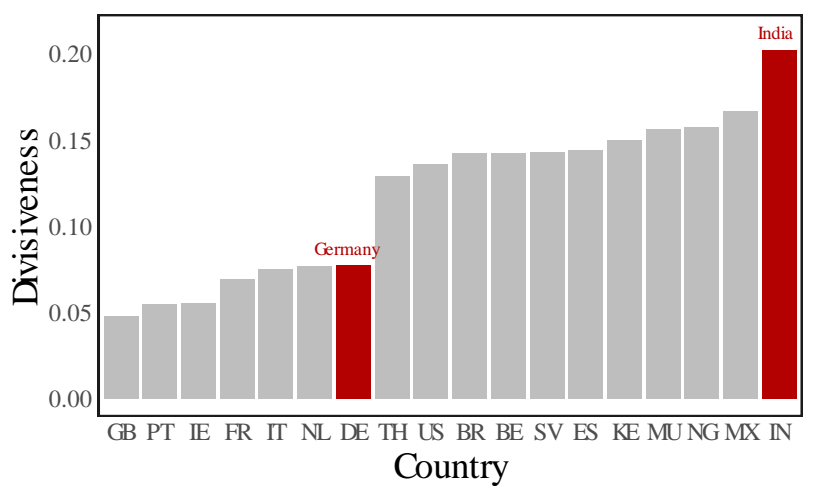

(a) Population-weighted regional divisiveness

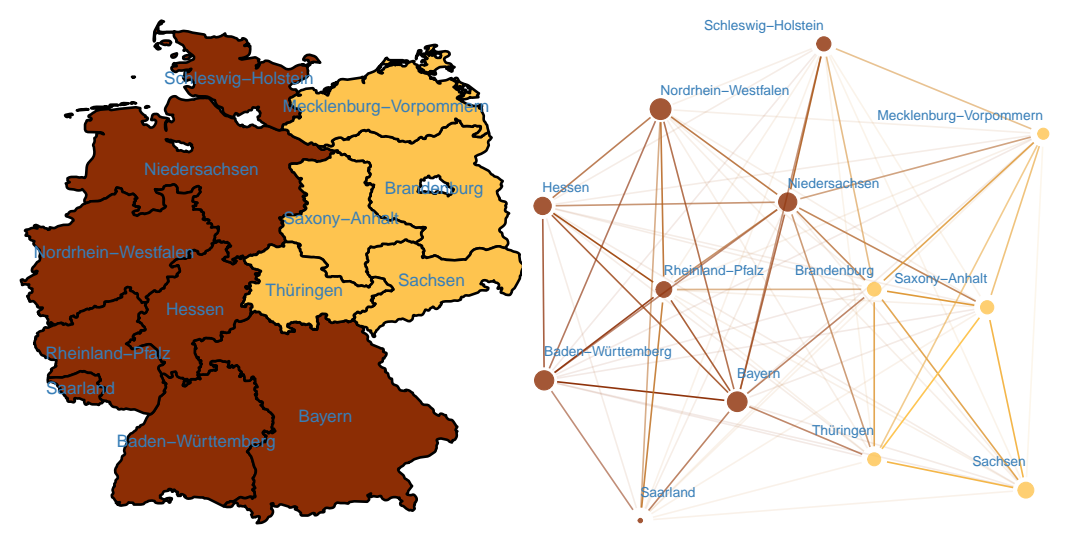

(b) Germany
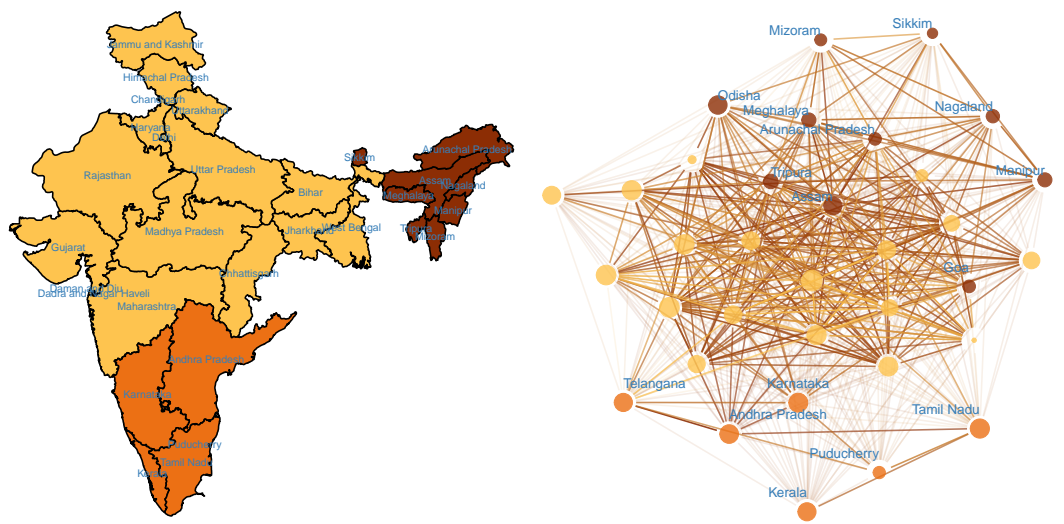

(c) India

Figure 6: Regional divisiveness. (A) Population-weighted regional divisiveness for 18 countries (range for number of regions: [3, 76]). Countries included are: BE: Belgium, BR: Brazil, DE: Germany, ES: Spain, FR: France, GB: Great Britain, IE: IN: India, Ireland, IT: Italy, KE: Kenya, MU: Mauritius, MX: Mexico, NG: Nigeria, NL: Netherlands, PT: Portugal, SV: El Salvador, TH: Thailand, US: United States. We chose these 18 countries to have a mix of developed countries and developing countries, as well as a mix of large and small countries. (B) Geographical map and network of regions in Germany (13 regions). Two communities of regions are detected. (C) Geographical map and network of regions in India (34 regions). Three communities of regions are detected. Networks are constructed from nodes as regions, and links are weighted by standardized cosine similarity between regions. Communities are detected using a multi-level modularity optimization algorithm (Louvain method) ${ }^{72}$. Nodes are resized proportionally to region population, and are colored according to community affiliation. Links are colored according to adjacent nodes, with lower transparency applied to higher weight links. Map regions are colored according to communities calculated from the corresponding network. 
group of developed countries, the three countries with the least interregional cohesiveness are Belgium, Spain and the United States. The first two have well-known regional issues, with threats of secession, whereas the third is a large geographic nation.

Figure 6B-C displays the geographical maps and networks of regions in Germany (13 regions) and regions in India (34 regions), respectively. We detect two communities of regions in Germany (see Methods), which map closely to the historical east-west divide in the country, suggesting this cultural divide still persists to this day. We detect three regional communities in India, which correspond roughly geographically with linguistic regions defined by the language families spoken within the country.

Figures 4, 5 and 6 highlight the utility of our measure in assessing subnational questions that are simply too expensive to measure with traditional quantitative approaches. Yet, differences in a society are not limited only to subnational differences. Societies can also differ along other identity cleavages, such as age, gender or race. Our measure enables us to also delve into the nature of cultural differences that vary according to such demographic groups and into differences that occur at even finer degrees of spatial resolution. Figures 7A and 7B explore whether countries that exhibit more divisiveness in one dimension also do so in other dimensions. It shows that age divisions and gender divisions have a weak positive association (Pearson correlation coefficient: 0.234 , p: 0.146), whereas countries that suffer from greater regional divisions have smaller differences between men and women. (Pearson correlation coefficient: -0.702, p: 0.001). Many developing countries exhibit more cohesiveness between genders, although they experience larger regional divides, compared to many developed countries.

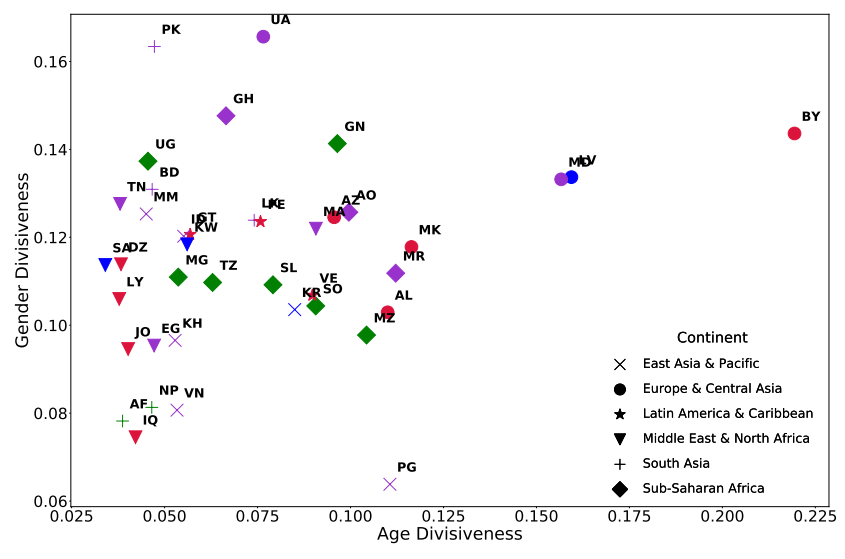

(a) Divisiveness by Age vs Gender

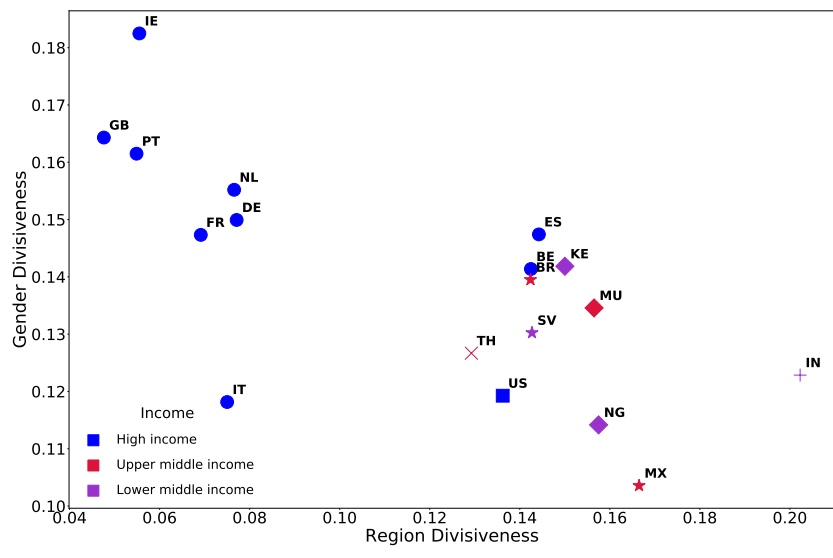

(b) Divisiveness by Regions vs Gender

Figure 7: Subnational age, gender, and regional cultural differences. Panel A shows a scatter plot of age and gender divisiveness for 40 countries. The gender divisiveness is computed as the cosine similarity between the FB penetration vector for women and men using our sample of Facebook interests. The age divisiveness is computed as the median cosine similarity between the Facebook culture vector of three different age groups 15-29, 30-64 and +65. (AF: Afghanistan, AL: Albania, AO: Angola, AZ: Azerbaijan, BD: Bangladesh, BY: Belarus, DZ: Algeria, EG: Egypt, GH: Ghana, GN: Guinea, GT: Guatemala, ID: Indonesia, IQ: Iraq, JO: Jordan, KH: Cambodia, KR: South Korea, KW: Kuwait, LK: Sri Lanka, LV: Latvia, LY: Libya, MA: Morocco, MD: Moldova, MG: Madagascar, MK: Macedonia, MM: Myanmar, MR: Mauritania, MZ: Mozambique, NP: Nepal, PE: Peru, PG: Papua New Guinea, PK: Pakistan, SA: Saudi Arabia, SL: Sierra Leone, SO: Somalia, TN: Tunisia, TZ: Tanzania, UA: Ukraine, UG: Uganda, VE: Venezuela, VN: Vietnam). Panel B shows a scatter plot of regional and gender divisiveness for 18 countries using (BE: Belgium, BR: Brazil, DE: Germany, ES: Spain, FR: France, GB: Great Britain, IE: Ireland, IN: India, IT: Italy, KE: Kenya, MU: Mauritius, MX: Mexico, NG: Nigeria, NL: Netherlands, PT: Portugal, SV: El Salvador, TH: Thailand, US: United States). 


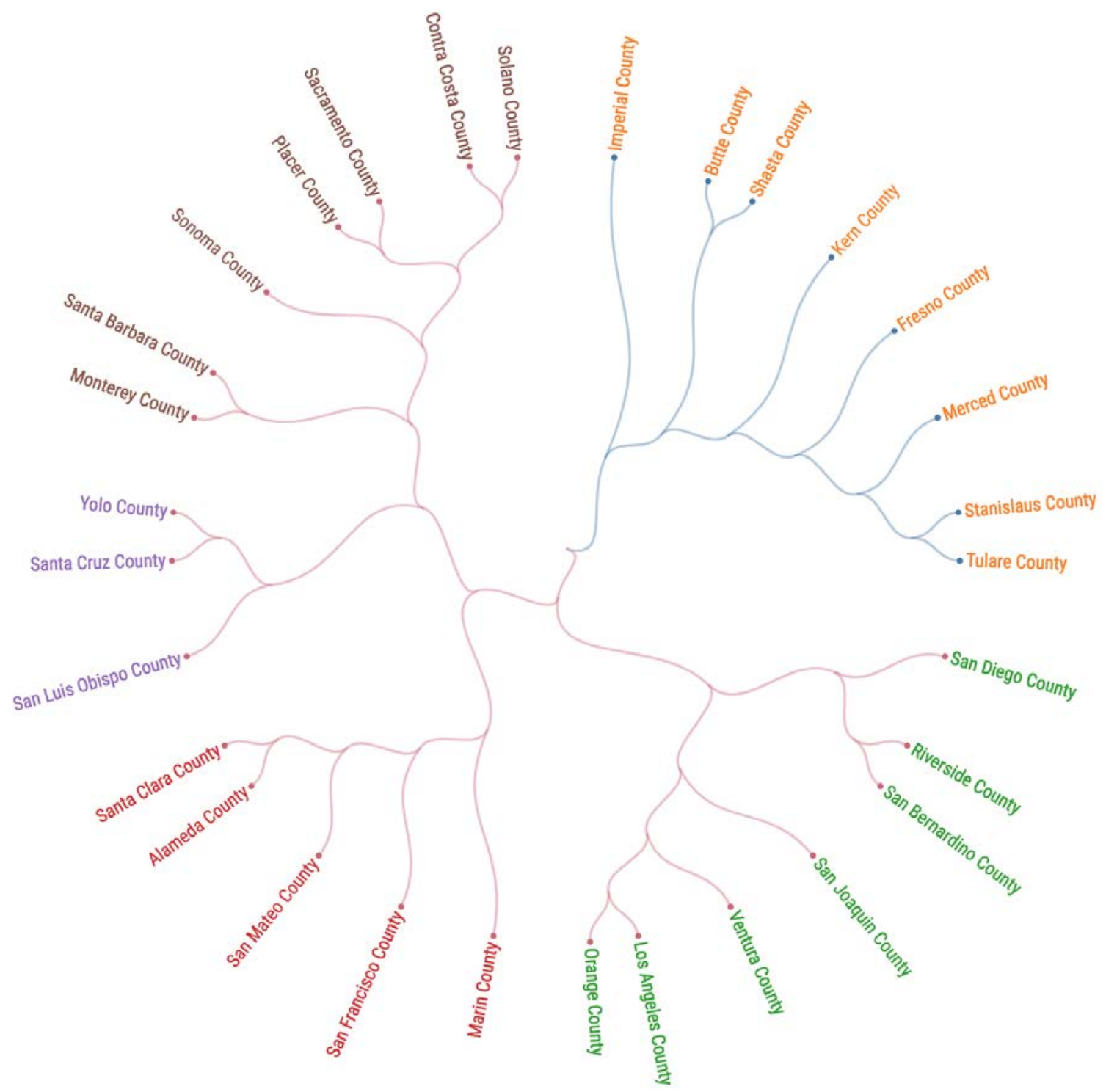

Figure 8: Local cultural similarities of California counties. Figure represents a dendrogram for California counties, based on cosine distances and Ward linkage method. In this figure we examine interests with at least 20,000 users in the state, and in counties with a population of more than 75,000 users. Our unsupervised clustering returns clusters that are culturally sensible. Los Angeles County is closest to Orange County, Tulare County is most similar to Stanislaus County, Santa Cruz County is proximal to San Luis Obispo County, and San Francisco County is nearest Marin County.

Further, our data enables us to investigate cultural similarities and differences at even higher spatial resolution. Figure 8 depicts the dendrogram of the cultural clustering of the most populous California counties. Geographically disparate but culturally similar counties - such as coastal surfing communities of San Luis Obispo and Santa Cruz as well as the rural inland counties of Imperial and Butte - are located next to one another in the dendrogram. Generalizing this approach would allow us to identify which local areas culturally diverge from the rest of the nation in which they are located, a phenomenon that might provide insight into regional political disaffection. 


\begin{tabular}{|c|c|}
\hline $\mathbf{A L}$ & $\begin{array}{l}\text { Frou Frou, United States Department of Commerce, GameZone, UAB Blazers men's basketball, Harvard Crimson football, } \\
\text { Indoor soccer, Traffic, Talladega Superspeedway, Hans Zimmer, Milo (drink), Shoal, Crow's feet, Nick Saban, University of } \\
\text { Alabama at Birmingham, Gulf Shores, Auburn Tigers, Auburn Tigers football, NCAA Football Division } 1 \text { championship, } \\
\text { Mobile, Auburn University, al.com, Alabama Crimson Tide, Alabama Crimson Tide football, University of Alabama }\end{array}$ \\
\hline $\mathbf{A K}$ & $\begin{array}{l}\text { Excursion, Maccabi Tel Aviv Basketball Club, Systems engineering, Shockwave (Transformers), Nissan H engine, Hospital } \\
\text { Corporation of America, Liquified Natural Gas,, Sinergia, Beauty Shop, Pyrotechnics, Ravex, Bryan Adams, Sustainable } \\
\text { development, Meal, Hydrographics (printing), Advanced Micro Devices, Magnetic field, Law and order (politics), Poor } \\
\text { posture, Palete (paint), Outlook.com, Small appliance, Sebastian, Bass Guitar, Baby, Paul Simon, Shareholder, Cephalopod } \\
\text { ink, Air freshener, Cervical vertebrae, True Colors (song), Sourdough, Popular Mechanics, Arctic Ocean, BP, Elopement } \\
\text { (marriage), Alaska Aces (PBA), Dividend, Lifejacket, Conglomerate (company), Gold Miner, Air force, Medical imaging, } \\
\text { Cigna, Homer, Valdez, Ocimum basilicum, Monopoly, African National Congress, Matsu Islands, Rangifer tarandus, Sled } \\
\text { dog, Jack White, Club, North Pole, Raven, Denali National Park and Preserve, Alaska Natives, GCI (company), Salmon, } \\
\text { Alaska Airlines, Arctic }\end{array}$ \\
\hline $\mathbf{A Z}$ & $\begin{array}{l}\text { Monsoon, Laser lighting display, Foreign language, Super Bowl XLVIII, Spritzer, Saguaro National Park, Super Bowl } \\
\text { XXX, Barrett-Jackson, Kodi, Super Bowl LI, John Madden, Steve Nash, Hot 98.3, American Physical Society, Anthony } \\
\text { Mackie, Karl Urban, Superstition, Arizona Coyotes, Surprise, The Phoenix (newspaper), Northern Arizona University, } \\
\text { sonoran desert, Larry Fitzgerald, Phoenix International Raceway, Arizona Diamondbacks, University of Arizona, Phoenix } \\
\text { Suns, State University of Arizona, FOX } 10 \text { Phoenix, ABC15 Arizona }\end{array}$ \\
\hline $\mathbf{A R}$ & $\begin{array}{l}\text { Catholic University Sports Club, Canis rufus, FINA, The Rev, asa, Eureka (U.S. TV series), John 3:16, Mallard, Mountain } \\
\text { Home, The Call ( } 2013 \text { film), National Education Association, White River (Arkansas), Eureka, Tom Cotton, Colton, Hi- } \\
\text { bachi, Channel } 7 \text { (Thailand), Sus scrofa domestica, N.W.A., Arkansas State University, Arkansas Razorbacks, University of } \\
\text { Arkansas }\end{array}$ \\
\hline CA & $\begin{array}{l}\text { University of California, San Diego Zoo Safari Park, California Academy of Sciences, Palm Desert, Univision } 34 \text { Los Ange- } \\
\text { les, Oakland Athletics, Algeria, Los Angeles Kings, California State Assembly, NBC Bay Area, Tea house, Staples Center, } \\
\text { Placer County, KRON-TV, San Jose Sharks, Six Flags Magic Mountain, Golden Gate Bridge, San Francisco Chronicle, Big } \\
\text { Bear Lake, KGO-TV, Monterey Bay Aquarium, City manager, ABC7 News, Gavin Newsom, La Jolla, Fox } 11 \text { Los Angeles, } \\
\text { KTVU, University of California Berkeley, California Lottery, Universal Studios Hollywood, KTLA, abc7, KABC-TV, Los } \\
\text { Angeles Times }\end{array}$ \\
\hline$\overline{\mathrm{CO}}$ & $\begin{array}{l}\text { Christian Social Union in Bavaria, Belly dance, Zeds Dead, Shire of Noosa, Check It Out! (Canadian TV series), The } \\
\text { Gazette (Montreal), Regenerative medicine, Polis, The Wild Animal Sanctuary, Blockbuster LLC, The Thin Blue Line } \\
\text { (emblem), Masterpiece (TV series), Miguel de Cervantes, New Belgium Brewing Company, Bad Influence (professional } \\
\text { wrestling), University of Denver, Bassnectar, Anschutz Entertainment Group, Energy supply, Pepsi Center, Rocky Moun- } \\
\text { tain National Park, Colorado Avalanche, University of Colorado Boulder, Denver International Airport, Denver Nuggets, } \\
\text { Colorado Rockies, Red Rocks Amphitheatre, KUSA (TV) }\end{array}$ \\
\hline CT & $\begin{array}{l}\text { Metro-North Railroad, Bomba (radio), Subsidy, First grade, Hebron, Chr } \\
\text { Heart University, Newsroom editorial, Dimension stone, Cava (Spanish win }\end{array}$ \\
\hline $\mathbf{D E}$ & $\begin{array}{l}\text { Mustela putorius furo, El Diablo (comic), Jack Russell terrier, Nemours, Apostolos Andreas, Half Baked, Crowdsourcing, } \\
\text { Joiner, Fire, Terracotta Army, Community gardening, Marine salvage, Roman legion, Free public transport, Betn, Oslo, } \\
\text { Women's National Basketball Association, Harvest (wine), Cold Case, Hammocks, Joe Biden, Leader (2010 film), The } \\
\text { News Journal }\end{array}$ \\
\hline FL & $\begin{array}{l}\text { Airline ticket, Enrique Santos Discepolo, Dunedin, Greyhound Race, Aventura Mall, Cuban cuisine, Bureau of Labor } \\
\text { Statistics, Miami Marlins, World Baseball Classic, The Pichy Boys, Pincho, Tampa International Airport, Jacksonville } \\
\text { Jaguars, Agustin of Hippo, Lake Worth, Fox } 35 \text { WOFL, WPLG, Downtown Disney (Walt Disney World), Tampa Bay Rays, } \\
\text { Disney's Hollywood Studios, WESH, Wikipedia, Orlando International Airport, Busch Gardens Tampa, Hialeah, WOFL, } \\
\text { Marco Rubio, Florida State Seminoles football, SeaWorld Orlando, WTSP, Tampa Bay Buccaneers, Natural Circus,, Space } \\
\text { Coast, Cruise line, Miami Dolphins, Epcot, Republican Party of Florida, University of South Florida, Hollywood, University } \\
\text { of Miami, Havana, ABC Action News - WFTS/TV, Florida Gators, Mysterious Client, Florida Gators football, University } \\
\text { of Central Florida, FOX13 \& myfoxtampabay.com, WFTS-TV, WFLA News Channel 8, WFTV, Florida State University, } \\
\text { Miami Beach, The Bahamas, Universal Studios Orlando, University of Florida, Cuba }\end{array}$ \\
\hline GA & $\begin{array}{l}\text { 5FM, Dress Up, Ray Charles, Angels Among Us Pet Rescue, Settler, Georgia Institute of Technology, Food court, Atlanta } \\
\text { Hawks, AJC, Atlanta Braves, 11Alive, Georgia Bulldogs football, Atlanta Falcons, Georgia Bulldogs, WAGA-TV, The } \\
\text { Atlanta Journal-Constitution }\end{array}$ \\
\hline HI & $\begin{array}{l}\text { Sashimi, Ginza, Macadamia, Philippine cuisine, YG Entertainment, Inertia, Windsurf, Palaos, Mick Fanning, Billabong, } \\
\text { American Wrestling Association, Red Bull Racing, Line Camera, Ryan Higa, Gheorghe Hagi, Kapuso Mo, Lexus NX, } \\
\text { Anne Curtis, Hokkaid, Economy class, Jollibee, Avex Group, Nepeta cataria, Toni Gonzaga, Bodyboarding, Nissan 350Z, } \\
\text { Iration, The Nice, Roots reggae, LINE, Rip Curl, Disney Tsum Tsum, Bruni, Sarah Geronimo, Driver, All Nippon Airways, } \\
\text { Blue Planet (role-playing game), Lion Dance, Kia Rio, Uplifting trance, TV Patrol, Kitesurfing, Yellowfin tuna, Professional } \\
\text { Association of Diving Instructors, Mai Tai, Kia Soul, Soldiers of Jah Army, Cebu, The Filipino Channel, Surfing Magazine, } \\
\text { Angel Locsin, Stain removal, Major Lazer, Kilusang Bagong Lipunan, Taro, Nanoparticle, Philippine Airlines, Hit and run, } \\
\text { Aloha shirt, Canterbury, Osaka, Itsjudytime, Pearl Harbour Attack, Damian Marley, Barangay }\end{array}$ \\
\hline
\end{tabular}




\begin{tabular}{|c|c|}
\hline ID & $\begin{array}{l}\text { mmy, Master formula, Sugar beet, Jim Rohn, Mendoza City, Black Swan (film), McCain Foods, Lip liner, Bengala, Rigby, } \\
\text { xiom, Organic Horticulture, Idaho Republican Party, Albin, Mosc, Melaleuca, Democratic Call, McCall, K12 (education), } \\
\text { concos, Idaho State University }\end{array}$ \\
\hline IL & $\begin{array}{l}\text { Uptown, Pilsen, History of the Chicago Bears, University of Illinois at Chicago, Luis Gutiérrez, Kankakee, Millennium } \\
\text { Park, Lollapalooza, Jimmy Butler, Goose Island Brewery, University of Chicago, Express mail, Chicago Transit Authority, } \\
\text { Chicago (band), Chicago Sun-Times, The Art Institute of Chicago, Allstate Arena, United Center, O'Hare International } \\
\text { Airport, Chicago Fire (TV series), Consumer protection, Canada Goose (clothing), Wrigley Field, Chicagoland Speed- } \\
\text { way, University of Illinois Urbana-Champaign, Portillo's Restaurants, Chicago Loop, Chicago White Sox, Chicago Police } \\
\text { Department, WFLD, WGN-TV, Chicago Tribune, Chicago Blackhawks, ABC } 7 \text { Chicago, Chicago Bulls }\end{array}$ \\
\hline IN & $\begin{array}{l}\text { john mellencamp, SpongeBob SquarePants (character), } 8 \text { Seconds, College soccer, Victor Oladipo, Network 18, Marketing } \\
\text { research, Notre Dame Fighting Irish, Butler University, Carmel-by-the-Sea, Indianapolis 500, Notre Dame Fighting Irish } \\
\text { football, Joe Donnelly, University of Notre Dame, Indiana Hoosiers men's basketball, Purdue University, Indiana Pacers, } \\
\text { Indianapolis Colts, WXIN, Indiana University }\end{array}$ \\
\hline IA & $\begin{array}{l}\text { Electrical oven, Australian rules football positions, Cash and carry, Conference hall, Diagnostic and Statistical Manual of } \\
\text { Mental Disorders, Chewable candy, Shawn Johnson, Rock Island (Illinois), Ashley Furniture Industries, Community school } \\
\text { (England and Wales), Iowa Hawkeyes, Iowa State University, University of Iowa }\end{array}$ \\
\hline $\mathbf{K S}$ & $\begin{array}{l}\text { Pillar, Massage chair, Martina McBride, Information and communications technology, Auditorium, Wichita State Shockers, } \\
\text { Helianthus, Kansas Speedway, Kansas Jayhawks, Kansas Jayhawks men's basketball, Kansas State University, Kansas City } \\
\text { Chiefs, Kansas (band) }\end{array}$ \\
\hline $\mathbf{K Y}$ & $\begin{array}{l}\text { Lakewood Church Central Campus, Sandalwood, Ashley Judd, Maker's Mark, Lutjanidae, Eden Hazard, House of Bourbon, } \\
\text { Bruce Willis, Folk music of England, Buffalo Trace Bourbon, Wildcat, Lazy river, Epinephelinae, Jim Beam, DeMarcus } \\
\text { Cousins, Woodford Reserve, Buffalo Trace Distillery, Medical assistant, Dental assistant, Mitch McConnell, Derby of } \\
\text { Kentucky, Felis silvestris, Bourbon Whiskey, Kentucky Wildcats, University of Kentucky }\end{array}$ \\
\hline LA & $\begin{array}{l}\text { Watts, Serbian Radical Party, Bourgeois , Concrete slab, Tubular bells, Pyhimys, Emeril Lagasse, Treme (TV series), Lour- } \\
\text { des, Society of Jesus, Aretha Franklin, Ante Christum Natum, Ulm, Zeus, West Bank, Nine Network, Swamp pop, Gonzales, } \\
\text { Basketball Hall of Fame, Hemline, French language, Iberia, Bowfishing, Street furniture, Tabasco, Float (parade), Minden, } \\
\text { Housing association, Roux, Orleans, Tabasco sauce, Jambalaya, Channel } 5 \text { (UK), Parish, Smoothie King, Lily flower, New } \\
\text { Orleans Jazz \& Heritage Festival, Raising Cane's Chicken Fingers, Daiquiri, Po' boy, United States Department of Home- } \\
\text { land Security, WTHI-TV, French Quarter New Orleans, New Orleans Pelicans, Carnival, Drew Brees, Cajun cuisine, lsu } \\
\text { tigers, King cake, Lake Charles, lsu, Bayou, Mardi Gras, Cajun }\end{array}$ \\
\hline ME & $\begin{array}{l}\text { Gramofon, Silly, MultiMediaCard, Swordfish, Per diem, Otto Waalkes, Mase, otto, Powiat, Turmaline, Ja Rule, Cover letter, } \\
\text { JCB (company), Vaccinium, Closer (2004 film), Spose, Dragonfly, Adult education, Synchronization, Paranormal Activity, } \\
\text { Freeport (Bahamas), Arlington National Cemetery, Haddock, Aura (parapsychology), Sustainable energy, Automated teller } \\
\text { machine, Patrick Dempsey, The Crown, Great Apple, Aura Dione, Park ranger, Savings Bank, Propane, Sugarloaf Mountain, } \\
\text { Bob Marley, Fishery exploitation, University of Maine }\end{array}$ \\
\hline MD & $\begin{array}{l}\text { Naval Academy, Egg white, Madonna fa } \\
\text { FF, Baltimore Orioles, The Baltimore Sur }\end{array}$ \\
\hline MA & $\begin{array}{l}\text { de Globo, Life annuity, Greater Sudbury, Primark, Harvard Medical School, Norma (technology), Berklee College of } \\
\text { usic, Commuter rail, Boston Marathon, Master of Fine Arts, National Lottery United Kingdom, University of Boston, } \\
\text { assachusetts Institute of Technology, WHDH (TV), Mass, The Boston Globe, WCVB Channel } 5 \text { Boston, WCVB-TV }\end{array}$ \\
\hline MI & $\begin{array}{l}\text { Kontinental Hockey League, Rosa Parks, Miguel Cabrera, Sparty, Machine shop, Grand Hotel (Mackinac Island), United } \\
\text { Automobile Workers, Pontiac, Water polo, Michigan International Speedway, Chuck, North American International Auto } \\
\text { Show, Michigan Wolverines, Gant, TV8 (Turkey), Nassar, Henry Ford, Michigan State Spartans football, Novi, Michigan } \\
\text { Wolverines men's basketball, Kalamazoo, Michigan State Spartans, WDIV-TV, Lansing, Detroit Free Press, WXYZ-TV, } \\
\text { Detroit Pistons, Detroit Red Wings, Michigan Wolverines football, Detroit Lions, Meijer, University of Michigan, Michigan } \\
\text { State University, Detroit Tigers, Pure Michigan, Great Lakes }\end{array}$ \\
\hline $\mathbf{M N}$ & $\begin{array}{l}\text { ius, Purple Rain (album), Punter (football), Bauhaus, Martin Luther, Hells kitchen, Red Digital Cinema Camera Com- } \\
\text { ny, Caucus, Skol, Current TV, Target Center, First Avenue (nightclub), Minneapolis Saint Paul International Airport, } \\
\text { innesota Golden Gophers football, Fortification, Al Franken, Minnetonka, Minnesota Timberwolves, KMSP-TV, KARE, } \\
\text { iversity of Minnesota }\end{array}$ \\
\hline MS & $\begin{array}{l}\text { Australian Defence Force, Merit (Buddhism), Saint Dominic, Free area of the Republic of China, Kicker (sports magazine), } \\
\text { Bertrand Russell, Skybox (video games), Juju (singer), Nationalist Movement Party, Acadiana, Big K.R.I.T., Pier (architec- } \\
\text { ture), Dionysus, anna, Second base, Treasure hunting, Cowbell (instrument), Casabella, Wicker, Hideaway, University of } \\
\text { Mississippi, Mississippi State University }\end{array}$ \\
\hline MO & Hannibal (TV series), Drury University, St. Peter's Basilica, George Sol \\
\hline MT & $\begin{array}{l}\text { Wine and food matching, The Jam, Bracket (tournament), Lucca, Escapology, Buddhahood, Kroger 250, Crocus sativus, } \\
\text { Bill Pullman, Twisted Sister, Calgary, Talib Kweli, Goldsmith, Gareth Bale, Pasty, Crow Nation, Onsen, Mission Valley, } \\
\text { Mint.com, Alberta, Mike Will Made It, Top hat, Lewis and Clark Expedition, Grizzly bear, Glacier National Park, Cross } \\
\text { country running, Great Falls, Big Sky Conference }\end{array}$ \\
\hline
\end{tabular}




\begin{tabular}{|c|c|}
\hline $\mathbf{N E}$ & $\begin{array}{l}\text { nporium, Vega, Oneworld, Clock tower, Jake Olson Studios, Pork steak, Spareribs, Kolache, Strip steak, Take-Two Inter- } \\
\text { tive, UANL Tigers, Pinewood Studios, Old Market (Omaha, The Good Life (band)), Nippon Television, Airport lounge, } \\
\text { ornell University, Scooter (band), Abraham Lincoln, WOWT, University of Nebraska-Lincoln }\end{array}$ \\
\hline$\overline{\mathbf{N V}}$ & $\begin{array}{l}\text { Glazier, Pereira, Los Angeles Sparks, Sportsbook, Just Do It, Green Man, Mexico's beers, Tao, Country blues, Paris Las } \\
\text { Vegas, William Hill (bets), Law empire, Mac Mini, Rick Harrison, Gene Simmons, MGM Grand Garden Arena, Sin City } \\
\text { (film), Circus Circus Las Vegas, Dean Martin, Absinthe, Panasonic Corp, List of mayors of Las Vegas, Sahara Desert, Planet } \\
\text { Hollywood Resort and Casino, Hakkasan, Palace, LVH Las Vegas Hotel \& Casino, IMac, Truckee, The Mirage, Palms } \\
\text { Casino Resort, ARIA Resort \& Casino, Nen, Cineplex Entertainment, Sierra Nevada (U.S.), Ol' Dirty Bastard, Caesars } \\
\text { Palace, Flamingo Las Vegas, Las Vegas Motor Speedway, MacBook, Encore Las Vegas, Bellagio (resort), Mandalay Bay, } \\
\text { Wynn Las Vegas, Sparks (Nevada), Fremont Street Experience, MGM Grand Las Vegas, The Venetian Las Vegas, Lago } \\
\text { Tahoe, Las Vegas Strip, Judi }\end{array}$ \\
\hline NH & $\begin{array}{l}\text { cus, SIG Sauer P239, LaBelle, Social studies, Millennium, Keno, White Mountain, Market basket, Treasurer, Weighing } \\
\text { ale, Alfred Hitchcock }\end{array}$ \\
\hline NJ & $\begin{array}{l}\text { Holiday Magic, Narendra Modi, Caesars Atlantic City, Open Rights Group, Epic Vines, Empanada, Tropicana Casino \& } \\
\text { Resort Atlantic City, New Jersey Devils, Bruce Springsteen, Six Flags Great Adventure, Rutgers University, ShopRite } \\
\text { (United States), Jersey Shore }\end{array}$ \\
\hline$\overline{\text { NM }}$ & $\begin{array}{l}\text { Concerto, Jinja (Uganda), Cuauhtemoc, Makeup Artist, Opel Mokka, Chihuahua, Montreal Expos, Roadrunner Records, } \\
\text { Apodaca, Lil Rob, Artist collective, Horse harness, Palette, Saddle blanket, Río Grande, Benito Juarez, Forest, Grupo of } \\
\text { 15, Triple J, Better Call Saul, Baby Bash, Los Alamos National Lab, New Mexican cuisine, Launchpad, Daily Times (Pak- } \\
\text { istan), Gallup survey, Blood In Blood Out, Adobe, Wyndham, Martinez, New Spain, Navajo Nation, Argentina provinces, } \\
\text { Independiente Santa Fe (soccer), Sandia National Laboratories, Holly Holm, Chile }\end{array}$ \\
\hline NY & $\begin{array}{l}\text { Guyana, Chelsea (Manhattan), Lower East Side, Moovit, New York Major, Tribeca, Upper East Side, Renminbi, Buffalo } \\
\text { Sabres, Syracuse University, New York University, Newsday, State University of New York, The Citizen, History of the } \\
\text { Buffalo Bills, Emergency status, Juno }\end{array}$ \\
\hline $\mathbf{N C}$ & $\begin{array}{l}\text { Davidson College, The Orange Peel, Bastion, DeAngelo Williams, SouthPark (Charlotte neighborhood), Carolina Hurri- } \\
\text { canes, Charlotte Motor Speedway, Wake Forest University, Charlotte Hornets, FOX8, Duke University, North Carolina Tar } \\
\text { Heels, WSOC-TV, North Carolina Tar Heels men's basketball, Research Triangle, University of North Carolina at Chapel } \\
\text { Hill }\end{array}$ \\
\hline ND & $\begin{array}{l}\text { Fargo (TV series), Minotaur, Sloppy Joe, Radiating Floor, Iron Cross (band), Miniature horse, Toronto Blue Jays, Airbrush } \\
\text { makeup, Bully ( } 2011 \text { film), Sweet onion, Cherry tomato, Environmental protection, Blow, Kiwifruit, Cutter (wrestling), } \\
\text { Diamond cutting, Desmonte, Antonn Dvok, Next plc, Bowling (cricket), Verizon VIP Tour, Hanson, Kudos (production } \\
\text { company), Noodle Soup, Warehouse club, Vendor, Asparagus, The Big One (NASCAR), Winnipeg, Tutti frutti (food), } \\
\text { Fergie, Canadian Pacific Railway, Human resources, Railroad classes, Teppanyaki, Roadhouse (facility), Board certification, } \\
\text { Contemporary hit radio, Rooster, Food cooperative, Manitoba, Thermostat, Stove (heater), Josh Duhamel }\end{array}$ \\
\hline$\overline{\mathbf{O H}}$ & $\begin{array}{l}\text { limanjaro, Epiphone, JR Smith, } 3 \text { Austria Top 40, Biodiesel, Kettering, Lebron James 23, Mor } \\
\text { spital, Developmental disability awareness, Kevin Love, University of Ohio, Cleveland Cavalier } \\
\text { cial movements, Ohio State Buckeyes men's basketball, The Plain Dealer, Cleveland Browns, Buck } \\
\text { valiers season, Cleveland Indians, Lake Erie, WJW (TV), Ohio State Buckeyes, Cleveland Cavalier } \\
\text { tball, Ohio State University }\end{array}$ \\
\hline OK & $\begin{array}{l}\text { ea, Austin Metro, Cushing, WFXT, Television South, Twister, Rhema, Enes Kanter, Corporate identity, Altus, Texas } \\
\text { rn, Dren, Oklahoma State Cowboys baseball, Aeroelasticity, Fallin, Serge Ibaka, Hollyoaks, OKC Thunder on } \\
\text { rts, Sharpe (TV series), Velociraptor, Kevin Durant, Petting zoo, Outlaw country, Stillwater, Cuff, Red Dirt (music), } \\
\text { Westbrook, Oklahoma Sooners, Oklahoma State UniversityStillwater, Oklahoma Sooners football, University of } \\
\text { ma }\end{array}$ \\
\hline OR & $\begin{array}{l}\text { Sutherland, Seaside, Clog, Food trucks, Wilco, Portlandia (TV series), Damian Lillard, Le Figaro, Portland Timbers, } \\
\text { Brown, Moda Center, Oregon State University, Oregon Ducks football, Willamette River, Portland Trail Blazers, } \\
\text { V, University of Oregon, Portland International Airport }\end{array}$ \\
\hline $\mathbf{P A}$ & $\begin{array}{l}\text { Pittsburgh Panthers, Pittsburgh Panthers football, Universit } \\
\text {, Pennsylvania State University }\end{array}$ \\
\hline RI & $\begin{array}{l}\text { arranquilla, Cable car, Teatro Colón, Awakenings, Venda, Immersion (virtual reality), Anti-aircraft warfare, Supernova, } \\
\text { ologna, Virtual Private Network, Austrian Hockey League, Tilly's, Siena, Colosseum, Prosecco, Kids club, Swansea, } \\
\text { epartment of Health (United Kingdom), The Andes, Providence Bruins, Edible Arrangements, Tulip, Strand, Paul van } \\
\text { yk, Brown University, Oyster bar, NBC10 Philadelphia, WJAR }\end{array}$ \\
\hline SC & $\begin{array}{l}\text { Sem, Solicitor, Darlington Raceway, Windows Vista, Okra, The Citadel, Car Town, Fencing, WACH, Dropkick Murphys, } \\
\text { Home appliance, Moon, Clemson Tigers, Clemson Tigers football, South Carolina Gamecocks, University of Columbia, } \\
\text { Clemson University, University of South Carolina }\end{array}$ \\
\hline SD & $\begin{array}{l}\text { ographic information system, Crazy Horse, Hitch, Sun News (India), Broasting, Chipmunk, Rosebud, Cantons of } \\
\text { vitzerland, Food marketing, Karisma Kapoor, Personal Integrity, Say Anything, Milky Way, Giovanni Sio, Maxwell } \\
\text { ccer player), Box Lacrosse, Jonas Bjrkman, Hypnosis, Pepper, Pheasant, Agricultural show, San Diego State University, } \\
\text { orm chasing, Lakota people, Mount Rushmore, Kota, Black Hills }\end{array}$ \\
\hline
\end{tabular}




\begin{tabular}{|c|c|}
\hline TN & $\begin{array}{l}\text { Samsung Galaxy Note II, Google Nexus, Samsung Galaxy Note 3, Johann Heinrich Friedrich Link, Samsung Galaxy Note } \\
\text { 4, Samsung Galaxy S4, Samsung Galaxy S5, Samsung Galaxy S6, Samsung Galaxy S III, IPad 2, Bodyguard, Populus, } \\
\text { Mobile Bank, Seedling, Bonnaroo, TriStar Pictures, Brentwood, Dollywood, Memphis Grizzlies, Vanderbilt University, } \\
\text { History of the Tennessee Titans, Tennessee Titans, Network Ten, Tennessee Volunteers football, University of Tennessee }\end{array}$ \\
\hline TX & $\begin{array}{l}\text { Alicia Villarreal, Telemundo Houston, Election day, Monterrey Football Club, Cantera (soccer), Chow Chow, Jeweler, San } \\
\text { Antonio Spurs on 247Sports, Lamar University, Texas Longhorns men's basketball, Central Market (Texas), UK Inde- } \\
\text { pendence Party (UKIP), Texas A\&M Aggies, The Island (2005 film), Texas A\&M Aggies football, South by Southwest, } \\
\text { Tamaulipas, Baylor University, San Antonio RiverWalk, Quest, University of Houston, Bimbo Group, Center, South Padre } \\
\text { Island, Texas Tech University, Texas State University, Local H, Alamo Mission in San Antonio, University of North Texas, } \\
\text { University of Texas System, The Dallas Morning News, Texas Longhorns, Texas Longhorns football, La Mafia, WFAA, } \\
\text { Ram Gopal Varma, FOX } 26 \text { Houston, Sugar Land, Gallery Furniture, Houston Livestock Show and Rodeo, Houston As- } \\
\text { tros, Houston Chronicle, FOX } 4 \text { News, Texas Parks and Wildlife Department, KHOU, 5miles, KTRK-TV, Houston Texans, } \\
\text { Texas Hill Country, San Antonio Spurs, Greg Abbott, University of Texas at Austin, Dallas-Fort Worth International Airport, } \\
\text { Whataburger, H-E-B }\end{array}$ \\
\hline UT & $\begin{array}{l}\text { Deron Williams, Amanda Miguel, Short Selling, Good Charlotte, La La Land (film), Holi, Task force, Elektra Records, } \\
\text { Mission (LDS Church), Gordon Hayward, Hyundai Accent, Fairy godmother, Star Trek II: The Wrath of Khan, Zermatt, } \\
\text { Forecasting, Stake, Donny Osmond, Transformers (toy line), Leandro \& Leonardo, Jordan river, Elevate, Temple (SUD), } \\
\text { Homie, Ward (LDS Church), Drainage, Roy, Cotopaxi, Draper, Zion National Park, Best Friends Animal Society, David } \\
\text { Archuleta, Scottish League Cup, Price, This Morning (TV programme), The Piano Guys, Temple (SUD), Real Salt Lake, } \\
\text { FamilySearch, Mitt Romney, Southland Conference, Mormon Channel, Cafe Rio, Book of Mormon, Joseph Smith, Brigham } \\
\text { Young University, Utah Jazz, Mormon, University of Utah, The Church of Jesus Christ of Latter-day Saints in Hawaii, } \\
\text { History of The Church of Jesus Christ of Latter-day Saints, Latter Day Saint movement, KSL-TV }\end{array}$ \\
\hline VT & $\begin{array}{l}\text { Cray Inc., ECCO, Australia and New Zealand Banking Group, Shopping bag, George Orwell, Fancy-Dress Party, Carina, } \\
\text { 350.org, Political movement, Bureau of Alcohol, Berna, Shawarma, The Wood, Mountain Equipment Co-op, Ethan Allen } \\
\text { (furniture company), City Market (US grocery store chain), Compost, Eye (cyclone), Charcuterie, Prohibition in the United } \\
\text { States, Water supply, New York City Police Department, The Alchemist (novel), Phish, BTV (Bulgaria), RT (TV net- } \\
\text { work), Alderman, Montreal, Seventh Generation, Burton Snowboards, Lockheed Martin F-35 Lightning II, Hilton Hotels, } \\
\text { Irish pub, The Nature Conservancy, Green Mountain, King Arthur Flour, Sheraton Hotels and Resorts, Oktoberfest, Local } \\
\text { consumption, Ben \& Jerry's, Cider, Maple syrup, U.S. Senator Bernie Sanders, University of Vermont }\end{array}$ \\
\hline $\mathbf{V A}$ & $\begin{array}{l}\text { Apple cider, George Mason University, Squash, The National, Tim Kaine, Rayo Vallecano, University of Virginia, Virginia } \\
\text { Polytechnic Institute and State University, Newport News, Virginia is for Lovers }\end{array}$ \\
\hline WA & $\begin{array}{l}\text { Swan Lake, Vancouver Island, Habanero, Container garden, Mini-bar, Bill and Melinda Gates Foundation, Noise pollu- } \\
\text { tion, Whistler, Epicureanism, The News Tribune, The Stranger, Melinda Gates, Millwork (building material), Washington } \\
\text { Huskies football, Road Violence, Macklemore, Evergreen, 12th man (football), Washington Huskies, Pete Carroll, Wash- } \\
\text { ington State University, Pike Place Market, Seattle Sounders FC, Rowing, Seattle Seahawks on 247Sports, Marshawn } \\
\text { Lynch, Mount Rainier, Seattle Mariners, University of Washington, Richard Sherman, KCPQ, Richard Sherman (american } \\
\text { football), KING-TV, KIRO } 7 \text { News, Russell Wilson, KIRO-TV, Puget Sound, The Seattle Times }\end{array}$ \\
\hline WV & $\begin{array}{l}\text { Reproductive rights, ABP News, Clutch, SuperCity, Andrew McCutchen, Naloxona, Boxing ring, Snuff (tobacco), Canaan, } \\
\text { Delegate, Civil service, Mitsubishi Outlander, Modem, The Ring, Metroxpress, Jennifer Garner, Telegram, County commis- } \\
\text { sion, Marshall Thundering Herd football, WCW Monday Nitro, Pepperoni, Political geography, Big Buck's, Coal mining, } \\
\text { Marshall University, Brad Paisley, Appalachian, Hiking, West Virginia University }\end{array}$ \\
\hline WI & $\begin{array}{l}\text { Dizzy Gillespie, Road America, Gastroenterology, Milwaukee Admirals, Giannis Antetokounmpo, Bay View, WAXX, Mad } \\
\text { City (film), Carl Maria von Weber, Miller Brewing Company, Lake Geneva, Orange Bowl, Marquette University, Fish fry, } \\
\text { Charles Woodson, Pabst Blue Ribbon, The Rave / Eagles Club, Eddie Lacy, Clay Matthews III, Brett Favre, Summerfest, } \\
\text { Supper club, Pro Bowl, Green Bay Packers on 247Sports, Wisconsin Badgers football, Wisconsin Badgers men's basketball, } \\
\text { Wisconsin Badgers, University of Wisconsin-Madison, Green Bay Packers }\end{array}$ \\
\hline WY & $\begin{array}{l}\text { Chapters, Table Mountain, Maternal bond, Quill, Sore (band), Excalibur (film), Benchmarking, Sapporo, Public service, } \\
\text { Polydor Records, Garter (stockings), Casper the Friendly Ghost, Bring It On, All That Remains, Green River (band), Cold } \\
\text { Stone Creamery, Ribs (food), Grand Teton National Park, Gillette (brand), Cod, Air medical services, Fremont (California), } \\
\text { Cartridge (firearms), Medical emergency, Mountain range, Casper (film), Fish and chips, Mountain West Conference, Diving }\end{array}$ \\
\hline
\end{tabular}

Table 1: Cultural outliers for U.S. states. This table shows for each US state the cultural outliers computed as an interest that presents a penetration in that state that is at least twice higher than that interest's share in any other U.S. state. Any interest referring to a geographical city, town, or county located within the state under analysis has been filtered from the list of cultural outliers.

Our method also enables us to answer an additional fundamental question regarding human culture: Which cultural attributes make a nation, region, or locality unique? Table 1 presents the cultural outliers in our data for the 50 U.S. states, where a cultural outlier is defined as an interest that presents a penetration in that state that is at least twice higher than that interest's share in any other U.S. state (see SI: Retrieving the cultural 
outliers of US regions). Cultural outliers include sports teams, television shows, and cuisines particular to a state that are likely relatively common knowledge among U.S. residents. However, the cultural outliers also include information that would be very difficult and costly to traditionally quantify: New Hampshire has the highest penetration of Alfred Hitchcock fans, Delaware has the highest penetration of interest in the Jack Russell terrier, and Montana has the highest rate of interest in the Lewis and Clark Expedition, among hundreds of other previously unobservable cultural outlier interests.

Our method lays out a complementary approach to the study of culture that is increasingly able to be measured via the application of computational social science to the ever larger portion of human lives that are unobtrusively and observationally measured online and offline. Doing so with our sample of Facebook interests for countries and subnational and local regions around the world indicates that our bottom-up measure of culture corresponds positively to traditional quantitative measures, contains a greater amount of explanatory dimensions, enables the clustering of countries, subnational units, and localities into sensible groupings, and provides insight into cultural variation at unprecedented spatial, demographic, and topic-based resolution. While our approach here expands dramatically on traditional methods and uncovers novel insights into cultural variation, a further strength of our approach is its ability to enable answering questions about human culture that have been - up to this point - impossible to investigate at scale using traditional quantitative methods. For example, our method and data can enable investigating questions such as: 'Which country is the cultural center of the world?' (see SI: Cultural center of the world) and 'Which is the global 'sister region' of a particular region within a country?' (see SI: Cultural sister regions).

\section{Discussion}

The high spatial (zip code-level) and temporal (daily) resolution of Facebook's available data, coupled with the more than 200 million individuals on the platform in the United States and the more than two billion on the platform around the world, enable measuring cultural differences with remarkable precision. Computing cultural differences between subnational regions (Figures 4, 5 and 6), cities, counties (Figure 8), or any different subgroups of any country (Figure 7), cost prohibitive when using traditional surveys, now becomes a straightforward endeavor. These studies are possible to conduct freely via the publicly available data provided by the Facebook Marketing API. Even so, this API is limited relative to what is theoretically possible, given the magnitude of human behavioral data that firms are currently collecting globally.

While we believe our conception and measurement of culture provides numerous complementary benefits to traditional measures, a number of considerations are worth noting. For one, that our approach does not inductively distill culture into parsimonious concepts means that the constellations of interests and behaviors that might diverge between two cultures may not always lend themselves to ease of conceptual interpretation. A few of the cultural outliers in Table 1 highlight this consideration.

Further, while our Facebook measure of culture represents a marked improvement in terms of its ability to measure the surface of culture as compared to traditional surveys with high rates of non-response and relatively few questions ${ }^{52}$, it is still far from perfect. Not all individuals in every country around the world are on Facebook. Our an alysis only generalizes to differences among those that use Facebook (however, our validity results persist even when looking at countries with lower penetration of Facebook use, see SI: Robustness to 
alternative samples). Additionally, while nearly 60,000 dimensions represent a dramatic increase over traditional top-down methods (of three orders of magnitude, see Figure 2A), they still measure only a fraction of the full dimensionality of culture. Moreover, these dimensions themselves suffer from a certain endogeneity of measurement: Facebook does not have an interest listed for every possible feature of culture. The ones it chooses to classify are endogenous to the platform itself. For example, these interests exclude certain topics - like sex and hate speech - banned from the platform. A more ideal computational system would classify all interests at an even finer scale of demarcation.

Finally, while we believe our measure possesses numerous complementary benefits to traditional quantitative and qualitative approaches to culture - unobtrusiveness, scale, resolution, richness and breadth of constructs, and the ability to freely peer into the lives of billions of people - our measure emphatically cannot substitute for traditionally employed approaches to culture ${ }^{73}$. If we observe that individuals in a place spend substantial time looking at religious websites, we cannot know to what degree they personally hold religion as important: traditional approaches are needed to investigate further. And while our method is useful in providing a culturomic $^{74,75}$ barcoding $^{76,77}$ of global culture, the classification and interpretation of culturally important factors still necessitates in depth and careful study of features uncovered by our approach - as well as of those features of culture our measure omits.

Humans around the world share many cultural similarities but also have many differences. Until very recently, quantitatively measuring vast portions of culture was impossible. However, as we collect data on humanity it becomes increasingly possible to measure the surface of human culture in manners that approach cultures' full underlying dimensionality. Doing so - in addition to furthering traditional quantitative and qualitative approaches to culture - will aid in a richer understanding of global human culture.

This improved ability to measure cultural differences between population groups at a more granular level enables a dramatic advance in the evaluation of some of the most pressing questions in the social sciences, such as: Do national borders shape cultures? Are societies more likely to fracture along gender lines, racial lines, or regional lines? Which specific locations on the globe are more prone to civil conflict and violence? Does a lack of cultural cohesiveness contribute to political extremism? Are certain immigrant cultures more adept at integrating than others? Some of these questions have been touched upon in this paper; others have not. All have one element in common: answering them requires cultural measurement at previously unavailable scales and resolutions.

\section{Acknowledgements}

A.C. acknowledges funding from the European Union's Horizon 2020 innovation action program under grant agreement No 786741 (SMOOTH project); and the Ministerio de Economía, Industria y Competitividad, Spain, and the European Social Fund (EU), under the Ramón y Cajal program (Grant RyC-2015-17732). R.C. acknowledges funding from H2020 EU Project PIMCITY (Grant 871370 ) and the Taptap Digital-UC3M Chair in Advanced AI and Data Science applied to Advertising and Marketing. I.O. acknowledges funding from ECO2013-42710-P, MDM 2014-0431 and Fundacion BBVA. I.M. acknowledges funding from Spanish Ministry of education with the FPU programme (FPU15/03518). The authors thank Niccolo Pescetelli and Alex Rutherford for their helpful comments. 


\section{Methods}

This section starts by providing details on the process of collecting Facebook interests and on the calculation of Facebook distances. We then discuss the data sources for our other distance measures (genetic, linguistic, geographic, religious, WVS). In addition, we explain the methodology for creating dendrograms, for conducting principal component analysis, and for calculating and analyzing our regional divisiveness.

\section{Facebook Marketing API}

We collect data on nearly 60,000 Facebook interests across countries and territories, European subnational regions, U.S. states, California counties, and various demographic subgroups between 2017 and 2018 using Facebook's Marketing API (see https://developers. facebook.com/docs/marketing-apis, last accessed: April 2019). Note that for some experiments, such as the gender-region analysis or the US counties analysis, we used a subset of interests, since some interests in the original set did not have enough users when considering smaller user groups. This collection process yields a vector of the number of Facebook users in each entity that holds each interest, which in turn serves to create a vector containing entity-interest shares. Using this vector of interest shares, we compute distances between each group of interest.

Advertisers configure their ad campaigns on Facebook through Facebook Ads Manager which can be accessed through a dashboard that queries the Facebook Marketing API. This interface, which is also accessible to the public, allows advertisers to define the group they want to target with their advertising campaigns, i.e., the population of interest. The group specifications can include geographic location (country, region, city, zip code, latitude/longitude...), demographics (gender, age, language, education,...), behaviors (mobile device, operating system, browser,...), and interests (sports, food, cars, art,...). For a given set of group specifications, the Facebook Marketing API provides the number of monthly active users (MAU), daily active users (DAU), and different advertising costs (per click, per thousand visualizations, etc.).

For our analysis, we gather information on the number of users in each group who hold different interests. It is therefore important to clarify how Facebook assigns interests to individuals. A user is identified as having a particular interest based on their data and activity on Facebook, as well as on external websites, apps, and online services where Facebook has a presence. (Facebook has been estimated to have a presence on over $30 \%$ of popular websites $)^{78}$. As individuals spend an increasing share of their time in the proximity of devices that track their location, Facebook also knows many dimensions of its users' offline activity, such as whether they go to football games, spend times downtown, or attend religious services.

Once a user is identified as being interested in "cars", she will be included as a potential target of any advertising campaign configured to reach users interested in cars. There are hundreds of thousands of interests, spanning huge swaths of human preferences. To give an idea of the breadth and comprehensiveness, Facebook organizes interests in a multi-level hierarchical structure, with 14 categories in the first level: business and industry, education, family and relationships, fitness and wellness, food and drink, hobbies and activities, lifestyle and culture, news and entertainment, people, shopping and fashion, sports and outdoors, technology, travel places and events, and null.

The interest extraction process done by Facebook relies on the information each user directly reveals as well as on proprietary inference on and off the platform. Users' interests are highly influenced by page likes and interests from other users with similar interests. In previous work, we have found six reasons for the assignment 
of interests and ad preferences ${ }^{79}$ : (i) "This is a preference you added", (ii) "You have this preference because we think it may be relevant to you based on what you do on Facebook, such as pages you've liked or ads you've clicked", (iii) "You have this preference because you clicked on an ad related to...", (iv) "You have this preference because you installed the app...", (v) "You have this preference because you liked a page related to...", (vi) "You have this preference because of comments, posts, shares or reactions you made related to...".

In order to define and query groups, location is the only field mandatory in the API. Other parameters are accepted and may be combined to obtain a more specific group. Facebook offers in all their APIs (including the Marketing API) unique identifiers for most parameter values, so values are language-independent. To help advertisers, the Marketing API also provides a search function to retrieve interests by string matching: given some string, it returns a list of full and partial matching name items, typically containing the unique interest ID, name, total number of users worldwide (reach within Facebook) and, sometimes, a brief description.

\section{Defining and downloading interests by populations}

To collect the data for our analysis we queried the Facebook Marketing API more than $75 \mathrm{M}$ times across the following geographical areas: 225 countries and territories, 413 subnational regions and 58 California counties. In addition, for some countries and regions we also obtained information by gender and age groups. When querying the Facebook Marketing API, we obtain the number of Monthly Active Users (MAU) and the number of Daily Active Users (DAU) for each interest and geographical location. To avoid daily fluctuations, we prefer to employ MAU over DAU. However, for privacy reasons, Facebook imposes a lower bound of 1,000 users for its MAU measure, so any unit with less than 1,000 users will be reported as 1,000. To mitigate this problem, whenever we have a unit of interest that is at its lower bound, we substitute MAU by DAU.

To obtain a comprehensive list of interests, we use the Marketing API's targeting search function that returns partially or fully matched interests by name when given a string query. To feed this function with meaningful and representative interests, we use all article titles in the English Wikipedia and all entries in the English dictionary. The Wikipedia titles are obtained from the DBpedia project (see https://wiki.dbpedia.org, last accessed August 1,2017), and the dictionary is the one contained in Ubuntu OS (Linux). This process yields 200 million records, including duplicates and non-interest parameters, such as demographics. After cleaning, the final number of unique interests is 399,182 . From that collection, we select all those with a potential reach of at least 500,000 users worldwide. While we employ this process for purposes of cross-national comparability, there is no theoretical reason why other scholars couldn't employ the list of all 400,000 interests, if they were interested in examining interests that were unique to particular populations. Our method leaves us with nearly 60,000 unique interest identifiers.

\section{Representativeness}

While most countries impose almost no restrictions on what content is available nor on how each citizen relates online, a few countries do limit the use of Facebook or the Internet. In the cases of Iran, Sudan and Cuba, the Marketing API does not provide information on people residing in these countries, returning a 2641 error with the following message: "By law, we cannot serve ads to the following countries." Another special case is China. Although it is possible to obtain user counts for China, Internet access to sites outside the country is severely restricted, making the figures reported by Facebook unrealistic. Hence, we do not include these countries in our analysis. 
We also collect age and gender data from the World Bank's World Development Indicators (WDI) and compare them to the age and gender structure of Facebook users by country. In the Supplementary Information we show that our qualitative findings are robust to limiting the analysis to either more representative or less representative subsamples.

\section{Facebook cultural distances}

Given data on the number of Facebook users and the number of users interested in each of the 59,763 Facebook interests in each of the 225 countries and territories, we construct a matrix of size (225 x 59,763) with the share of Facebook users that have a given interest in each country. Thus, the element in row $k$ and column $i$ of the matrix gives the share of Facebook users in location $k$ that have interest $i$, and each row vector has the shares of users for all interests for a given location.

The Facebook distance between two populations is computed as the cosine distance between the vectors of Facebook interest shares of the two populations. Consider two population groups, $k$ and $l$. The interests of population group $k$ can be represented by an $n$-dimensional vector $S_{k}$ with components $s_{i k}$ that measure the share of population $k$ that holds a particular interest $i$, where $i=1, \ldots, n$. Similarly, vector $S_{l}$ represents the interests of population group $l$. Denote the angle between $S_{k}$ and $S_{l}$ by $\theta$. The cosine distance between the interests of groups $k$ and $l$ is then:

$$
\cos \operatorname{dist}(k, l)=1-\cos (\theta)=1-\frac{S_{k} \cdot S_{l}}{\left\|S_{k}\right\|\left\|S_{l}\right\|}
$$

Since the cosine distance is based on the angle between two vectors, it does not depend on differences in the lengths of the vectors. In our context this is an advantage, because the norms of the vectors differ substantially across countries in a non-systematic way. For example, Spain and Italy, two countries that are similar in size, economic development and Facebook penetration, have vectors of very different lengths. There are many reasons why such differences may arise. For example, Facebook may be more easily able to identify interests in one country than in another, or people's intensity of using Facebook may differ across countries. Since we would not want such differences to be driving our measure of cultural distances, we want a distance measure that does not depend on the norms of the interest vectors. The cosine distance achieves this goal.

As an alternative, we could use the Euclidean distance, based on interest shares that are normalized by the

length of the interest vectors. After defining the normalized vector as $S_{k}{ }^{c}=\frac{S_{k}}{\left\|S_{k}\right\|}$, the normalized Euclidean distance is a simple transformation of the cosine distance:

$$
\text { norm euc dist }(k, l)=\left\|S_{k}^{\prime}-S_{l}^{\prime}\right\|=\sqrt{\left\|S_{k}^{\prime}\right\|^{2}+\left\|S_{l}^{\prime}\right\|^{2}-2\left\|S_{k}^{\prime}\right\|\left\|S_{l}^{\prime}\right\| \cos (\theta)}=\sqrt{2 \cos \operatorname{dist}(k, l)}
$$

The correlation between cosine distance and normalized Euclidean distance for the countries in our sample is 0.97 .

For the reasons mentioned above, other distance measures that do depend on differences between the lengths of the vectors may be less appropriate. However, reassuringly, even if we use Facebook distances based on other metrics (non-normalized Euclidean, Manhattan), these are highly correlated with cosine distances in our dataset $(\mathrm{r}>0.73, \mathrm{p}<0.0001)$. Figure S10 reports our main results based on Euclidean distances. Qualitatively, the results are unchanged. 
As is usual with correlations between distance matrices, all confidence intervals in this paper are based on Mantel tests ${ }^{80}$. All computations were done in Python 3.5 using distance measures implemented in scikits-learn version $0.19 .2^{81}$. In addition to calculating distances between countries, we also build matrices of Facebook distances for sub-national regions, age and gender groups within countries, as well as California counties.

\section{Other distance measures}

Cultural distances based on the World Values Survey are derived from 98 questions across 74 countries, spanning the period 1981-2010. Specifically, we follow Spoloare and Wacziarg ${ }^{12}$ and use the set of questions that is most common across countries and years, but unlike these authors we use cosine distance as the benchmark distance measure (the correlations among the various distances measures based on this set of questions for different metrics - cosine, Euclidean, Manhattan - is also very high: $r>0.97, p<0.0001$ ). In addition to distances based on all 98 questions, we also include a number of alternative measures of cultural distances, based on subsets of questions. These include measures for different macro-categories of questions (such as perceptions of life, family, work, etc.), as well as for binary and non-binary questions.

Genetic distances come from Spolaore and Wacziarg ${ }^{82}$ and are based on genetic data from Creanza et al. ${ }^{83}$. They measure population-weighted $F_{S T}$ genetic distances between countries. As additional measures, we also include $F_{S T}$ genetic distances between the plurality groups and genetic distances as they were in the year 1500 , as well as additional distance measures based on Spoloare and Wacziarg ${ }^{12}$.

Geographic distances measure distances between capitals. We also include a number of alternative measures: distances between the most populated cities, population-weighted distances, and population-weighted distances that account for the sensitivity of trade flows to distance. All these measures come from the CEPII GeoDist database ${ }^{84}$.

Linguistic distances measure the distance between two randomly drawn individuals of two different countries, where the linguistic distance is based on the Ethnologue language tree and uses the formula by Desmet, Ortuño-Ortín and Wacziarg ${ }^{26}$. We also include alternative measures of linguistic distances taken from Spolaore and Wacziarg ${ }^{82}$ : 15 additional measures where languages are defined at 15 different levels of aggregation (e.g., at aggregation level 1 all Indo-European languages are taken to be the same), and two distance measures based on cognates, one for plurality languages and another weighted by the language shares.

The measure for religious distances is based on population-weighted distances of religions using a religion tree from the World Christian Database ${ }^{12}$. We also include alternative measures: a distance measure based on plurality groups, and two measures based on an alternative religion tree from Mecham, Fearon and Laitin ${ }^{85}$.

\section{Principal component analysis}

We perform principal component analysis on Facebook interests and on WVS questions, using the common sample of countries covered by both data sources. The goal is to reduce the dimensionality of interests and questions, and assess whether a limited number of principal components are able to explain a large share of the variance in Facebook interests across countries and a large share of the variance of WVS questions across countries. When conducting principal component analysis, we use the covariance matrix, since our variables are population shares, measured on the same scale, from 0 to 1 . In the Supplementary Information we show that the results are similar when using the correlation matrix instead. 


\section{Dendrograms}

To generate the dendrograms, we use an implementation of hierarchical agglomerative clustering (HAC) that is provided by the python package scipy.cluster.hierarchy. This implementation of HAC allows for a choice of a distance method and a linkage criterion. We use cosine distance for consistency across other analyses and employ the Ward variance minimization linkage criterion. The input is the interest share vector of each country.

Hierarchical clustering algorithms are a general family of clustering algorithms that build nested clusters by merging or splitting the clusters successively in iterations. The nested structure can be represented as a dendrogram, in which the root of the tree is the highest level cluster that includes all data, while the leaf of the tree represents the lowest level cluster that includes a single data point.

Hierarchical clustering can be performed through a bottom-up (agglomerative) strategy or a top-down (divisive) strategy. In the agglomerative strategy, hierarchical clustering is performed via a bottom-up process in which every data point starts as its own cluster. The algorithm builds nested clusters by merging or splitting the clusters successively in multiple iterations and represents the nested structure as a dendrogram.

Adopting notations from Arbelaitz et al. ${ }^{86}$, a data set DS is defined as a set of $\mathrm{N}$ data points, each of which is a vector of $\mathrm{F}$ dimensions. A clustering of DS is a set of disjoint clusters that partitions DS into K groups

$$
C=\left\{c_{1}, c_{2}, \ldots, c_{K}\right\} \text { where } \forall K>1 \cup_{c_{k} \in C} c_{k}=D S \text { and } \cap_{c_{k} \in C} c_{k}=\varnothing
$$

In the first iteration, $\left(\begin{array}{l}K \\ 2\end{array}\right)$ distance values between base $\mathrm{K}$ clusters are computed using a distance metric. After the distance values have been evaluated, a linkage criteria is used to determine two clusters $c_{i}$ and $c_{j}$ to combine to form a new cluster $c_{k}$. The Ward variance minimization linkage criterion is given by the following formula:

$$
d\left(c_{k}, c_{l}\right)=\sqrt{\frac{\left|c_{l}\right|+\left|c_{i}\right|}{T} d\left(c_{l}, c_{i}\right)^{2}+\frac{\left|c_{l}\right|+\left|c_{j}\right|}{T} d\left(c_{l}, c_{j}\right)^{2}-\frac{\left|c_{l}\right|}{T} d\left(c_{i}, c_{j}\right)^{2}}
$$

where $c_{k}$ is a newly formed cluster consisting of clusters $c_{i}$ and $c_{j}, c_{l}$ is an unused cluster, $T=\left|c_{l}\right|+\left|c_{i}\right|+\left|c_{j}\right|$ and $|\cdot|$ is the cardinality of its argument.

\section{Dendrogram of full set of countries}

Figure 3 depicts a dendrogram based on the set of 72 countries common to both the WVS and Facebook sample. Supplemental Material Figure S13 shows a dendrogram for the full set of 225 countries for which we have Facebook interest shares.

Regional, gender, and age divisiveness

Our measure of regional divisiveness is as follows. Consider a country $c$ with a number of regions indexed by $k$ or $l$. Denote the Facebook cosine distance between any two regions $k$ and $l$ by cos dist $(k, l)$, and denote the population shares of $k$ and $l$ by $s_{k}$ and $s_{l}$. We then measure the regional divisiveness of country $c$ as the population-weighted Facebook cosine distance between its different subnational regions:

$$
\frac{\sum_{k \neq l} \sum_{l} \cos \operatorname{dist}(k, l) s_{k} s_{l}}{\sum_{k \neq l} \sum_{l} s_{k} s_{l}}
$$

One way of interpreting (3) is as the expected Facebook distance between two randomly drawn individuals from 
different regions. If there are only two regions in a country, then (3) would simplify to the bilateral distance between them, i.e., $\cos \operatorname{dist}(k, l)$. More simply, (3) is an average interregional distance measure. Gender and age divisiveness are measured similarly.

Country networks and maps

From the 18 countries for we downloaded regional data, we chose Germany and India to show network and map, given their rank according to divisiveness in Figure 6A. For each country, we first constructed a network in which each node represents a region, and we used links to connect every pair of regions. Links are weighted by the cosine similarity between regions ( 1 - cosine distance). Link weights are rescaled using standardization and then shifted by a constant value as to have only non-negative weights (a condition on the input for the community detection method). Then, to detect communities, we used an igraph (from $R$ ) implementation of multi-level modularity optimization algorithm (also known as Louvain method) ${ }^{72}$, which is based on the modularity measure and a hierarchical approach. Modularity is a measure of a quality of a partition (codomain: [-1, 1]), which measures the density of links inside communities as compared to links between communities ${ }^{87,88}$. Then, we colored nodes according to community affiliation, and links according to their adjacent nodes. To plot country maps, we used $R$ package rnaturalearth to access geometry of regions' layout from Natural Earth map data $^{89}$. We colored map regions according to the communities detected from the corresponding network.

\section{References}

1. Boyd, R. \& Richerson, P. J. The Origin and Evolution of Cultures. (Oxford University Press, 2005).

2. Kempe, M., Lycett, S. J. \& Mesoudi, A. From cultural traditions to cumulative culture: parameterizing the differences between human and nonhuman culture. J. Theor. Biol. 359, 29-36 (2014).

3. Mesoudi, A. \& Thornton, A. What is cumulative cultural evolution? Proc. Biol. Sci. 285, (2018).

4. Dean, L. G., Kendal, R. L., Schapiro, S. J., Thierry, B. \& Laland, K. N. Identification of the social and cognitive processes underlying human cumulative culture. Science 335, 1114-1118 (2012).

5. Baldwin, J. R., Faulkner, S. L., Hecht, M. L. \& Lindsley, S. L. Redefining Culture: Perspectives Across the Disciplines. (Routledge, 2006).

6. Harris, M. \& University of Florida. Cultural Materialism: The Struggle for a Science of Culture. (AltaMira Press, 2001).

7. Goldstein, L. J. On Defining Culture. Am. Anthropol. 59, 1075-1081 (1957).

8. Swidler, A. Culture in Action: Symbols and Strategies. Am. Sociol. Rev. 51, 273-286 (1986).

9. Chilton, S. Defining Political Culture. West. Polit. Q. 41, 419-445 (1988).

10. Ross, M. H. Culture in Comparative Political Analysis. In: Comparative Politics: Rationality, Culture, and Structure 134-161 (Cambridge University Press, 2009). doi:10.1017/CBO9780511804007.007. 
11. Huntington, S. P. The Clash of Civilizations and the Remaking of World Order. (Penguin Books India, 1997).

12. Spolaore, E. \& Wacziarg, R. Ancestry, language and culture. in The Palgrave handbook of economics and language 174-211 (Springer, 2016).

13. Oosterbeek, H., Sloof, R. \& van de Kuilen, G. Cultural Differences in Ultimatum Game Experiments: Evidence from a Meta-Analysis. (2001) doi:10.2139/ssrn.286428.

14. Ottaviano, G. I. P. \& Peri, G. The Economic Value of Cultural Diversity: Evidence from US Cities. NBER WP 10904, (2004) doi:10.3386/w10904.

15. Tomasello, M. The Cultural Origins of Human Cognition.(Harvard University Press, 2009).

16. Cohen, A. B. Many forms of culture. Am. Psychol. 64, 194-204 (2009).

17. Betancourt, H. \& López, S. R. The study of culture, ethnicity, and race in American psychology. Am. Psychol. 48, 629-637 (1993).

18. Mesoudi, A. Cultural evolution: integrating psychology, evolution and culture. Current Opinion in Psychology 7, 17-22 (2016).

19. Ekman, P., Friesen, W. V., O’Sullivan, M. \& Chan, A. Universals and cultural differences in the judgments of facial expressions of emotion. J. Pers. Soc. Psychol. 53, 712-717 (1987).

20. Moore, O. K. Nominal Definitions of 'Culture'. Philos. Sci. 19, 245-256 (1952).

21. Alesina, A. \& Spolaore, E. On the number and size of nations. Q. J. Econ. 112, 1027-1056 (1997).

22. Alesina, A., Spolaore, E. \& Wacziarg, R. Economic integration and political disintegration. Am. Econ. Rev. 90, 1276-1296 (2000).

23. Desmet, K., Le Breton, M., Ortuño-Ortín, I. \& Weber, S. The stability and breakup of nations: a quantitative analysis. J. Econ. Growth 16, 183 (2011).

24. Fearon, J. D. \& Laitin, D. D. Ethnicity, insurgency, and civil war. Am. Polit. Sci. Rev. 97, 75-90 (2003).

25. Fearon, J. D. Ethnic and cultural diversity by country. J. Econ. Growth 8, 195-222 (2003).

26. Desmet, K., Ortuño-Ortín, I. \& Wacziarg, R. Culture, ethnicity, and diversity. Am. Econ. Rev. 107, 2479-2513 (2017).

27. Alesina, A. \& Ferrara, E. L. Ethnic diversity and economic performance. J. Econ. Lit. 43, 762-800 (2005).

28. Thomson, R. et al. Relational mobility predicts social behaviors in 39 countries and is tied to historical farming and threat. Proc. Natl. Acad. Sci. U. S. A. 115, 7521-7526 (2018).

29. Taras, V., Rowney, J. \& Steel, P. Half a century of measuring culture: Review of approaches, challenges, and limitations based on the analysis of 121 instruments for quantifying culture. Journal of International Management 15, 357-373 (2009). 
30. Lazer, D. et al. Life in the network: the coming age of computational social science. Science 323, 721 (2009).

31. van der Post, D. J. \& Hogeweg, P. Diet traditions and cumulative cultural processes as side-effects of grouping. Anim. Behav. 75, 133-144 (2008).

32. Tamariz, M. \& Kirby, S. The cultural evolution of language. Curr Opin Psychol 8, 37-43 (2016).

33. Kirby, S., Dowman, M. \& Griffiths, T. L. Innateness and culture in the evolution of language. Proc. Natl. Acad. Sci. U. S. A. 104, 5241-5245 (2007).

34. Arendt, H. Reflections on literature and culture. (Stanford University Press, 2007).

35. Acerbi, A., Enquist, M. \& Ghirlanda, S. Cultural evolution and individual development of openness and conservatism. Proc. Natl. Acad. Sci. U. S. A. 106, 18931-18935 (2009).

36. Atran, S. \& Henrich, J. The Evolution of Religion: How Cognitive By-Products, Adaptive Learning Heuristics, Ritual Displays, and Group Competition Generate Deep Commitments to Prosocial Religions. Biol. Theory 5, 18-30 (2010).

37. Geertz, C. The Interpretation Of Cultures. (Basic Books, 1973).

38. Murthy, D. Digital Ethnography: An Examination of the Use of New Technologies for Social Research. Sociology 42, 837-855 (2008).

39. Henrich, J. Demography and Cultural Evolution: How Adaptive Cultural Processes Can Produce Maladaptive Losses-The Tasmanian Case. Am. Antiq. 69, 197-214 (2004).

40. Posner, D. N. The Political Salience of Cultural Difference: Why Chewas and Tumbukas are Allies in Zambia and Adversaries in Malawi. Am. Polit. Sci. Rev. 98, 529-545 (2004).

41. Murray, J. H. Toward a Cultural Theory of Gaming: Digital Games and the Co-Evolution of Media, Mind, and Culture. Popular Communication 4, 185-202 (2006).

42. Center, P. R. Social media fact sheet. Pew Research Center: Internet, Science $\mathcal{E}$ Tech (2017).

43. Bontempo, R. N., Bottom, W. P. \& Weber, E. U. Cross-Cultural Differences in Risk Perception: A ModelBased Approach. Risk Anal. 17, 479-488 (1997).

44. Yuki, M., Maddux, W. W., Brewer, M. B. \& Takemura, K. Cross-cultural differences in relationship- and group-based trust. Pers. Soc. Psychol. Bull. 31, 48-62 (2005).

45. Schimmack, U., Oishi, S. \& Diener, E. Individualism: a valid and important dimension of cultural differences between nations. Pers. Soc. Psychol. Rev. 9, 17-31 (2005).

46. Archer, J. Cross-cultural differences in physical aggression between partners: a social-role analysis. Pers. Soc. Psychol. Rev. 10, 133-153 (2006). 
47. Segall, M. H., Campbell, D. T. \& Herskovits, M. J. Cultural Differences in the Perception of Geometric Illusions. Science 139, 769-771 (1963).

48. Shenkar, O. Cultural Distance Revisited: Towards a More Rigorous Conceptualization and Measurement of Cultural Differences. Journal of International Business Studies 32, 519-535 (2001).

49. Hofstede, G. National Cultures in Four Dimensions: A Research-Based Theory of Cultural Differences among Nations. International Studies of Management $\mathcal{F}$ Organization 13, 46-74 (1983).

50. Inglehart, R. et al. World Values Survey: All Rounds - Country-Pooled Datafile Version: http://www.worldvaluessurvey.org/ (JD Systems Institute, 2014).

51. Geertz, C. Thick description: Toward an interpretive theory of culture. in The Cultural Geography Reader 41-51 (Routledge, 2008).

52. Fisher, R. J. Social Desirability Bias and the Validity of Indirect Questioning. J. Consum. Res. 20, 303-315 (1993).

53. McCambridge, J., Witton, J. \& Elbourne, D. R. Systematic review of the Hawthorne effect: new concepts are needed to study research participation effects. J. Clin. Epidemiol. 67, 267-277 (2014).

54. Brewer \& John. Ethnography. (McGraw-Hill Education (UK), 2000).

55. Zhang, Y. \& Neelankavil, J. P. The influence of culture on advertising effectiveness in China and the USA. Eur. J. Mark. 31, 134-149 (1997).

56. Hong, J. W., Muderrisoglu, A. \& Zinkhan, G. M. Cultural Differences and Advertising Expression: A Comparative Content Analysis of Japanese and U.S. Magazine Advertising. J. Advert. 16, 55-68 (1987).

57. Chau, P. Y. K., Cole, M., Massey, A. P., Montoya-Weiss, M. \& O’Keefe, R. M. Cultural differences in the online behavior of consumers. Commun. ACM 45, 138-143 (2002).

58. Kosinski, M., Stillwell, D. \& Graepel, T. Private traits and attributes are predictable from digital records of human behavior. Proc. Natl. Acad. Sci. U. S. A. 110, 5802-5805 (2013).

59. Kosinski, M., Matz, S. C., Gosling, S. D., Popov, V. \& Stillwell, D. Facebook as a research tool for the social sciences: Opportunities, challenges, ethical considerations, and practical guidelines. Am. Psychol. 70, 543-556 (2015).

60. Muthukrishna, M. et al. Beyond WEIRD Psychology: Measuring and Mapping Scales of Cultural and Psychological Distance. Psychological Science (2020) doi:10.2139/ssrn.3259613.

61. Cavalli-Sforza, L. L. \& Feldman, M. W. Cultural transmission and evolution: a quantitative approach. (Princeton University Press, 1981).

62. Cavalli-Sforza, L. L., Menozzi, P. \& Piazza, A. The history and geography of human genes. (Princeton University Press, 1994). 
63. Cavalli-Sforza, L. L. Genes, peoples, and languages. (North Point Press, 2000).

64. Atkinson, Q. D. Phonemic diversity supports a serial founder effect model of language expansion from Africa. Science 332, 346-349 (2011).

65. Rogers, D. S., Feldman, M. W. \& Ehrlich, P. R. Inferring population histories using cultural data. Proceedings of the Royal Society of London B: Biological Sciences 276, 3835-3843 (2009).

66. Ramachandran, S. et al. Support from the relationship of genetic and geographic distance in human populations for a serial founder effect originating in Africa. Proc. Natl. Acad. Sci. U. S. A. 102, 15942-15947 (2005).

67. Diamond, J. M. Guns, germs, and steel: the fates of human societies. (W.W. Norton \& Co., 1997).

68. Henrich, J. P. The secret of our success: how culture is driving human evolution, domesticating our species, and making us smarter. (Princeton University Press, 2016).

69. Richerson, P. J. \& Christiansen, M. H. Cultural evolution: society, technology, language, and religion. (MIT Press, 2013).

70. Galor, O. \& Özak, Ö. The Agricultural Origins of Time Preference. Am. Econ. Rev. 106, (2016).

71. Inglehart, R. et al. World Values Survey: Round Six - Country-Pooled Datafile Version: http://www.worldvaluessurvey.org/l (JD Systems Institute, 2014).

72. Blondel, V. D., Guillaume, J.-L., Lambiotte, R. \& Lefebvre, E. Fast unfolding of communities in large networks. Journal of Statistical Mechanics: Theory and Experiment vol. 2008 P10008 (2008).

73. Bail, C. A. The cultural environment: measuring culture with big data. Theory Soc. 43, 465-482 (2014).

74. Michel, J.-B. et al. Quantitative analysis of culture using millions of digitized books. Science 331, 176-182 (2011).

75. Reeves, B., Robinson, T. \& Ram, N. Time for the Human Screenome Project. Nature 577, 314-317 (2020).

76. Mesoudi, A. Pursuing Darwin's curious parallel: Prospects for a science of cultural evolution. Proc. Natl. Acad. Sci. U. S. A. 114, 7853-7860 (2017).

77. Hebert, P. D. N., Cywinska, A., Ball, S. L. \& deWaard, J. R. Biological identifications through DNA barcodes. Proc. Biol. Sci. 270, 313-321 (2003).

78. Englehardt, S. \& Narayanan, A. Online Tracking. Proceedings of the 2016 ACM SIGSAC Conference on Computer and Communications Security - CCS'16 (2016) doi:10.1145/2976749.2978313.

79. Cabañas, J. G., Cuevas, Á. \& Cuevas, R. Unveiling and Quantifying Facebook Exploitation of Sensitive Personal Data for Advertising Purposes. (2018).

80. Legendre, P. \& Legendre, L. Numerical ecology. vol. 24 (Elsevier, 2012). 
81. Pedregosa, F. et al. Scikit-learn: Machine learning in Python. J. Mach. Learn. Res. 12, 2825-2830 (2011).

82. Spolaore, E. \& Wacziarg, R. Ancestry and development: New evidence. J. Appl. Econometrics 33, 748-762 (2018).

83. Creanza, N. et al. A comparison of worldwide phonemic and genetic variation in human populations. Proceedings of the National Academy of Sciences 112, 1265-1272 (2015).

84. Mayer, T. \& Zignago, S. Notes on CEPII's distances measures: The GeoDist database. (2011).

85. Mecham, R. Q., Fearon, J. \& Laitin, D. Religious classification and data on shares of major world religions. unpublished, Stanford University 1, 18 (2006).

86. Arbelaitz, O., Gurrutxaga, I., Muguerza, J., Pérez, J. M. \& Perona, I. An extensive comparative study of cluster validity indices. Pattern Recognit. 46, 243-256 (2013).

87. Girvan, M. \& Newman, M. E. J. Community structure in social and biological networks. Proceedings of the National Academy of Sciences vol. 99 7821-7826 (2002).

88. Newman, M. E. J. Modularity and community structure in networks. Proceedings of the National Academy of Sciences vol. 103 8577-8582 (2006).

89. Natural Earth. http://www. naturalearthdata.com.

90. World Bank. World Development Indicators.

91. United Nations. Department of Economic and Social Affairs, Population Division. World Marriage Data 2015, POP/DB/Marr/Rev2015, (2015).

92. World Health Organization. Global Health Observatory data repository.

93. United Nations Food and Agricultural Organization (FAO). FAOSTAT.

94. Georgetown Institute for Women, Peace and Security Contact (GIWPS). WPSIndex.

95. United Nations Development Programme (UNDP). Human Development Report 2019.

96. World Economic Forum. Global Gender Gap Report 2020.

97. Helliwell, J., Layard, R., \& Sachs, J. World Happiness Report 2019. (New York: Sustainable Development Solutions Network, 2019).

98. World Health Organization. Global Health Observatory (GHO) data.

99. World Economic Forum. Global Competitiveness Index.

100. Enke, B. Kinship, cooperation, and the evolution of moral systems. Quarterly Journal of Economics 134, 953-1019 (2019). 
101. Falk, A., Becker, A., Dohmen, T., Enke, B., Huffman, D., \& Sunde, U. Global evidence on economic preferences. Quarterly Journal of Economics 133, 1645-1692 (2018).

102. Falk, A., Becker, A., Dohmen, T., Huffman, D., \& Sunde, U. The preference survey module: A validated instrument for measuring risk, time, and social preferences. IZA Discussion Paper No. 9674 (2016).

103. Hofstede, G., Hofstede, G. J. \& Minkov, M. Cultures and organizations: software of the mind. (New York: McGraw-Hill USA, 2010). 


\section{Supplementary Information}

\section{Partial correlations of distance measures}

To further explore whether Facebook distances capture cultural distances, we look at various partial correlations. That is, we analyze the correlation between one distance measure (e.g., WVS distances) and the Facebook distance measure, controlling for all other distance measures (e.g., genetic, geographic, linguistic and religious distances). The goal is to discover which type of distance measure correlates most strongly with FB distances.

We start by focusing on one measure for each type of distance proxy. That is, rather than using different ways of measuring each distance proxy, we choose one measure for each one of the five distance proxies (values, genetic, geographic, linguistic, and religious). ${ }^{1}$ Before looking at partial correlations, Figure S1(a) plots the correlations between Facebook distances and each one of the distance measures, not controlling for any other distance. The correlations of Facebook distances with genetic, geographic, linguistic and religious distances are all positive and statistically significant at the $95 \%$ confidence level, but the strongest correlation continues to be with the most direct survey-based measure of cultural distances. ${ }^{2}$ This confirms that our bottom-up Facebook measure of cultural distance corresponds well to the standard top-down measure of cultural distance.

Figure S1(b) plots the partial correlations between Facebook distances and each one of the distance measures. To give a specific example, consider the partial correlation between Facebook distances and value-based cultural distances (slightly above 0.35 ). This number represents the correlation between the residuals of a regression of Facebook distances on all other distances (genetic, geographic, linguistic and religious) and the residuals of a regression of value-based cultural distances on all other distances (genetic, geographic, linguistic and religious). It hence tells us how correlated Facebook and value-based cultural distances are, after controlling for all other distances. The same partial correlations with other distances are all lower: for example, the partial correlation between Facebook and geographic distances is below 0.1, and not statistically significant at the $95 \%$ confidence level. Hence, when controlling for all other distances, the strongest partial correlation is between Facebook distances and value-based cultural distances. This shows that Facebook distances are not just picking up geographic, genetic, linguistic or religious distances.

The other two panels of Figure S1 confirm this finding. Panel (c) shows the semi-partial correlations between Facebook distances and each one of the distance measures. This represents the correlations between Facebook distances and the residuals of a regression of one of the distance measures on all other distance measures. Once again, we find that the strongest semi-partial correlation is with survey-based cultural distances. Panel (d) reports the semi-partial $\mathrm{R}^{2}$ of Facebook distances on each one of the distance measures, after controlling for all others. For example, the semi-partial $\mathrm{R}^{2}$ of 0.085 between Facebook and value-based cultural distances means that a regression of Facebook distances on all other distance measures (including value-based cultural distances) explains $8.5 \%$ more of the variation in Facebook distances than a regression that excludes value-based cultural distances.

\footnotetext{
${ }^{1}$ In particular, value distances are based on 98 questions from the World Values Survey, spanning the period 1981- 2000, as in 12 , with the only difference that we use cosine distance; genetic distances come from ${ }^{82}$ and measure population-weighted $\mathrm{F}_{\mathrm{ST}}$ genetic distances between countries using genetic data by ${ }^{83}$; geographic distances are between country capitals; linguistic distances are based on the Ethnologue database and use the formula by ${ }^{26}$ to measure the linguistic distance between two randomly drawn individuals of two different countries; and religious distances are based on the population-weighted distance using a religion tree from the World Christian Database ${ }^{12}$.

${ }^{2}$ As is usual with correlations between distance matrices, all confidence intervals are based on Mantel tests ${ }^{80}$.
} 


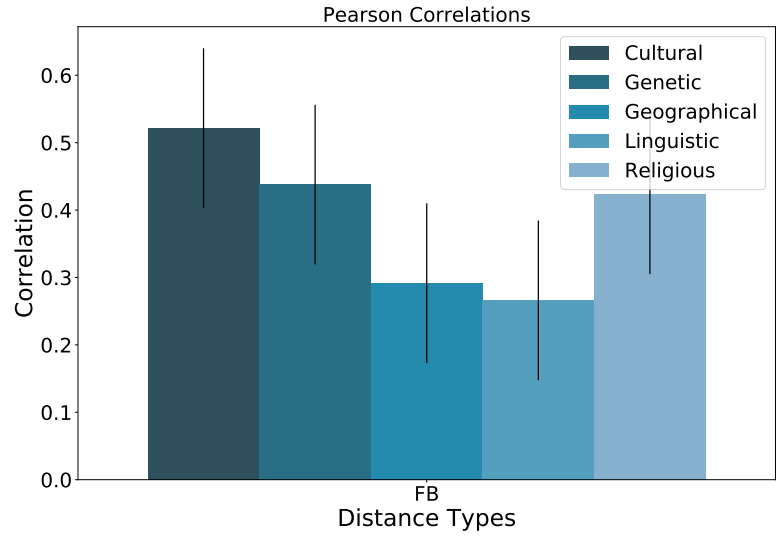

(a) Correlation

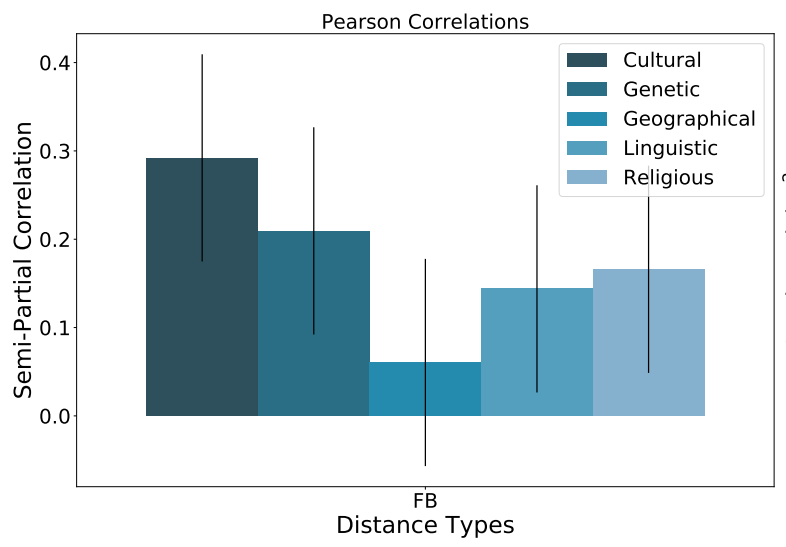

(c) Semi-Partial Correlation (Actual on Residuals)

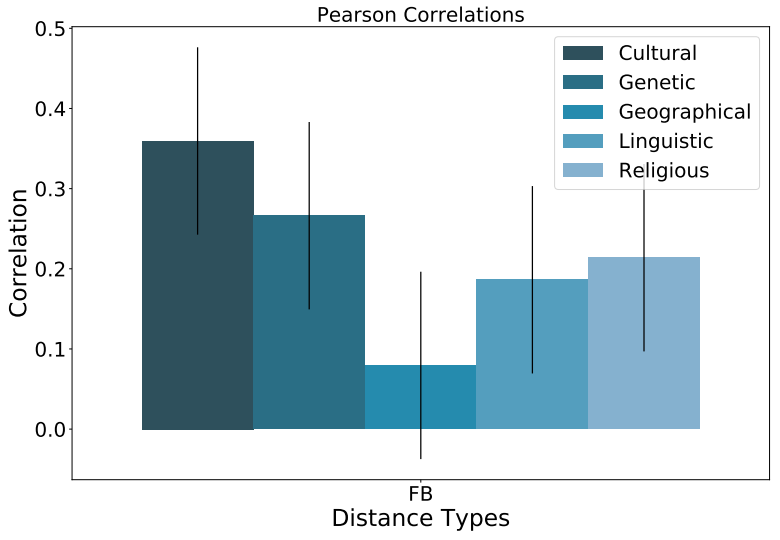

(b) Partial Correlation (Residuals on Residuals)

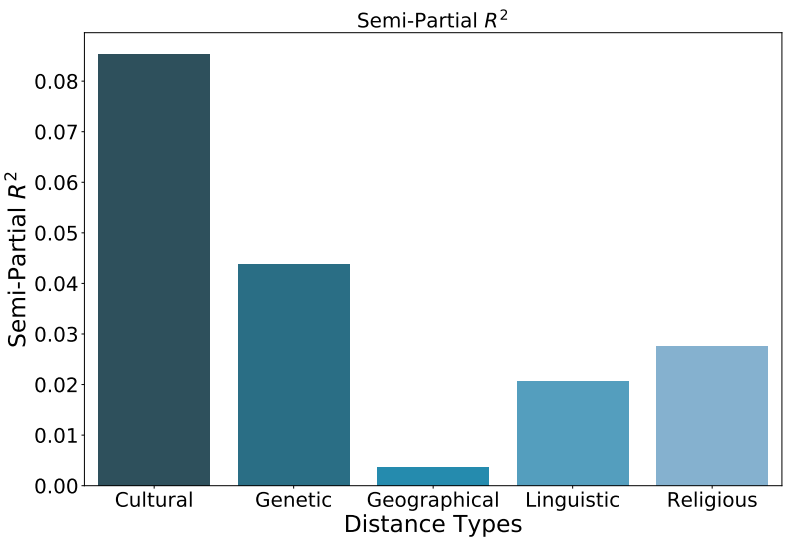

(d) Semi-Partial $R^{2}$

Figure S1: Correlations Between Facebook and Selected Distance Measures. Panel (a) plots the correlations between Facebook distances and each one of the distance measures, not controlling for any other distance. Panel (b) plots the partial correlations between Facebook distances and each one of the distance measures. For example, the partial correlation between Facebook distances and value-based distances corresponds to the correlation between the residuals of a regression of Facebook distances all other distances (genetic, geographic, linguistic and religious) and the residuals of a regression of value-based cultural distances on all other distances (genetic, geographic, linguistic and religious). Panel (c) plots the semi-partial correlations between Facebook distances and each one of the distance measures. For example, the semi-partial correlation between Facebook distances and value-based distances corresponds to the correlation between Facebook distances and the residuals of a regression of value-based cultural distances on all other distances (genetic, geographic, linguistic and religious). Panel (d) plots the semi-partial $R^{2}$ of Facebook distances on each one of the distance measures. For example, the semi-partial $R^{2}$ between Facebook and value-based cultural distances corresponds to the difference in $R^{2}$ of a regression of Facebook distances on all other distance measures (including value-based cultural distances) and a regression of Facebook distances on all other distance measures (excluding value-based cultural distances). The distance measures for each proxy (value-based, genetic, geographic, linguistic and religious) are the ones given in footnote 1. 


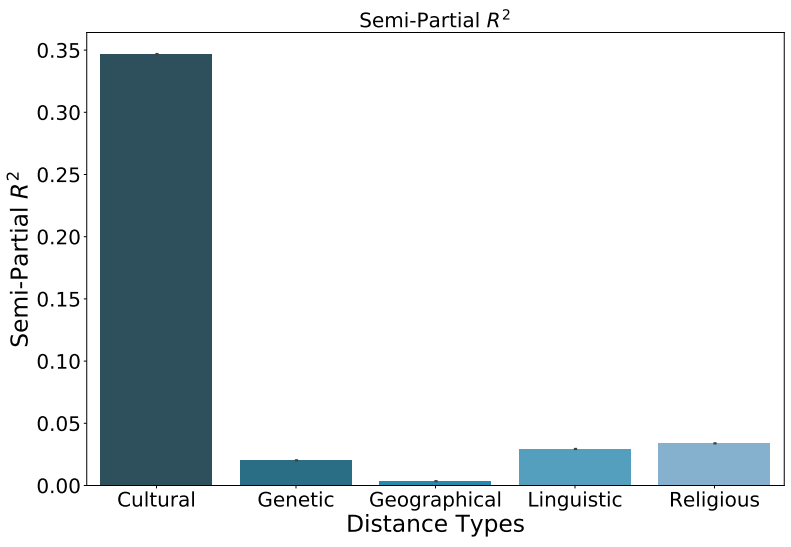

(a) Semi-Partial $R^{2}$ (Joint)

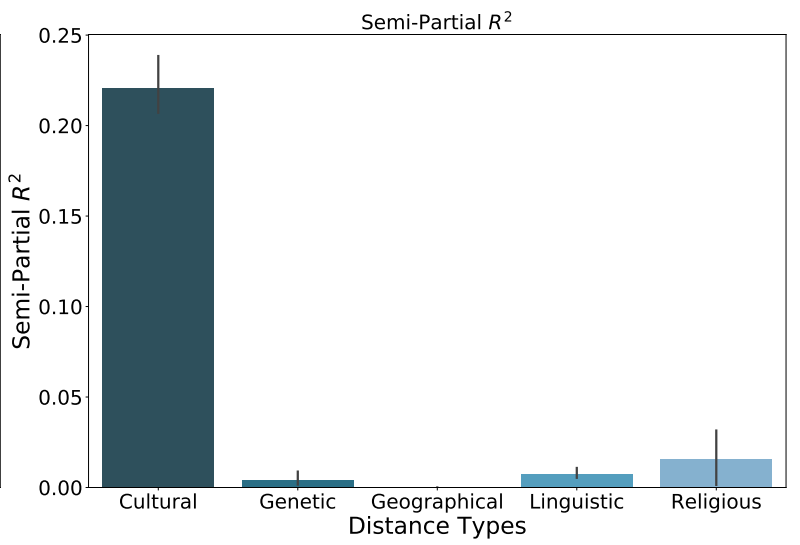

(b) Semi-Partial $R^{2}$ (Individual)

Figure S2: Semi-Partial $\boldsymbol{R}^{2}$ of Facebook Distance on All Distance Measures. Panel (a) plots the semi-partial $R^{2}$ of Facebook distances on all alternative measures of each one of the distance proxies. For example, the semi-partial $R^{2}$ between Facebook and value-based cultural distances corresponds to the difference in $R^{2}$ of a regression of Facebook distances on all alternative distance measures of the five proxies (including all alternative measures of value-based cultural distances) and a regression of Facebook distances on all alternative measures of the distance proxies (excluding all alternative measures of value-based cultural distances). Panel (b) plots the average semi-partial $R^{2}$ of Facebook distances on alternative measures of each one of the distance proxies. For example, the average semi-partial $R^{2}$ between Facebook and value-based cultural distances corresponds to the average difference in $R^{2}$ of a regression of Facebook distances on all alternative distance measures of the proxies (including one of the measures of value-based cultural distances) and a regression of Facebook distances on all alternative measures of the distance proxies (excluding value-based cultural distances). The alternative distance measures for each proxy (value-based, genetic, geographic, linguistic and religious) are the ones given in Figure 2 of the main paper, and further described in the Methods section.

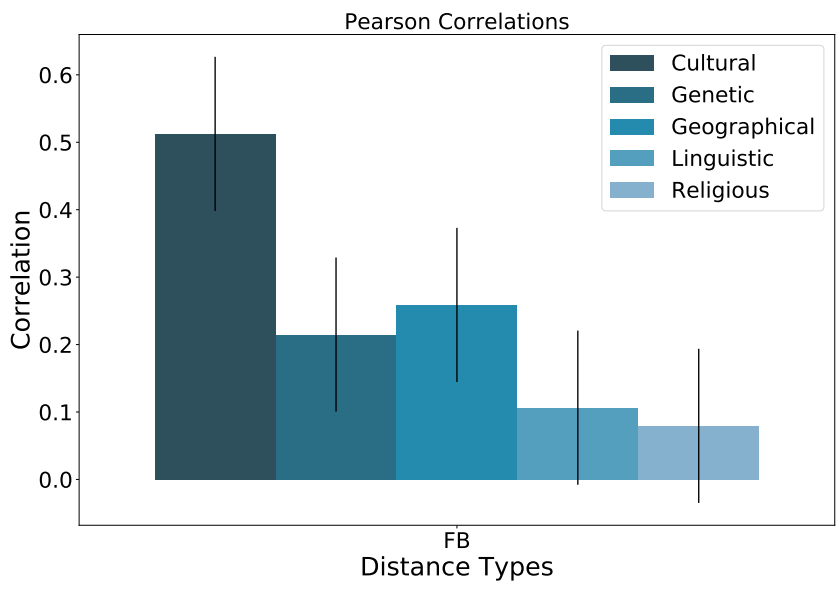

Figure S3: Correlations Between Facebook and Selected Distance Measures, Using Full Sample. This figure plots the correlation between Facebook distances and select measures of each of the distance proxies (value-based, genetic, geographic, linguistic and religious). It uses the full sample of countries, rather than the sample that is common to all measures. The select measures of the distance proxies are the ones given in footnote 1.

We now turn to using more than one measure for each type of distance proxy. As in the main paper, we use all alternative measures of the five distance proxies (values, genetic, geographic, linguistic, and religious). Figure S2 reports the results. To explain the difference between the graphs, we focus on the first bar of each graph. The first bar in Panel (a) shows the semi-partial $\mathrm{R}^{2}$ of a regression of Facebook distances on all valuebased cultural distances, after controlling for all other measures of distance (genetic, geographic, linguistic and 
religious). The first bar in Panel (b) shows the average semi-partial $\mathrm{R}^{2}$ of a regression of Facebook distances on each value-based cultural distance separately, after controlling for all other measures of distance (genetic, geographic, linguistic and religious). All other bars in the two panels show the same information, but for genetic, geographic, linguistic and religious distances. These graphs confirm our main finding: although all distance measures partly explain Facebook distances, survey-based cultural distances have the strongest explanatory power.

\section{Robustness to alternative samples}

In this section we explore the robustness of our analysis to various samples. First, Figure S3 plots the correlation between Facebook distances and select measures of each of the distance proxies, using the full sample of countries rather than the common sample. The results confirm that the strongest correlation with Facebook distances are value-based distances from the WVS. Second, Figure S4 shows the results of replicating the main analysis when we constrain the sample to countries with more than 300,000 people and a Facebook penetration above 5\%. This decreases the number of countries for which we have Facebook distances from 225 to 161. The results are quantitatively and qualitatively very similar. This is not surprising: most of the countries that drop out were not in the common sample.

Third, we explore to what extent the representativity of Facebook users affects our findings. For this analysis, we start by comparing, for each country, its Facebook user composition in terms of gender to the composition of its actual gender composition using data from the World Development Indicators in 2017. Starting from the common sample, we create two groups of countries: a first group of countries that are above the median difference in gender composition when comparing Facebook users and the actual population, and a second group of countries that are below the median difference in age composition. In the first group Facebook is less representative of the population than in the second group. Figures S5 and S6 show the results of splitting the sample based on gender composition. Reassuringly the results are similar to the main analysis.

Next, we explore the representativity in terms of age composition. Focusing on the population aged 15-64, we compare the share of FB users aged 15-29 to the share of the actual population aged 15-29. We select this age split because on average about half of the Facebook users aged 15-64 are in the group 15-29. As before, we create two groups of countries: a less representative group of countries that are above the median difference in age composition when comparing Facebook users and the actual population, and a more representative group of countries that are below the median difference in age composition. Figures S7 and S8 show that similar results are obtained if we split the sample based on age composition.

In addition to exploring robustness to different samples of countries, we also analyze robustness to different samples of interest categories. Figure S9 plots the correlation between Facebook distances and each one of the distance measures for each one of the 14 macro-categories of interests: people; lifestyle and culture; travel, places and events; empty; hobbies and activities; news and entertainment; shopping and fashion; business and industry; food and drink; sports and outdoors; education; technology; fitness and wellness; and family and relationships. Quite a few interests are marked by Facebook as a local business. To ensure differences between countries are not driven by such local businesses, we add one additional robustness check that focuses exclusively on interests that are not marked as local businesses. As can be seen in Figure S9, the results do not differ substantially across these different sub-samples of interests. 


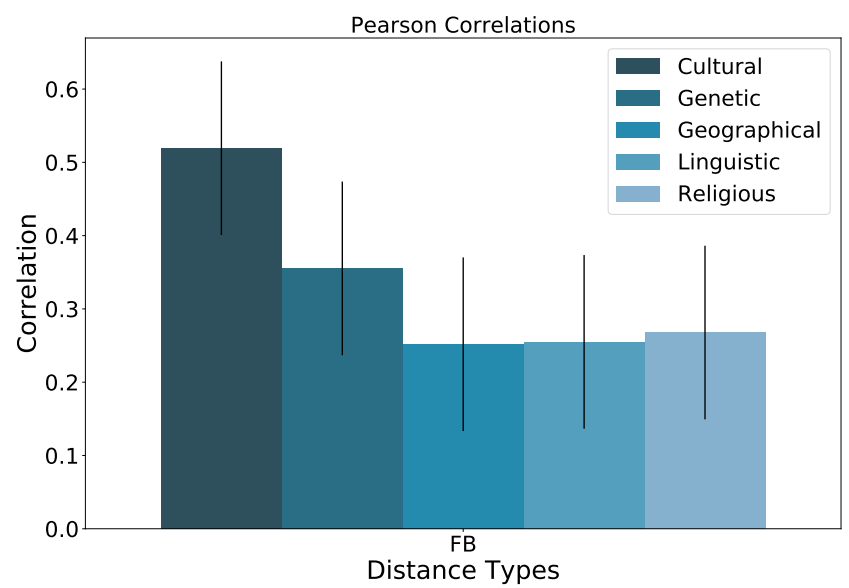

(a) Full Sample

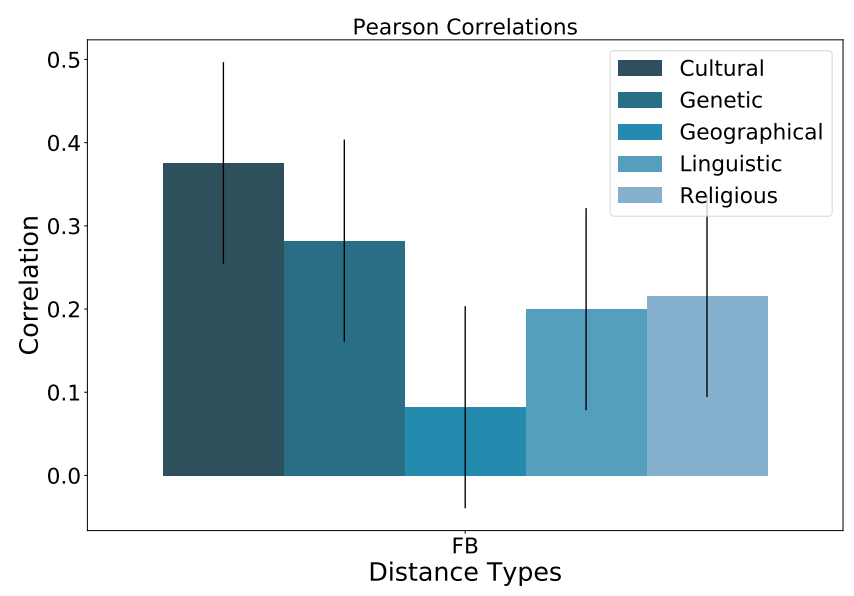

(c) Partial Correlation (Residuals on Residuals)

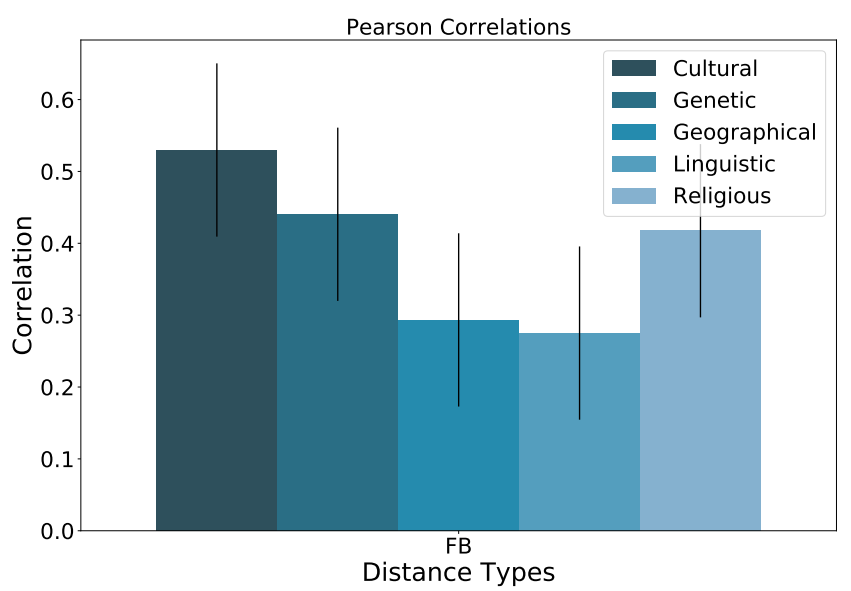

(b) Common Sample

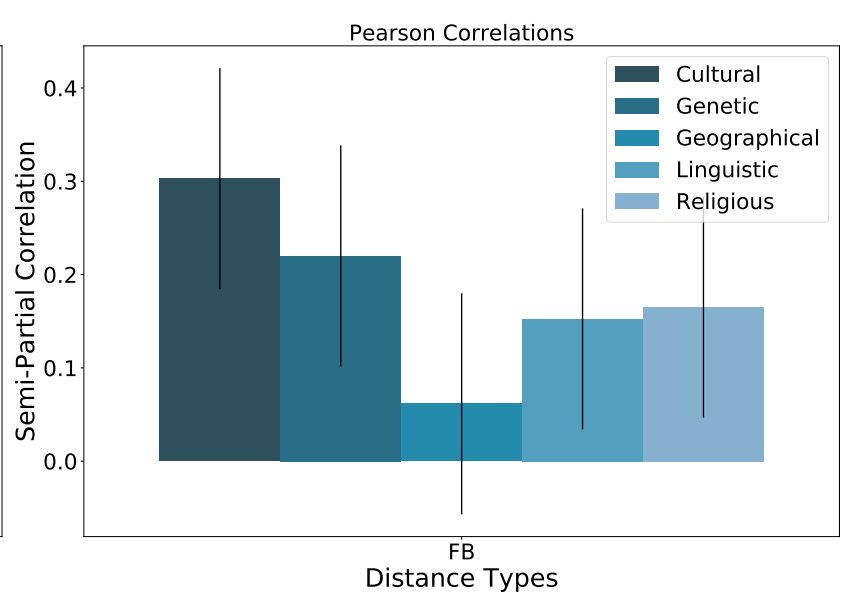

(d) Semi-Partial Correlation (Actual on Residuals)

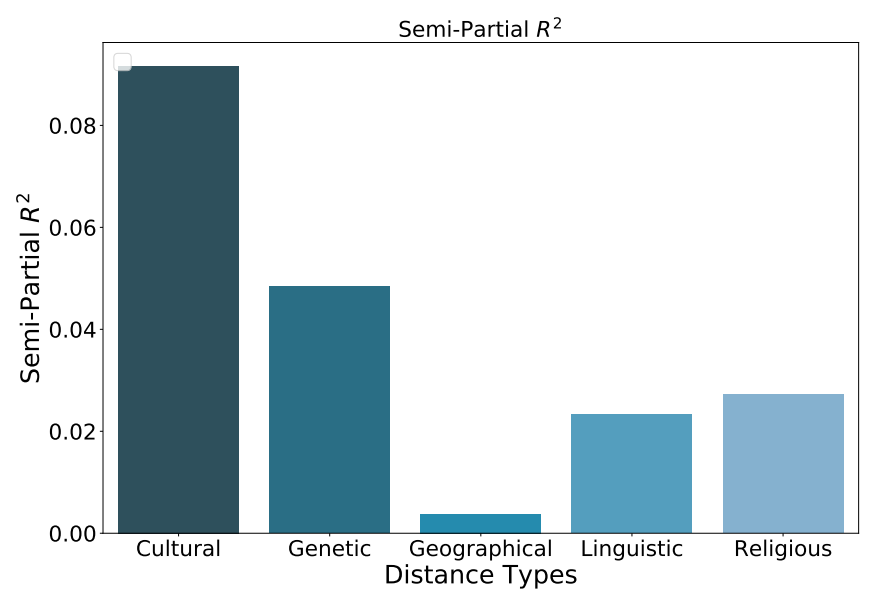

(e) Semi-Partial $R^{2}$

Figure S4: Correlations Between Facebook and Selected Distance Measures, Robustness to Population Size and Facebook Penetration. This figure shows the same information as Figure S1 and S2, with one difference: it does not include the countries with a population of less than 300,000 and a Facebook penetration of less than 5\%. For that different sample, Panel (a) corresponds to Panel (a) of Figure S2, and Panels (b) through (e) correspond to Panels (a) through (d) of Figure S1. 


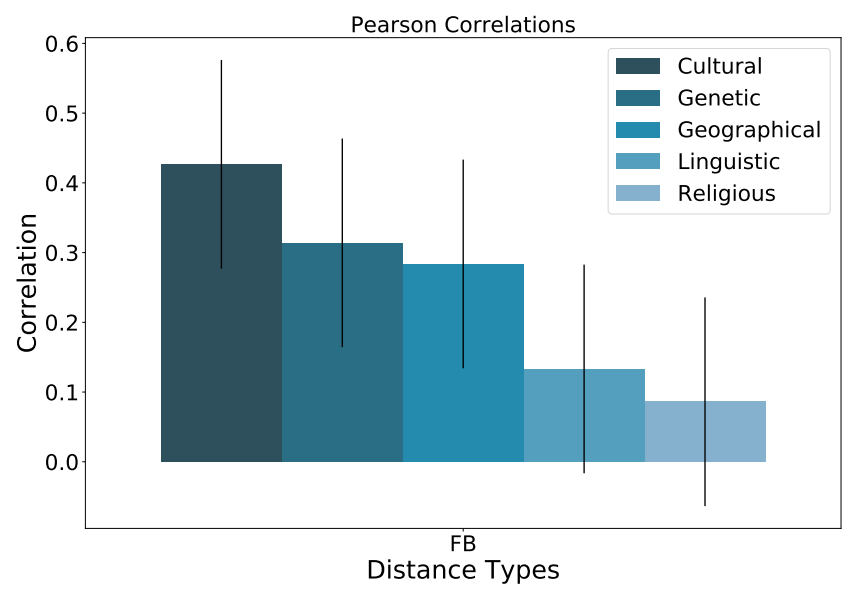

(a) Full Sample

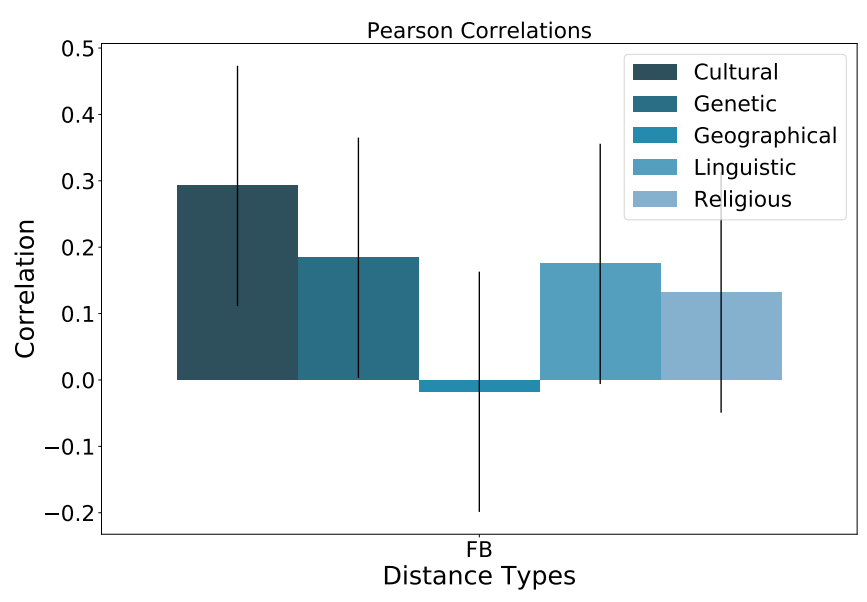

(c) Partial Correlation (Residuals on Residuals)

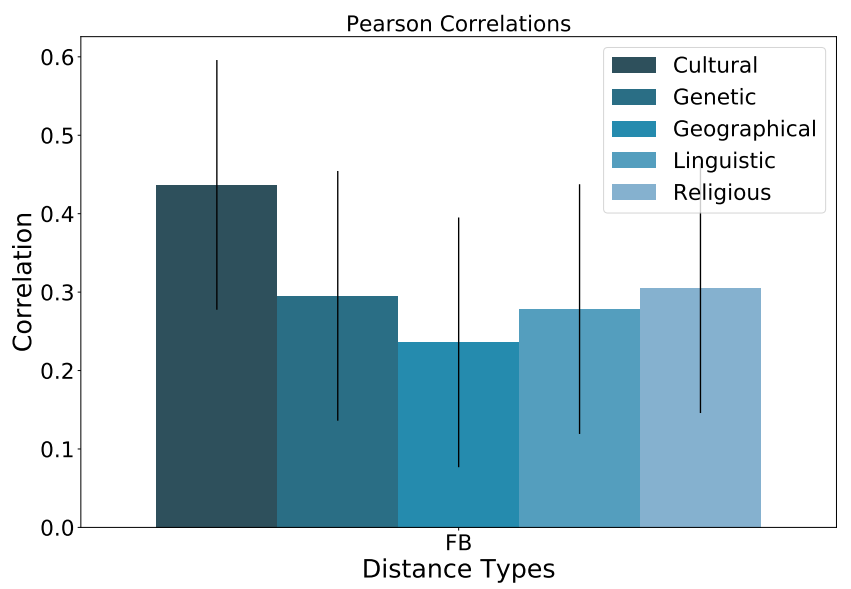

(b) Common Sample

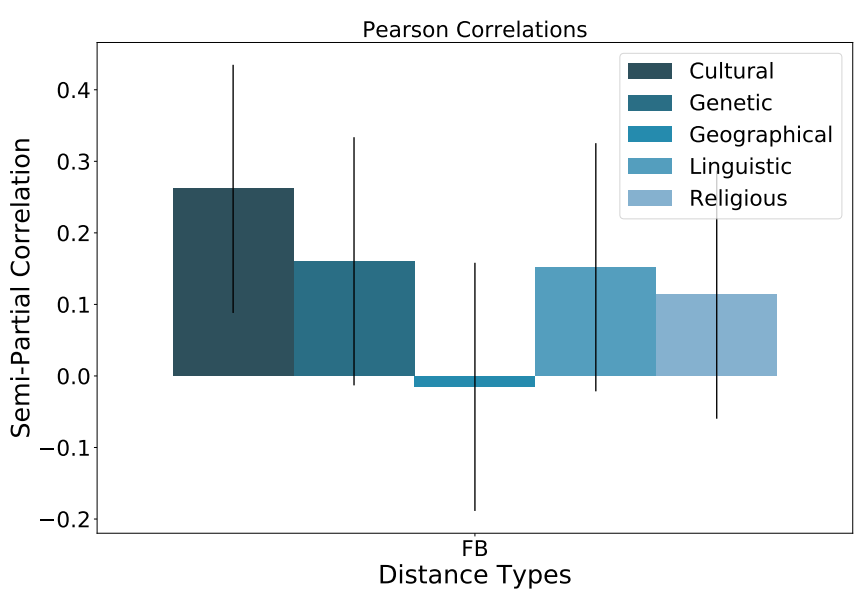

(d) Semi-Partial Correlation (Actual on Residuals)

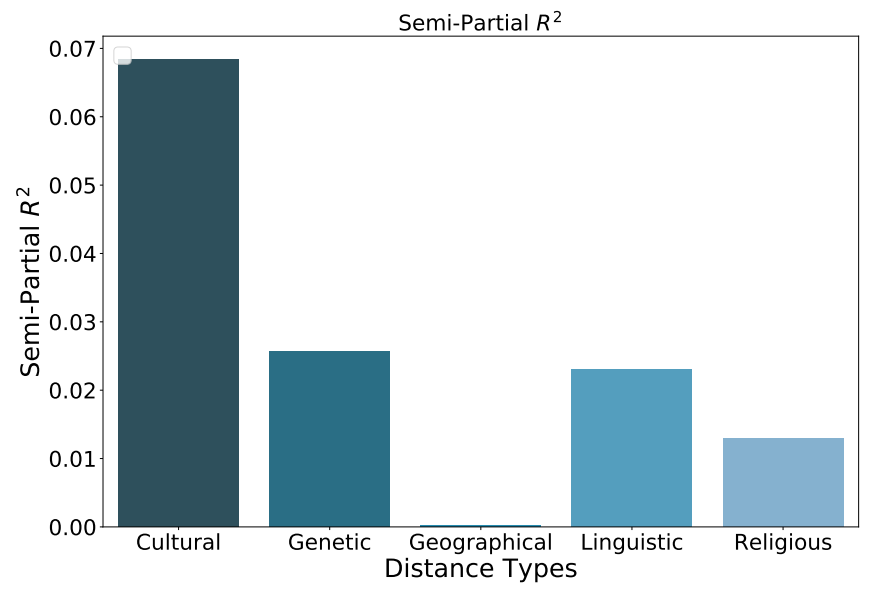

(e) Semi-Partial $R^{2}$

Figure S5: Correlations Between Facebook and Selected Distance Measures, Robustness to Representativity (Gender Difference above Median). This figure shows the same information as Figure S1 and S2, with one difference: using the common sample, it only retains the countries where the difference in gender composition of Facebook users and the actual population is above the median. Hence, it focuses on the subset of countries where Facebook users are least representative of the actual population in terms of gender. For that sample, Panel (a) corresponds to Panel (a) of Figure S2, and Panels (b) through (e) correspond to Panels (a) through (d) of Figure S1. 


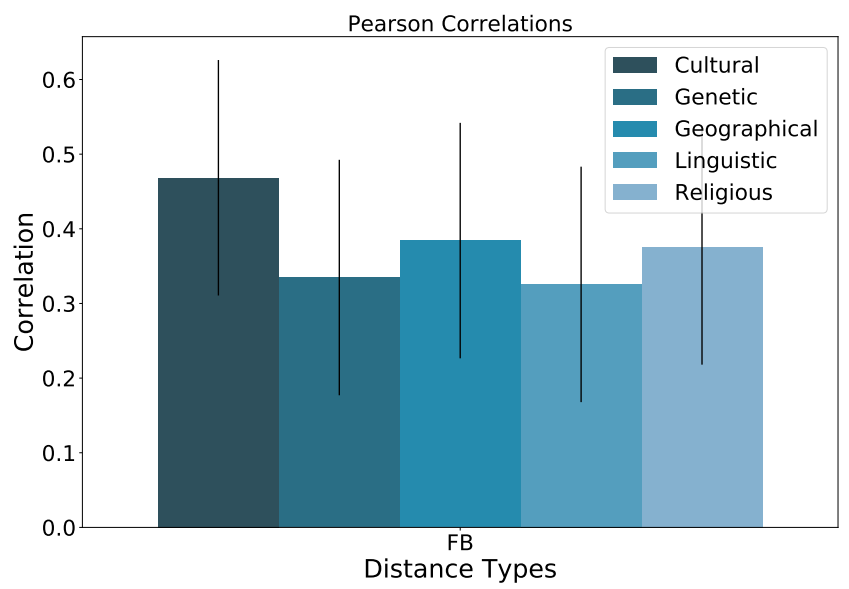

(a) Full Sample

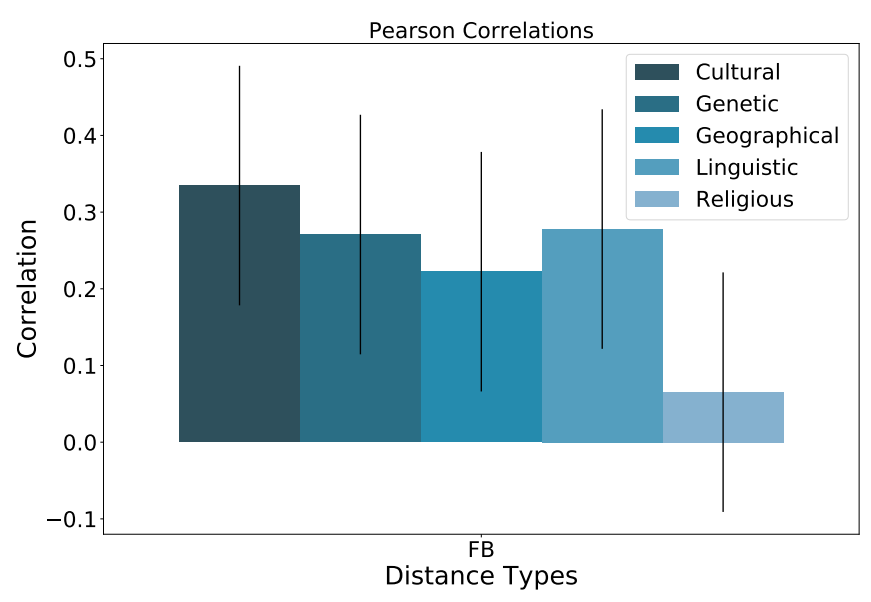

(c) Partial Correlation (Residuals on Residuals)

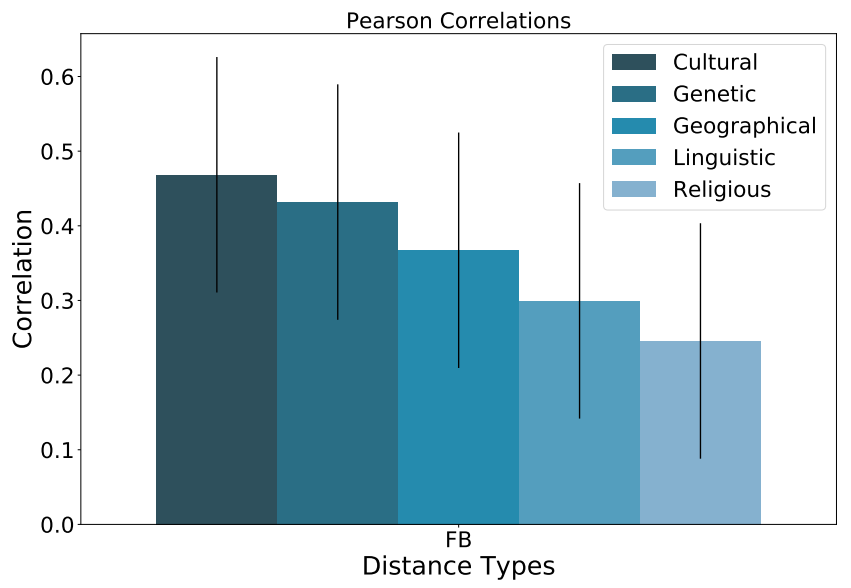

(b) Common Sample

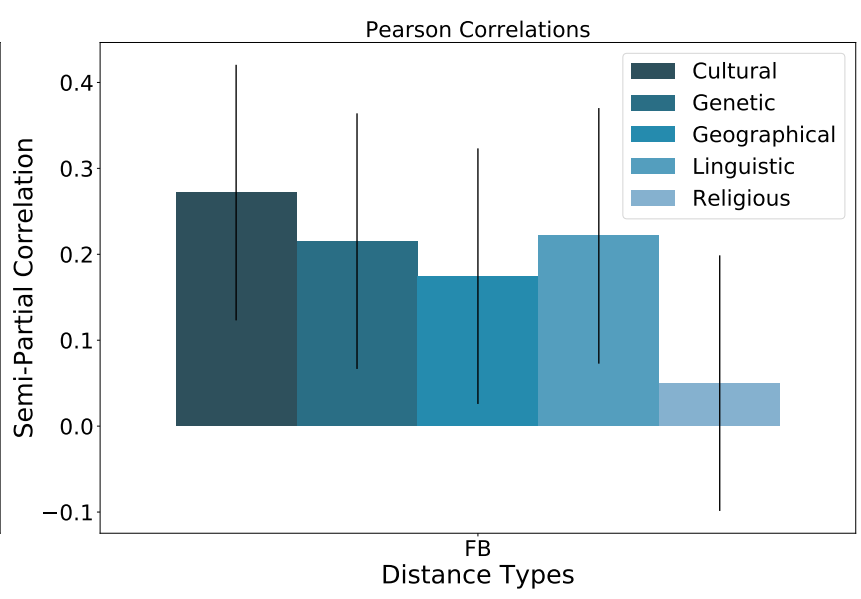

(d) Semi-Partial Correlation (Actual on Residuals)

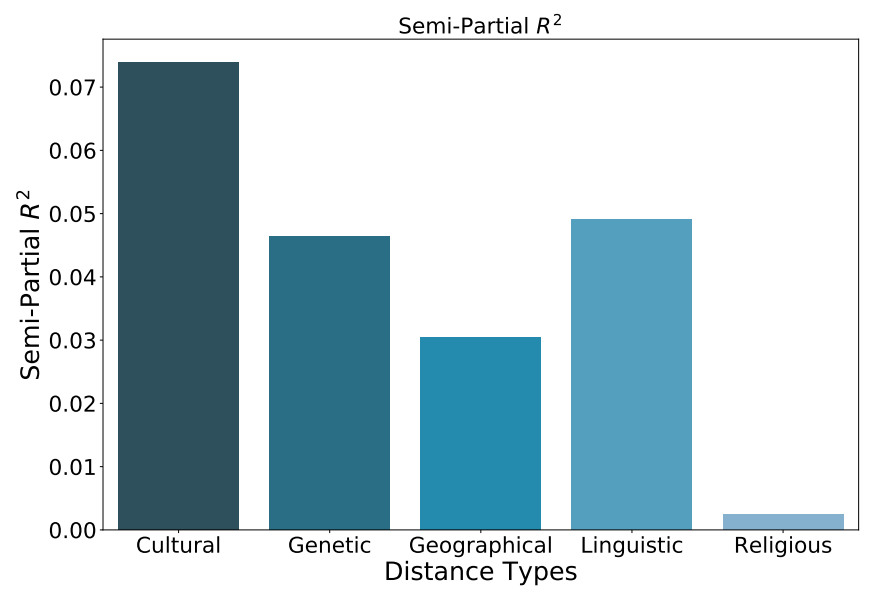

(e) Semi-Partial $R^{2}$

Figure S6: Correlations Between Facebook and Selected Distance Measures, Robustness to Representativity (Gender Difference below Median). This figure shows the same information as Figure S1 and S2, with one difference: using the common sample, it only retains the countries where the difference in gender composition of Facebook users and the actual population is below the median. Hence, it focuses on the subset of countries where Facebook users are most representative of the actual population in terms of gender. For that sample, Panel (a) corresponds to Panel (a) of Figure S2, and Panels (b) through (e) correspond to Panels (a) through (d) of Figure S1. 


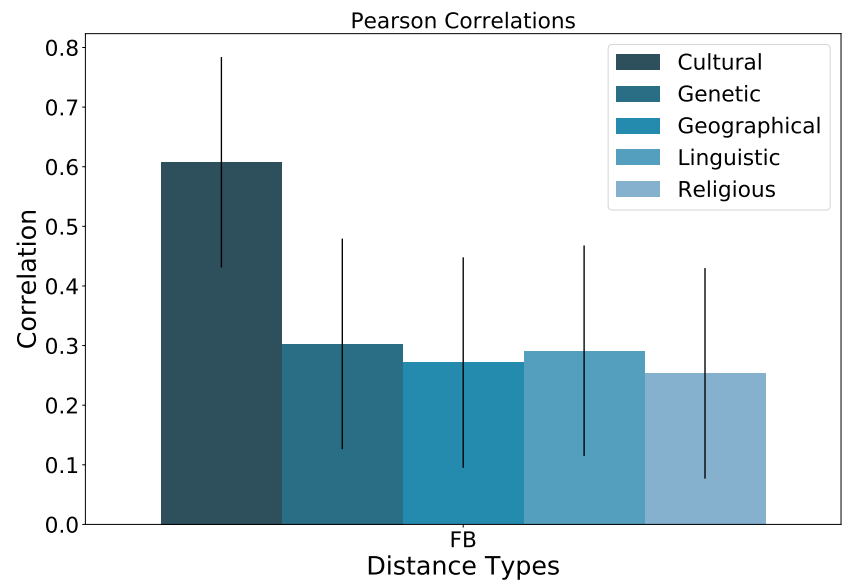

(a) Full Sample

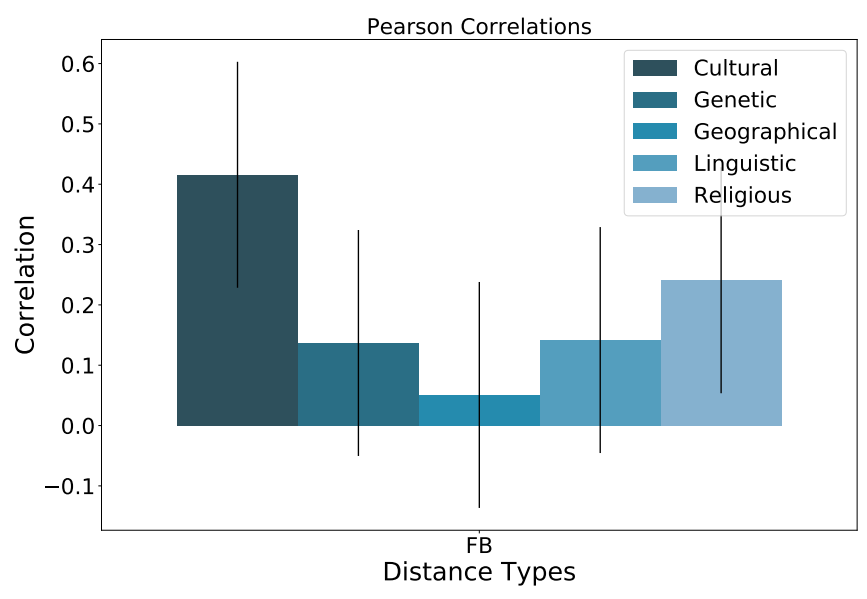

(c) Partial Correlation (Residuals on Residuals)

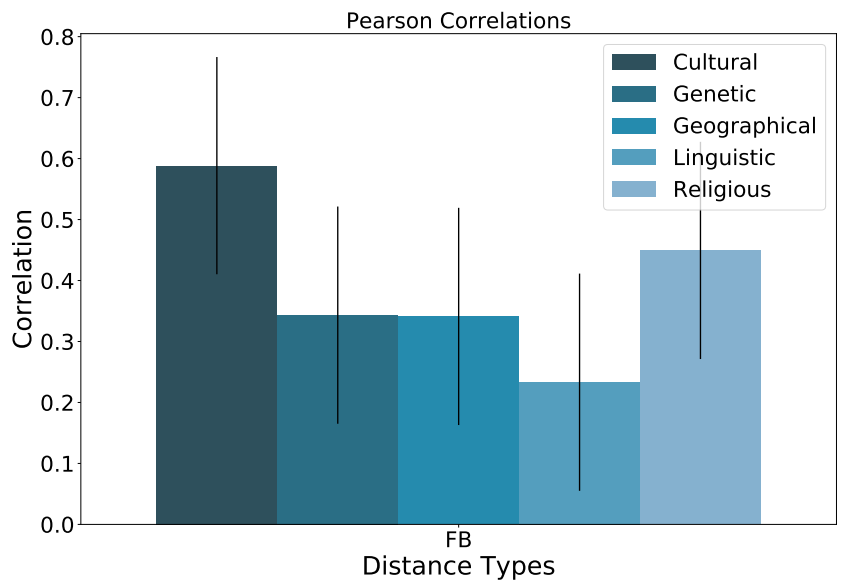

(b) Common Sample

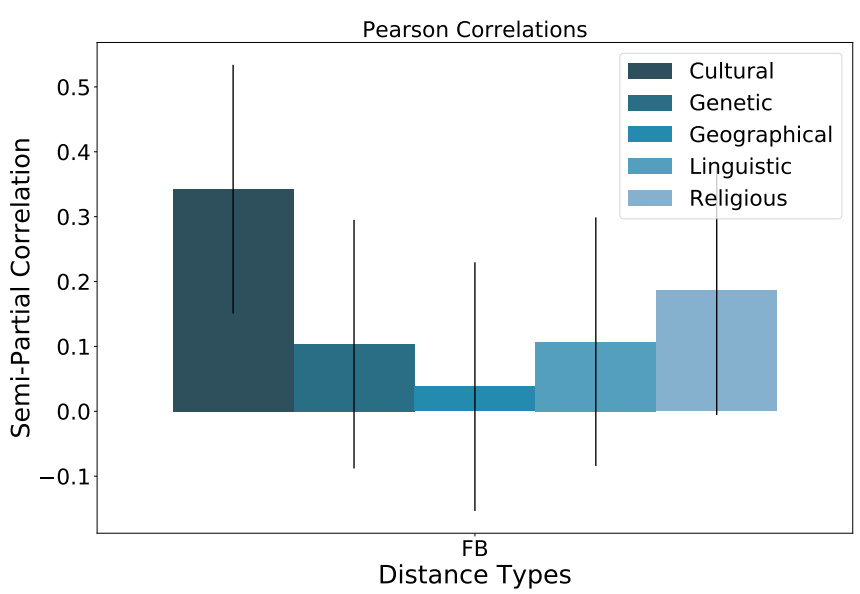

(d) Semi-Partial Correlation (Actual on Residuals)

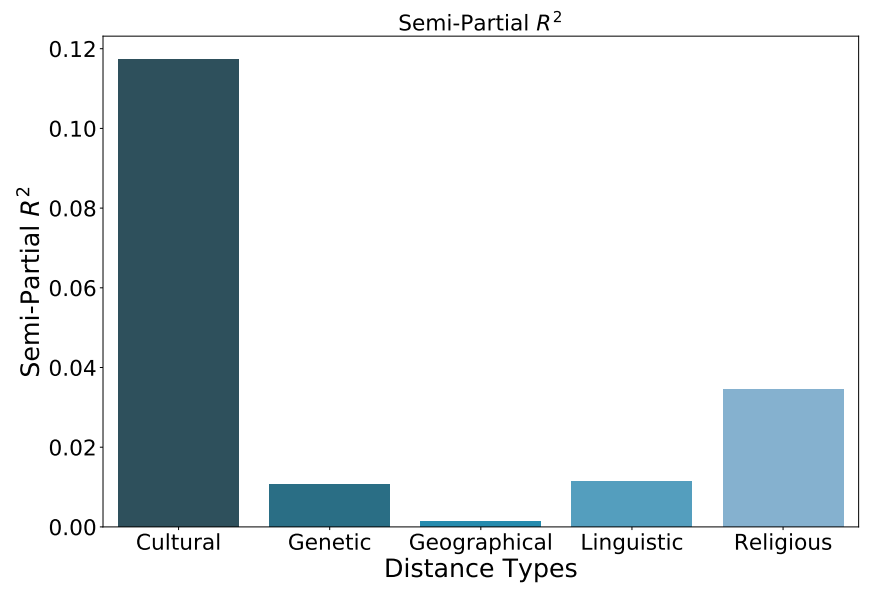

(e) Semi-Partial $R^{2}$

Figure S7: Correlations Between Facebook and Selected Distance Measures, Robustness to Representativity (Age Difference Above Median). This figure shows the same information as Figure S1 and S2, with one difference: using the common sample, it only retains the countries where the difference in age composition of Facebook users and the actual population is above the median. Hence, it focuses on the subset of countries where Facebook users are least representative of the actual population in terms of age. For that sample, Panel (a) corresponds to Panel (a) of Figure S2, and Panels (b) through (e) correspond to Panels (a) through (d) of Figure S1. 


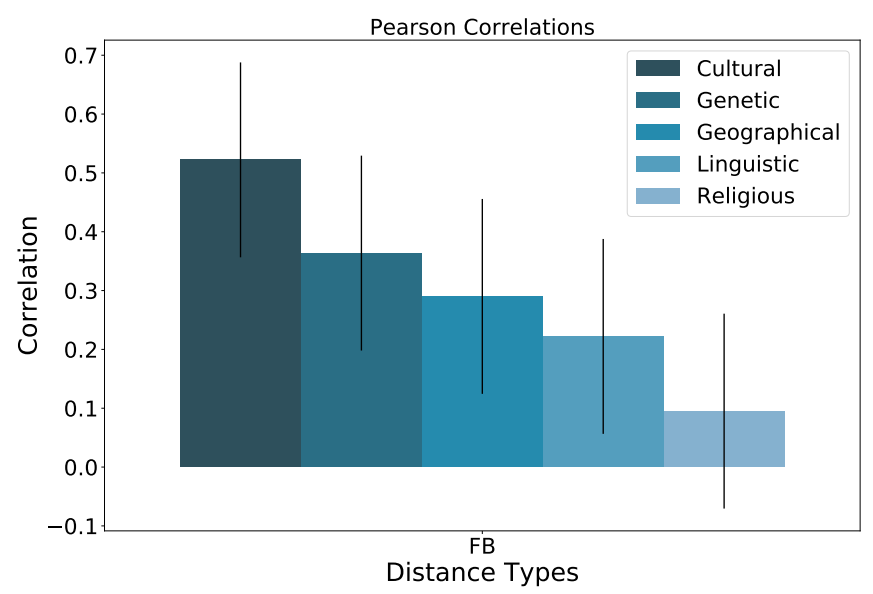

(a) Full Sample

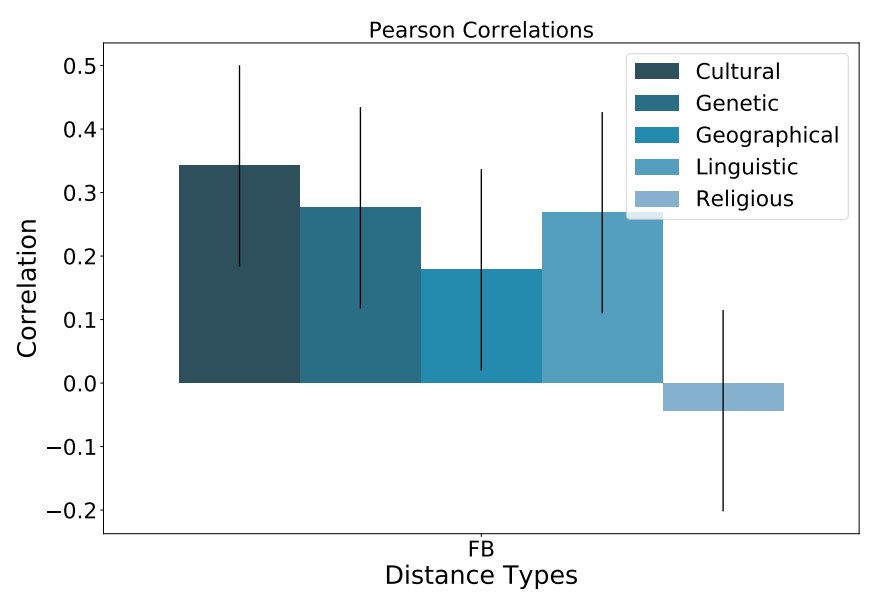

(c) Partial Correlation (Residuals on Residuals)

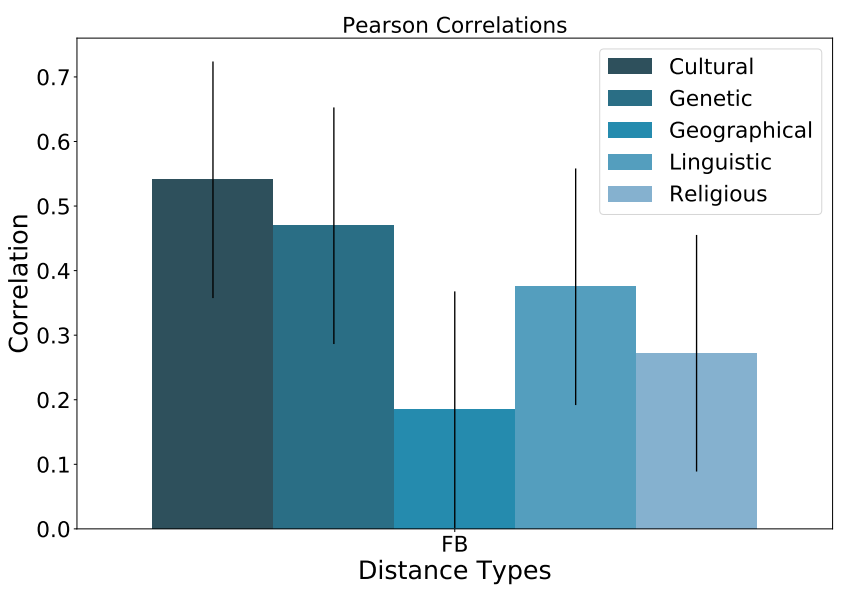

(b) Common Sample

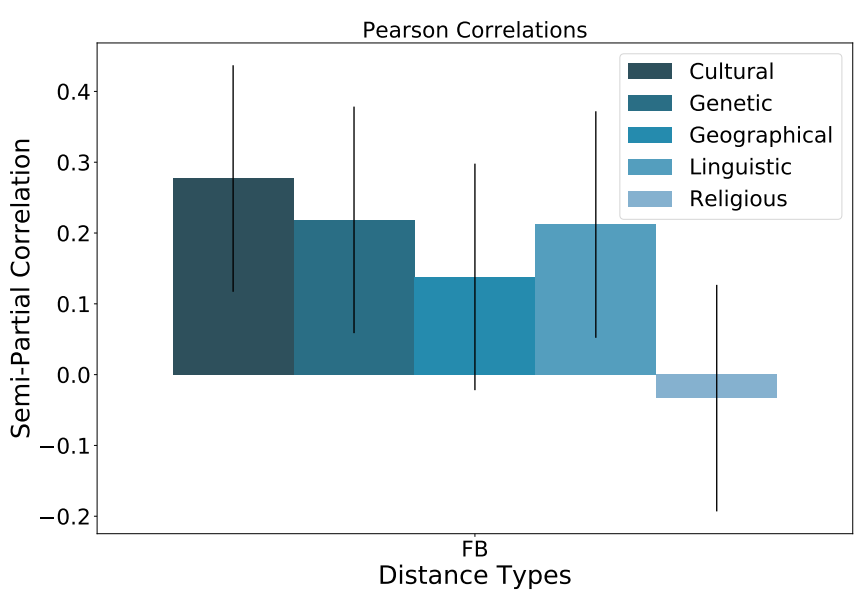

(d) Semi-Partial Correlation (Actual on Residuals)

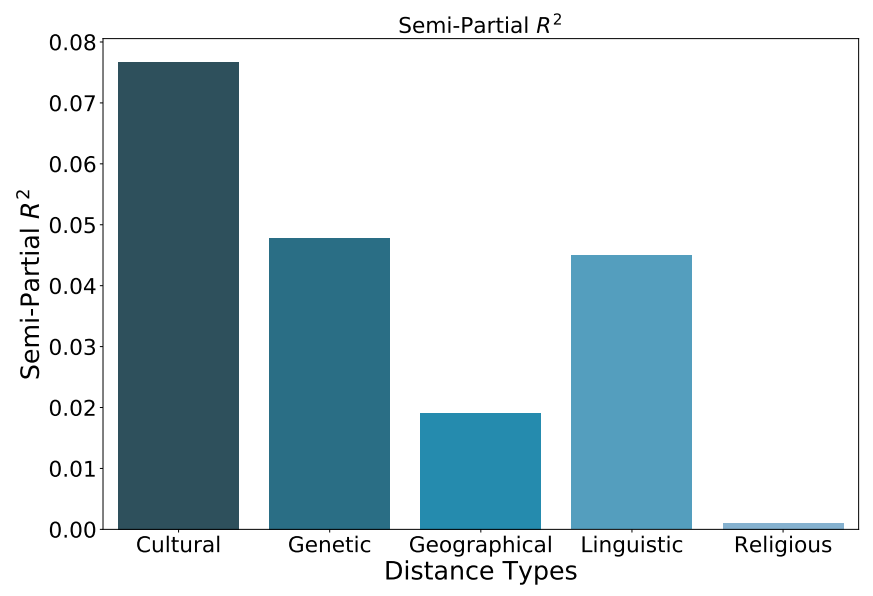

(e) Semi-Partial $R^{2}$

Figure S8: Correlations Between Facebook and Selected Distance Measures, Robustness to Representativity (Age Difference Below Median). This figure shows the same information as Figure S1 and S2, with one difference: using the common sample, it only retains the countries where the difference in age composition of Facebook users and the actual population is below the median. Hence, it focuses on the subset of countries where Facebook users are most representative of the actual population in terms of age. For that sample, Panel (a) corresponds to Panel (a) of Figure S2, and Panels (b) through (e) correspond to Panels (a) through (d) of Figure S1. 


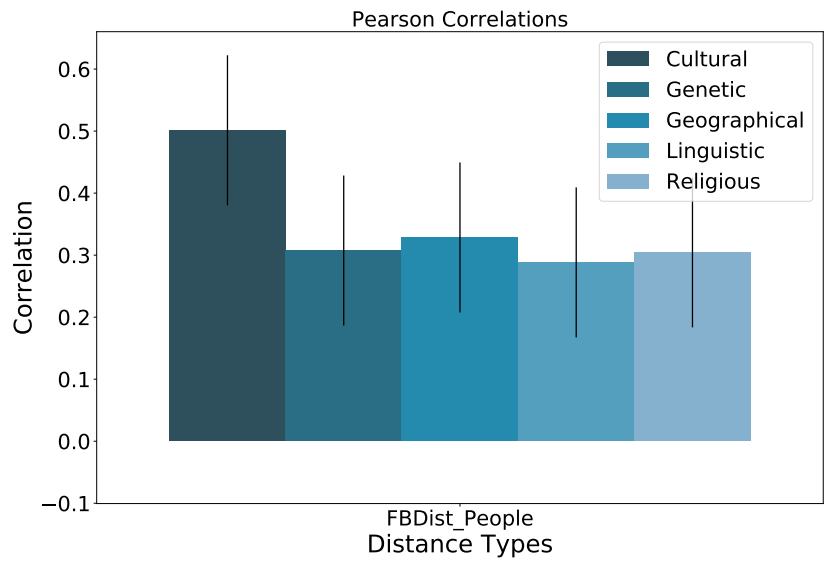

(a) People

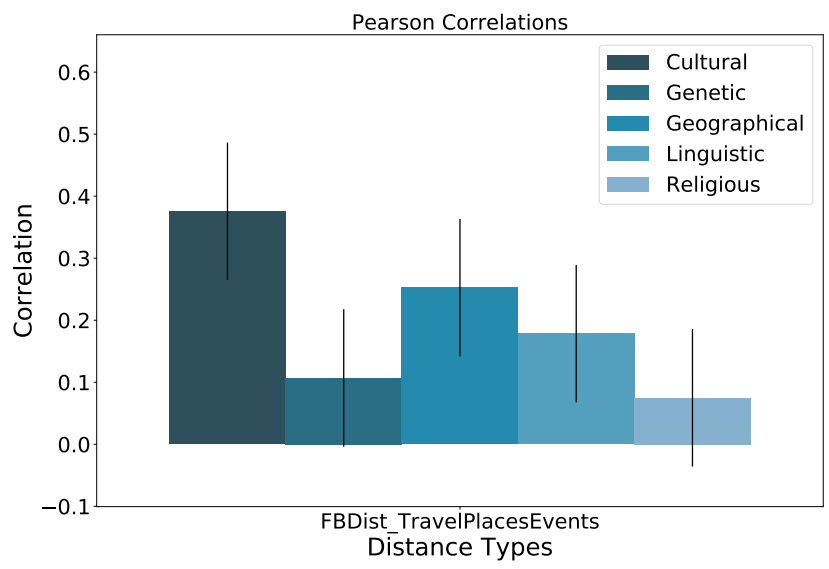

(c) Travel, Places \& Events

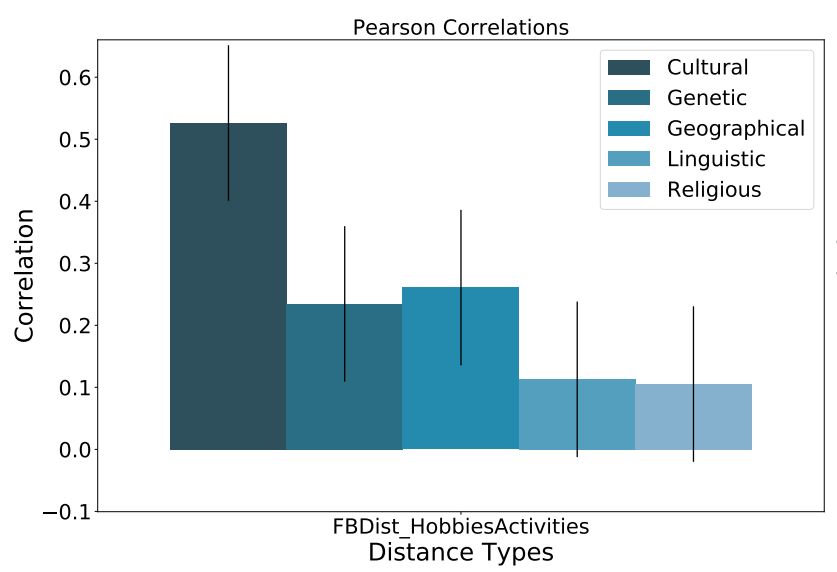

(e) Hobbies \& Activities

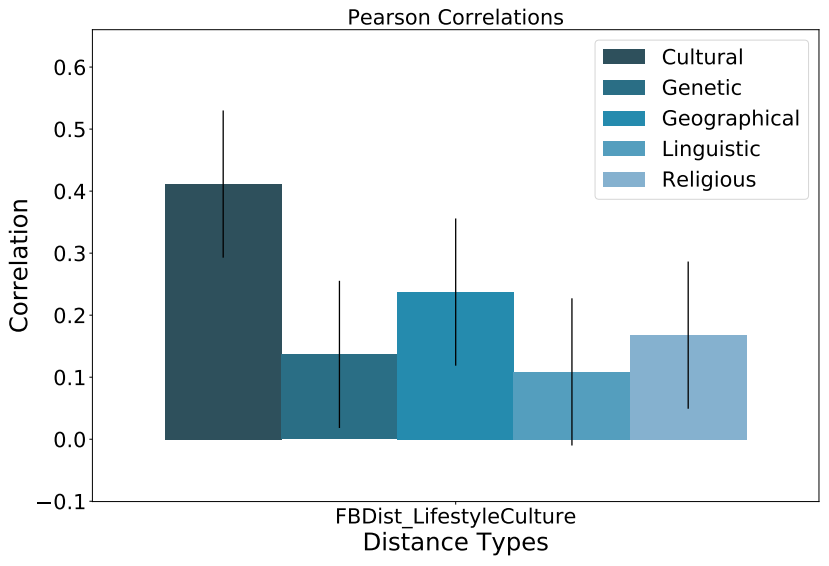

(b) Lifestyle \& Culture

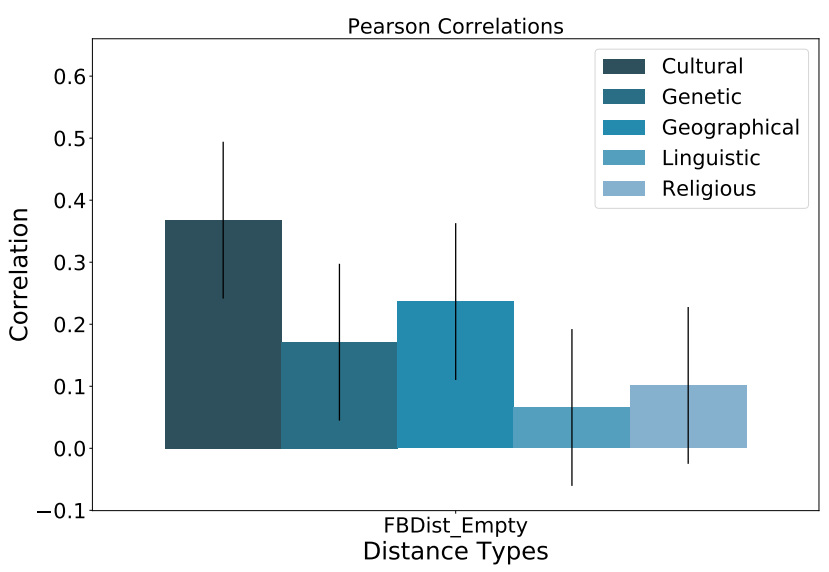

(d) Empty

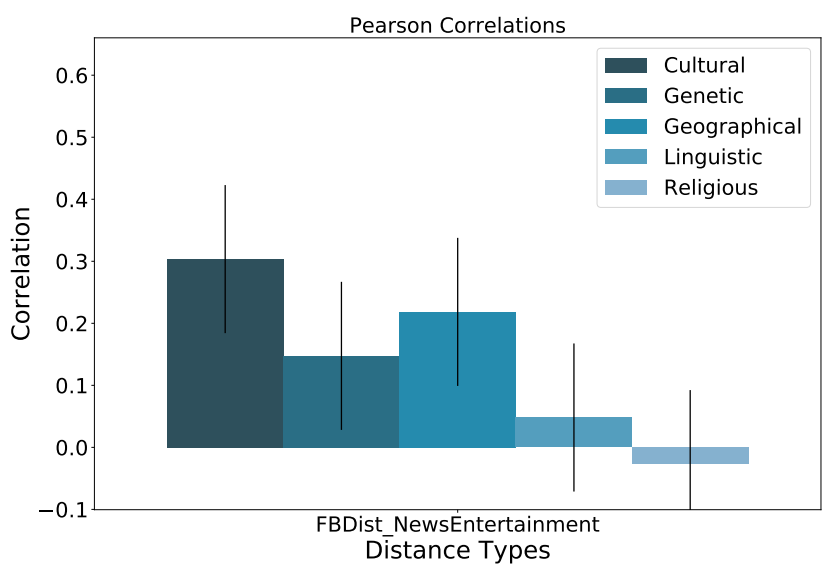

(f) News \& Entertainment

Figure S9: Correlations Between Facebook and Selected Distance Measures, Robustness to Facebook Categories (cont.). Each panel plots the correlations between Facebook distances and each one of the distance measures, not controlling for any other distance. Panels (a) through (n) depict these correlations for each one of the 14 macro-categories of interests: people; lifestyle and culture; travel, places and events; empty; hobbies and activities; news and entertainment; shopping and fashion; business and industry; food and drink; sports and outdoors; education; technology; fitness and wellness; and family and relationships. Panel (o) depicts these correlations for all interests that are not marked as local businesses. 


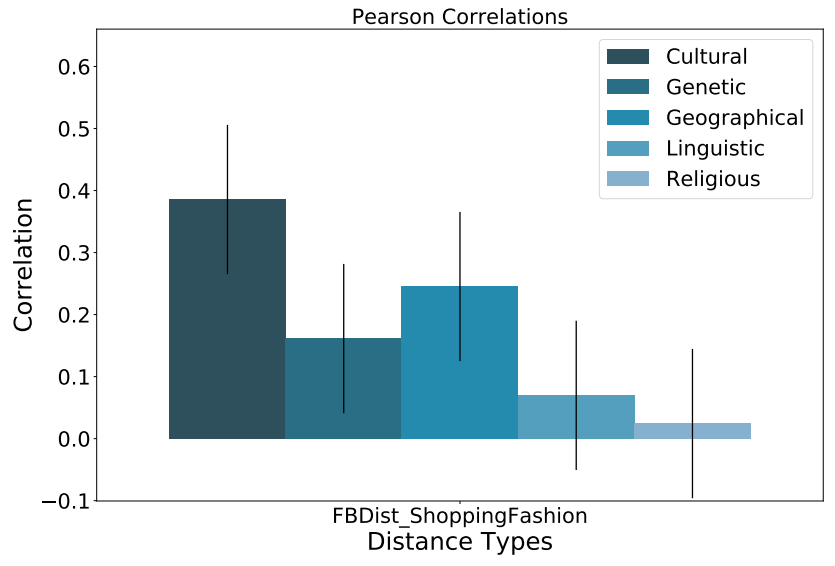

(a) Shopping \& Fashion

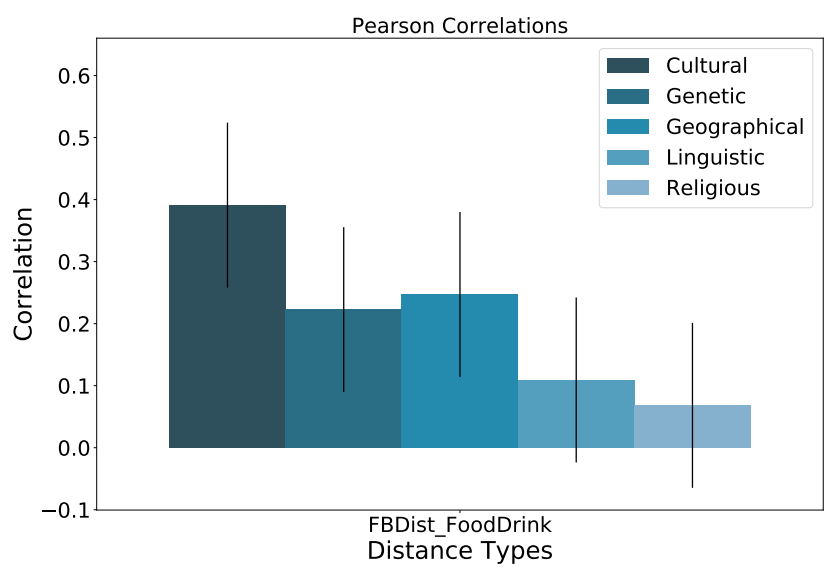

(c) Food \& Drink

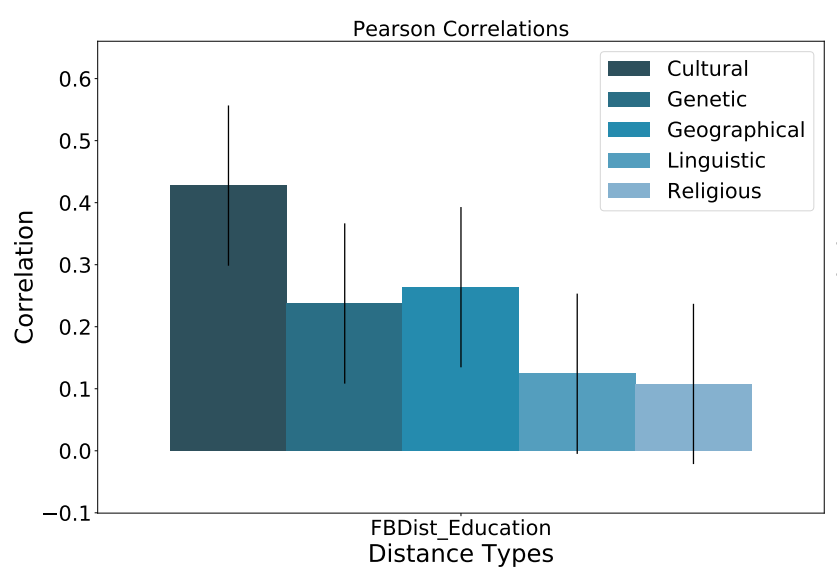

(e) Education

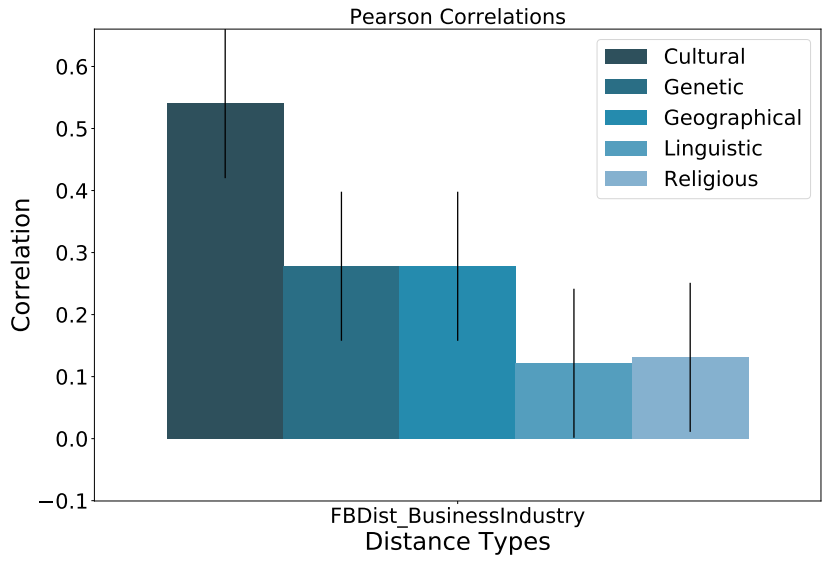

(b) Business \& Industry

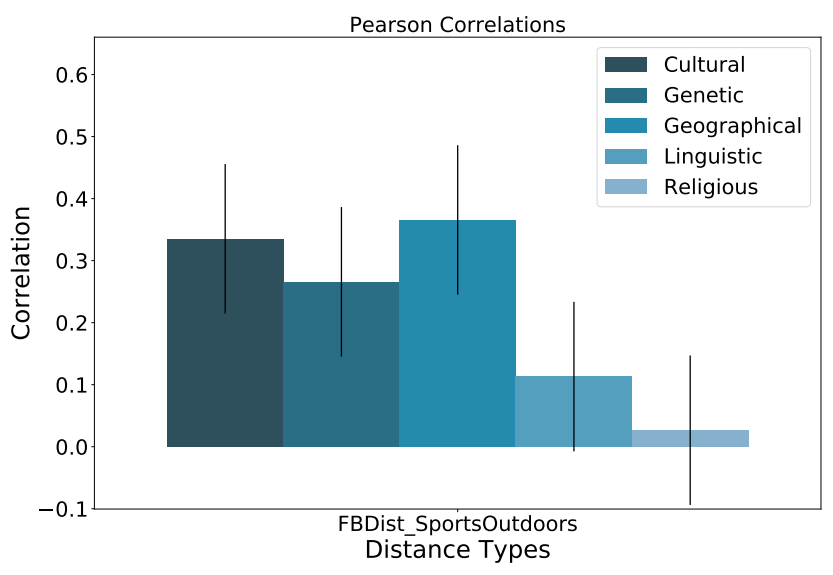

(d) Sports \& Outdoors

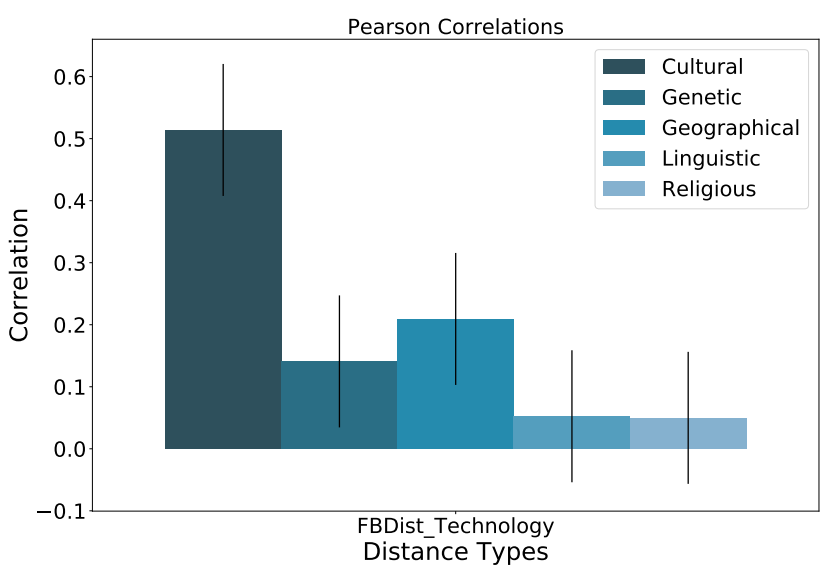

(f) Technology

Figure S9: Correlations Between Facebook and Selected Distance Measures, Robustness to Facebook Categories (cont.). Each panel plots the correlations between Facebook distances and each one of the distance measures, not controlling for any other distance. Panels (a) through ( $n$ ) depict these correlations for each one of the 14 macro-categories of interests: people; lifestyle and culture; travel, places and events; empty; hobbies and activities; news and entertainment; shopping and fashion; business and industry; food and drink; sports and outdoors; education; technology; fitness and wellness; and family and relationships. Panel (o) depicts these correlations for all interests that are not marked as local businesses. 


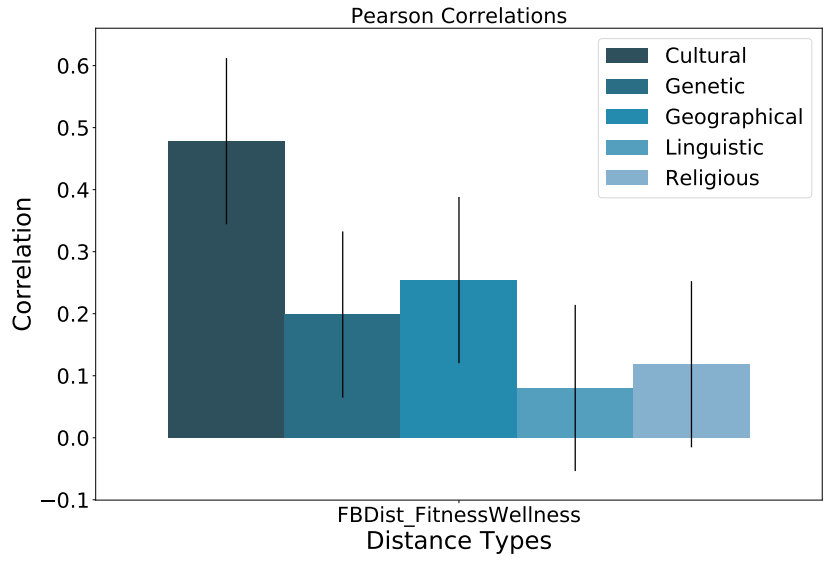

(g) Fitness \& Wellness

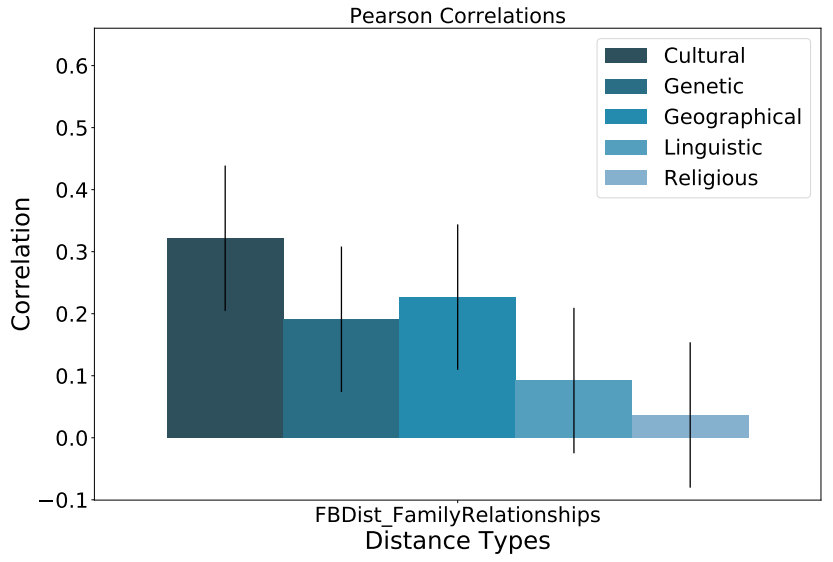

(h) Family \& Relationships

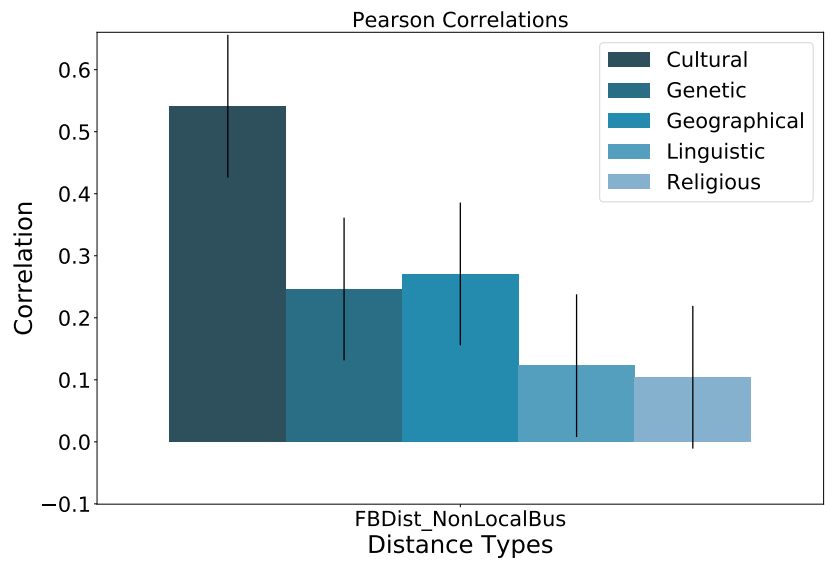

(i) Non Local Business

Figure S9: Correlations Between Facebook and Selected Distance Measures, Robustness to Facebook Categories (cont.). Each panel plots the correlations between Facebook distances and each one of the distance measures, not controlling for any other distance. Panels (a) through (n) depict these correlations for each one of the 14 macro-categories of interests: people; lifestyle and culture; travel, places and events; empty; hobbies and activities; news and entertainment; shopping and fashion; business and industry; food and drink; sports and outdoors; education; technology; fitness and wellness; and family and relationships. Panel (o) depicts these correlations for all interests that are not marked as local businesses. 


\section{Robustness to other distance measures}

Figure S10 reports our main results based on normalized Euclidean distances, rather than cosine distance. Since the former is a simple transformation of the latter, our findings are unchanged.

\section{Alternative methods for principal component analysis}

As mentioned in the Methods, principal component analysis can be done on the covariance matrix or on the correlation matrix. When using the correlation matrix, we are standardizing the population share for each one of the interests or questions, whereas when using the covariance matrix, we are not. In the context of our data it is not obvious whether one should standardize or not. Standardization is often done to make the variables scale-independent. Since our variables are population shares, they are already measured on the same scale, from 0 to 1 . Of course standardization still matters, since there are interests or questions with very low average shares, and others with very high average shares. Standardizing puts equal weight on all interests, whereas not standardizing puts greater weight on interests with larger average shares.

Figure $2 \mathrm{C}$ in the main text is based on the covariance matrix, whereas Figure S11 shows results for both the correlation and the covariance matrix. In particular, Figure S10 plots the share of the overall variance in questions and interests that is explained by principal components as a function of their number: the left panel is based on the correlation matrix (i.e., standardized population shares), whereas the right panel is based on the covariance matrix (i.e., non-standardized population shares). Focusing on the standardized shares, the first ten principal components of FB explain slightly more than $40 \%$ of the overall variance in FB interests, whereas the first ten principal components of WVS explain slightly less than $70 \%$ of the variance in WVS questions. When using non-standardized shares, the share of the variance that is explained by the first ten principal components increases by about ten percentage points, to slightly less than $60 \%$ in the case of FB and to around $80 \%$ in the case of WVS. From this we conclude that FB captures more dimensions of culture than the WVS.

\section{Cultural center of the world}

In this subsection we explore which country is the cultural center of the world. Suppose the entire world population needs to meet in a particular country. We will refer to the country that minimizes the average Facebook distance traveled by the world population as the cultural center of the world. To determine this country, compute for each country $k$, the average distance traveled by the world population to meet in country $k$ :

$$
\cos \operatorname{dist}(k, W)=\sum_{l} s_{l} \cos \operatorname{dist}(l, k)
$$

When using all 225 countries, Table S1 shows that the cultural center of the world is South Africa, probably the only country in the world with a large African, European and Asian population. Other countries in the top ten include some of the Gulf countries with large immigrant populations (44\% in Oman, 37\% in Saudi Arabia). If we limit ourselves to the sample of 161 countries with more than 300,000 people and a Facebook penetration above $5 \%$, the cultural center of the world is India, although South Africa continues to be in the top-3. If instead of taking the actual population shares as the shares in (4) we consider the Facebook user shares, the cultural center of the world for the same sample of 161 countries becomes Switzerland. The cultural center based on 


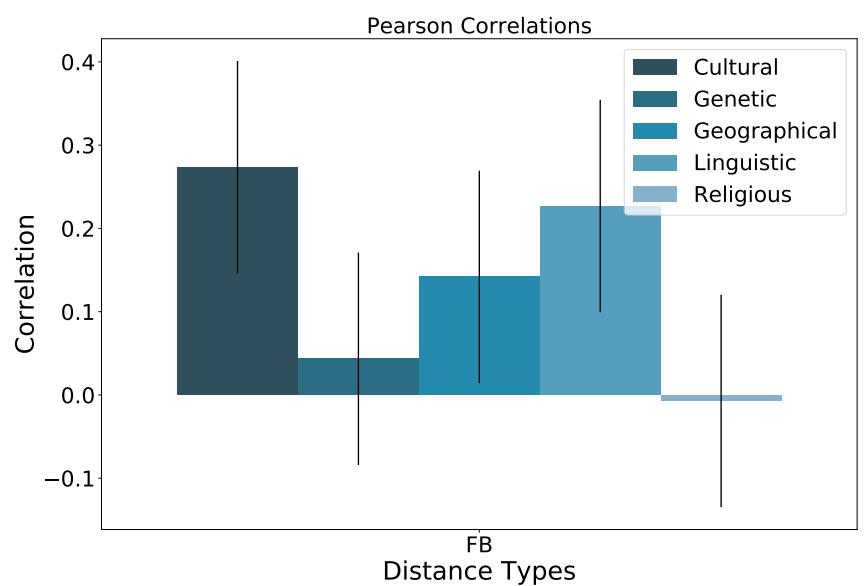

(j) Full Sample

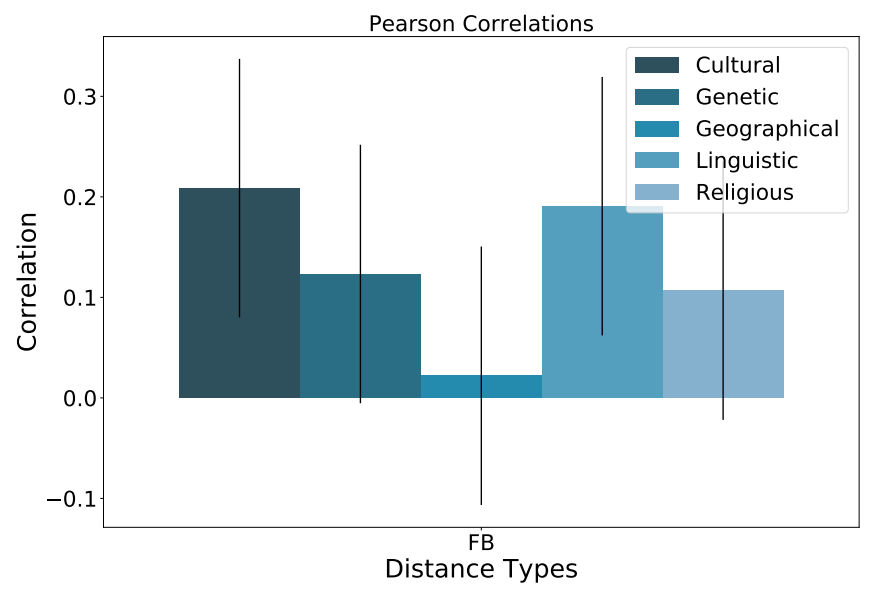

(1) Partial Correlation (Residuals on Residuals)

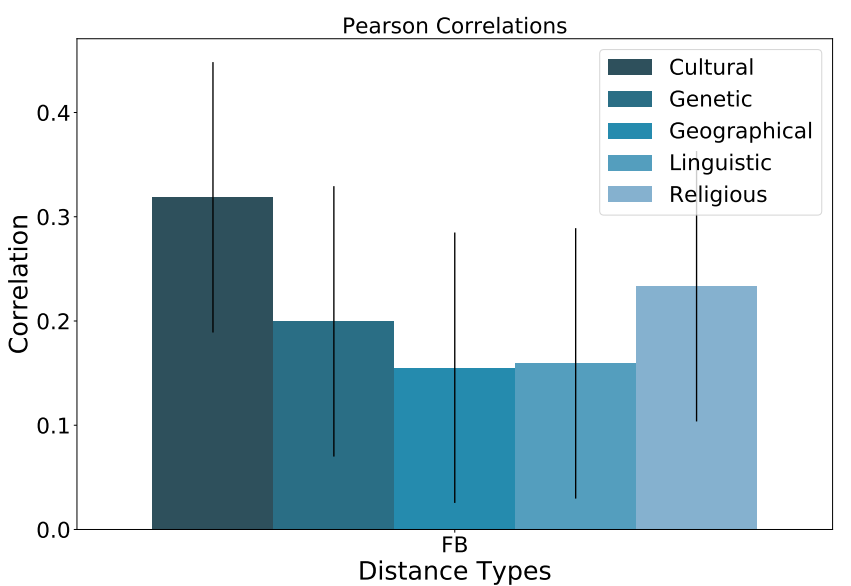

(k) Common Sample

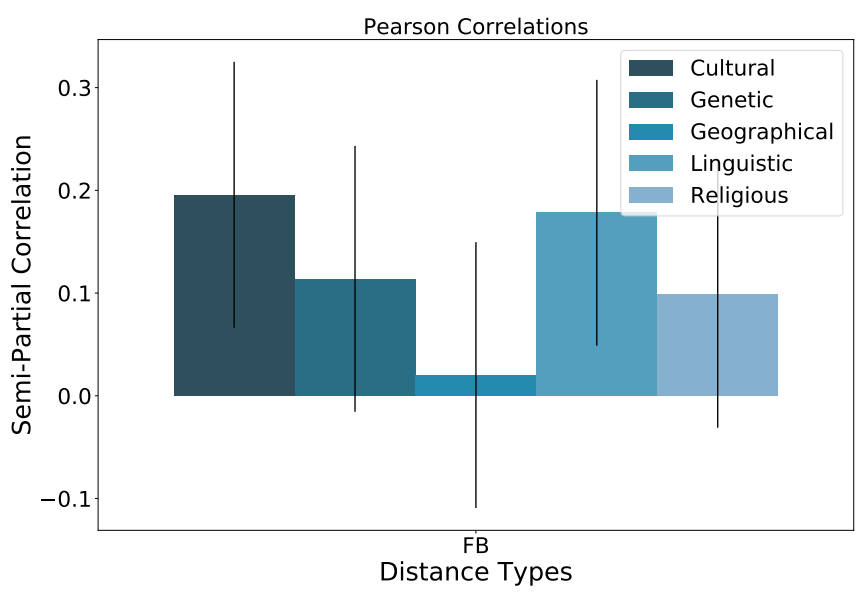

(m) Semi-Partial Correlation (Actual on Residuals)

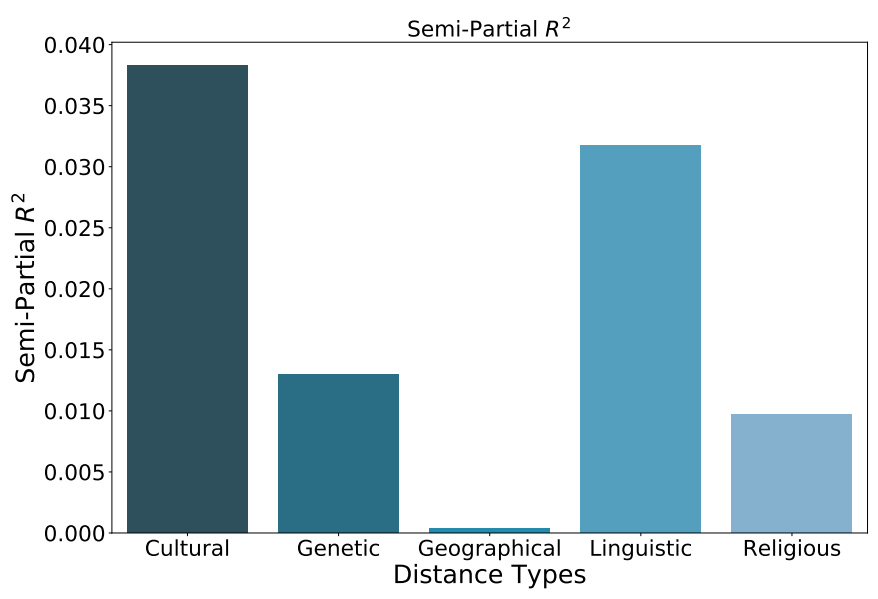

(n) Semi-Partial $R^{2}$

Figure S10: Correlations Between Facebook and Selected Distance Measures, Euclidean Distances. This figure shows the same information as Figure S1 and S2, with one difference: it uses Euclidean, rather than cosine distances. Using Euclidean distances, Panel (a) corresponds to Panel (a) of Figure S2, and Panels (b) through (e) correspond to Panels (a) through (d) of Figure S1. 


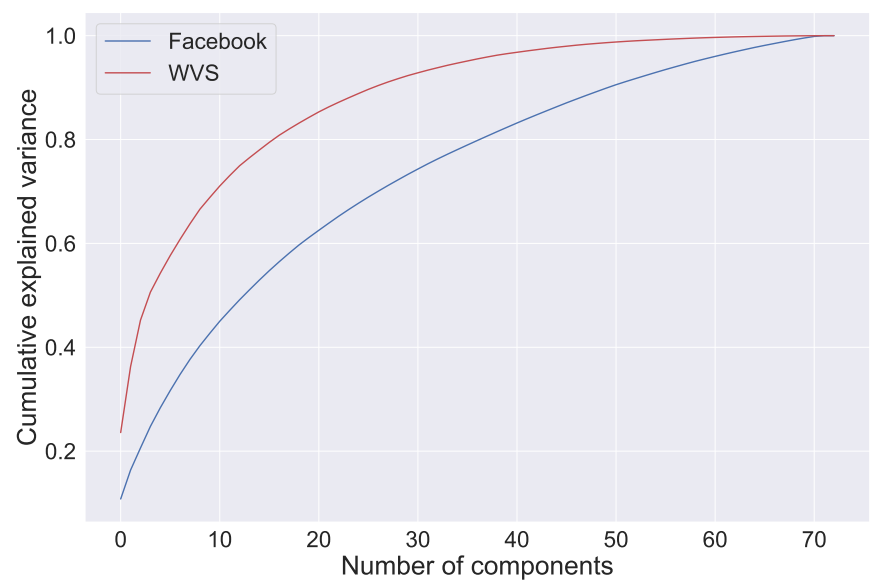

(a) Variance explained by PC (correlation)

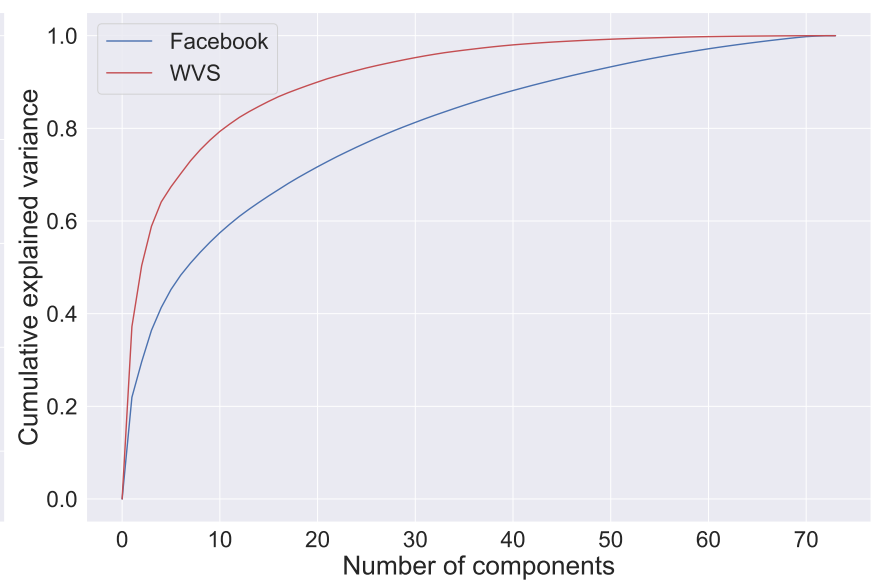

(b) Variance explained by PC (covariance)

Figure S11: Variance explained by principal components. This figure shows the number of independent underlying factors behind the variation in our Facebook measure and compares it to the traditional values-based measure. Specifically, it shows the cumulative share of total variation in our Facebook measure and the values-based measure explained by principal components. Panel (a) plots explained variance after standardizing, i.e. using the correlation matrix. Panel (b) plots explained variance without standardizing, i.e. using the covariance matrix.

Facebook users benefits either countries with large Facebook populations (such as the US) or countries that are culturally close to the bulk of world Facebook users (such as Switzerland or Belgium).

One advantage of using Facebook data to determine the cultural center of the world is its global coverage. For example, standard values surveys often undersample certain regions of the world. Needless to say, this introduces important distortions.

1. Top-10, weighted by actual populations, sample of 225 countries:

South Africa, India, China, Oman, Russia, Benin, France, Tajikistan, Central African Republic, Saudi Arabia

2. Top-10, weighted by actual populations, sample of 161 countries:

India, Oman, South Africa, Saudi Arabia, Germany, Qatar, France, Kyrgyzstan, Bahrain, Switzerland

3. Top-10, weighted by Facebook users, sample of 225 countries:

South Africa, France, Switzerland, Canada, US, Belgium, Germany, Great Britain, Guyana, Panama

4. Top-10, weighted by Facebook users, sample of 161 countries:

Switzerland, France, Germany, Belgium, Canada, South Africa, Great Britain, Panama, US, Guyana

Table S1: Cultural center of the world. This table reports the top-10 countries that minimize the average distance the world population (or the world Facebook users) would need to travel if they were to meet in the same location. Calculations are based on (4), and differ depending on samples and weights. The sample can either be all 225 countries for which Facebook data are available (cases 1 and 3) or the subsample of 161 countries with a population of more than 300,000 and a Facebook penetration of more than $5 \%$ (cases 2 and 4). The distance can either be weighted by actual populations (case 1 and 2) or by Facebook users (cases 3 and 4). 


\section{Cultural sister regions}

In this subsection we identify for each subnational region of ten countries the "sister region", defined as the closest foreign region according to Facebook distances. Table S2 shows the results for the subnational regions of ten countries: United States (US), Spain (ES), France (FR), Germany (DE), Italy (IT), Portugal (PT), Great Britain (GB), Belgium (BE), Netherlands (NL) and Ireland (IE). For each subnational region, the cultural sister has to be in one of the other nine countries.

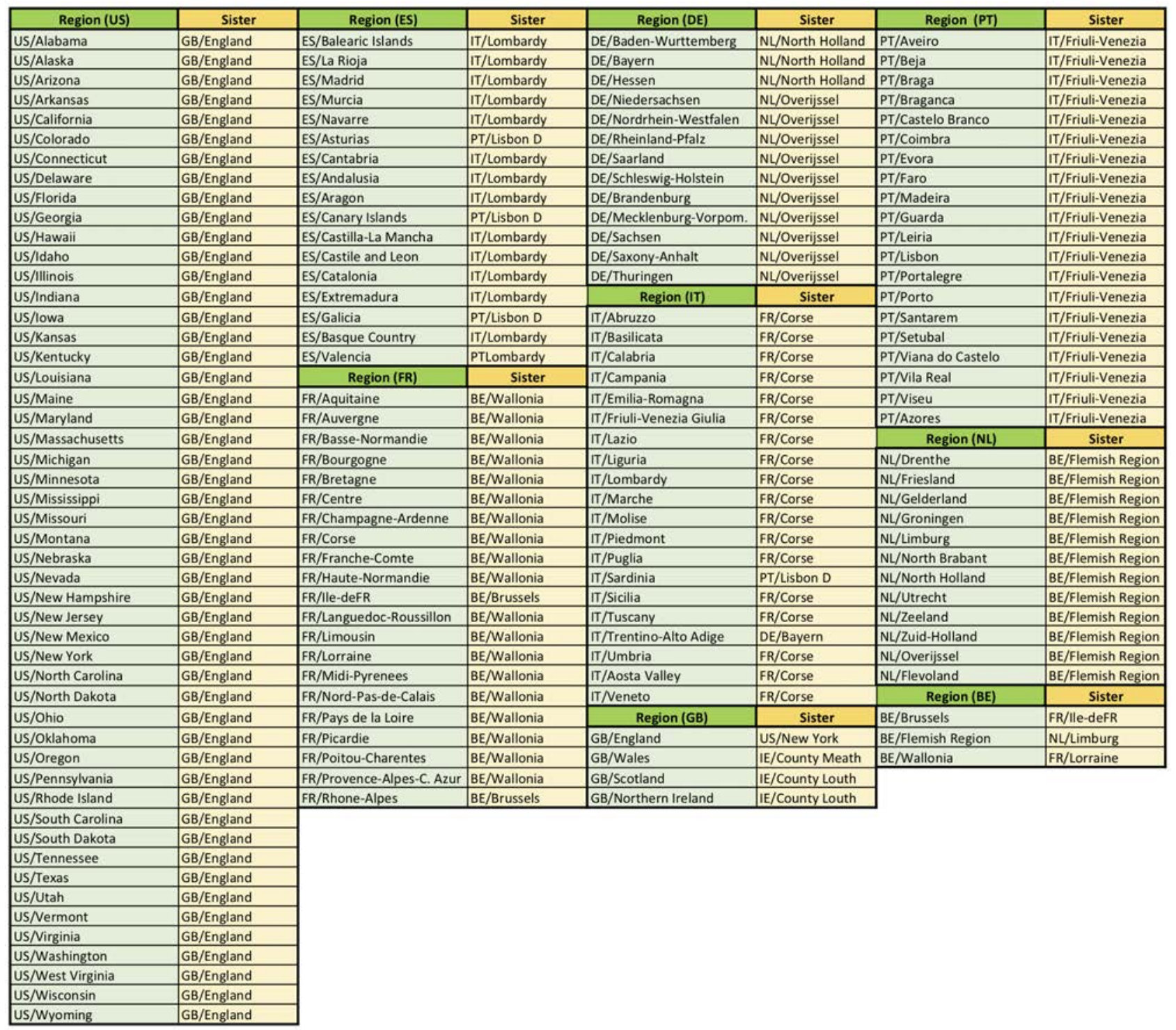

Table S2: “Sister region” for each region in a sample of ten countries: the United States (US), Spain (ES), France $(F R)$, Germany (DE), Italy (IT), Portugal (PT), Great Britain (GB), Belgium (BE), Netherlands (NL) and Ireland (IE). A sister region is defined as the foreign region in one of the other nine countries that is closest according to Facebook distances.

Several results stand out. First, the sister regions tend to be located in the countries that are closest in the dendrogram in Figure 3. For example, the sister regions of U.S. states are in Great Britain, the sister regions of France are in Belgium, and the sister regions of the German Länder are in the Netherlands. Second, subnational regions sometimes have sister regions in different countries, depending on linguistic or geographic proximity. 
For example, most regions in Spain have sister regions in Italy. However, Galicia, a region in northwest Spain that speaks a language closely related to Portuguese, has as sister region Lisbon. Likewise, the Dutch-speaking region of Belgium has as sister region Limburg in the southern Netherlands, whereas the French-speaking region of Belgium has as sister region Lorraine in France. As another example, England has as sister region the state of New York, whereas Scotland has as sister region County Louth on the eastern coast of Ireland. Italy also shows some interesting patterns: while most of its regions are paired to Corsica, a French island with an Italian dialect, the region of Trentino-Alto Adige with a large German-speaking population is paired to Bavaria. Third, the urban nature of regions also sometimes matters. For example, the Paris region of Ile-de-France has as sister region Brussels, the capital of Belgium. The same is true for the French region of Rhône-Alpes, home to the second-largest metropolitan area Lyon.

\section{Regional divisiveness and gender divisiveness}

Given their importance, it is interesting to compare regional differences and gender differences. Broadly speaking, our findings suggest that in most developed countries the gender divide is larger than the regional divide, whereas in many developing countries the regional divide continues to be important (Figure 7).

Rather than comparing the average interregional distance with the gender distance, we could also compare all bilateral distances between regions in a given country to the bilateral distance between genders in that same country. Figure S12 displays for each country a kernel density plot of the bilateral distances between regions as well as the bilateral distance between genders. Two observations stand out. First, interregional distances vary widely, both in their variance across countries and in some cases in their variance within countries. Second, in comparison, gender differences are relatively similar across countries.

In some countries, such as France and Germany, the distance between genders is greater than the bilateral distance between any two regions. For many other countries, the picture is more complex. Take, for instance, the United States. There, the gender difference is greater than many, but not all, interstate differences. As a comparison: while the average distance between Texas and California residents is larger than the average distance between men and women in the U.S., the opposite is true for the average distance between Massachusetts and Connecticut residents or between New Mexico and Colorado residents. As another example, consider Kenya. There, the average gender divide is similar to the average regional divide. However, the regional divide between the North Eastern Province, inhabited by Somalis, and the rest of the country is huge. As a last example, take India. In that country, the regional differences are on average much larger than the gender difference, but there are exceptions, such as the distance between Uttar Pradesh and Madhya Pradesh, two neighboring northern states.

\section{Specific cultural traits}

While our results suggest that the broad set of Facebook interests are able to provide an overall measure of culture, this section explores whether they can also capture specific cultural traits of interest to social scientists. To that end, we use a supervised machine learning algorithm that uses Facebook interests to predict close to 50 specific cultural traits or attributes, ranging from generosity to kinship tightness, from uncertainty avoidance to son bias, and from beef consumption to contraceptive use. The second column of Table S3 then reports 


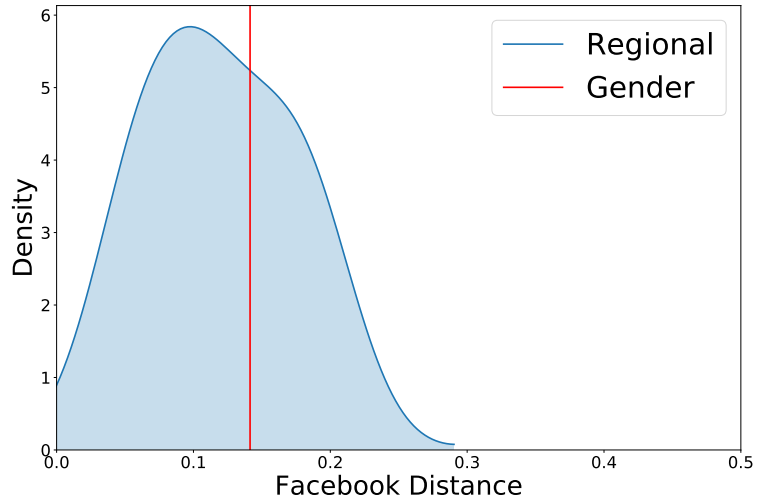

(a) Belgium

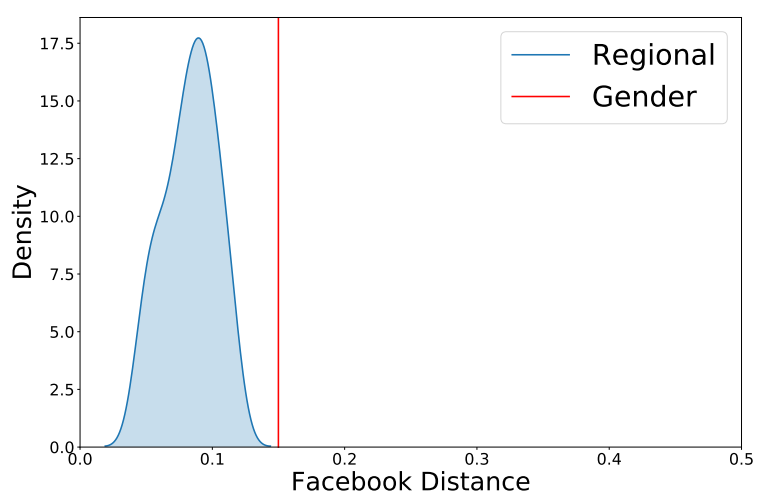

(c) Germany

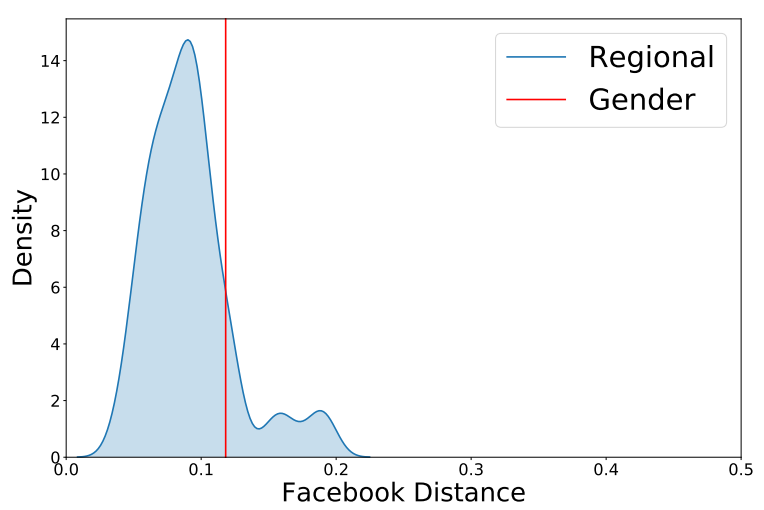

(e) Italy

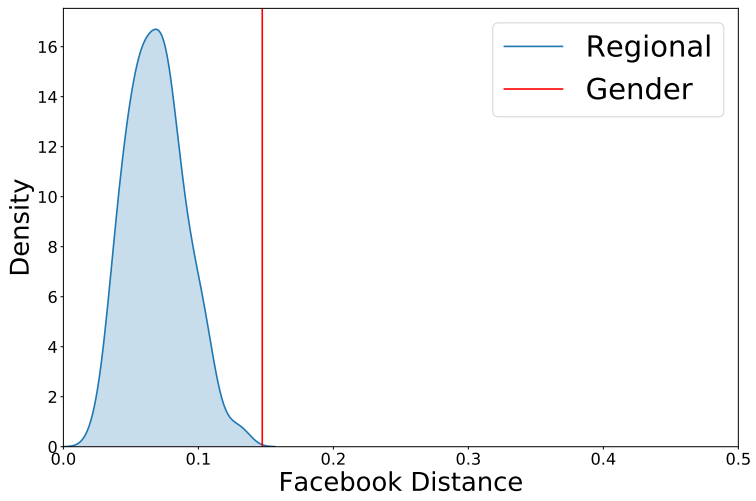

(b) France

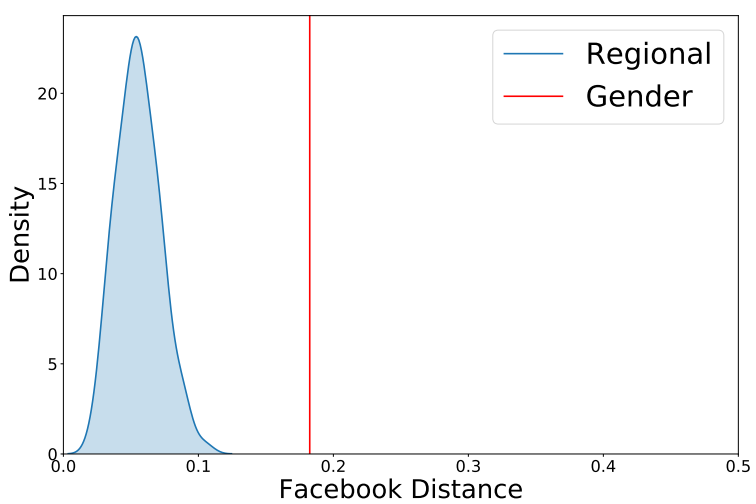

(d) Ireland

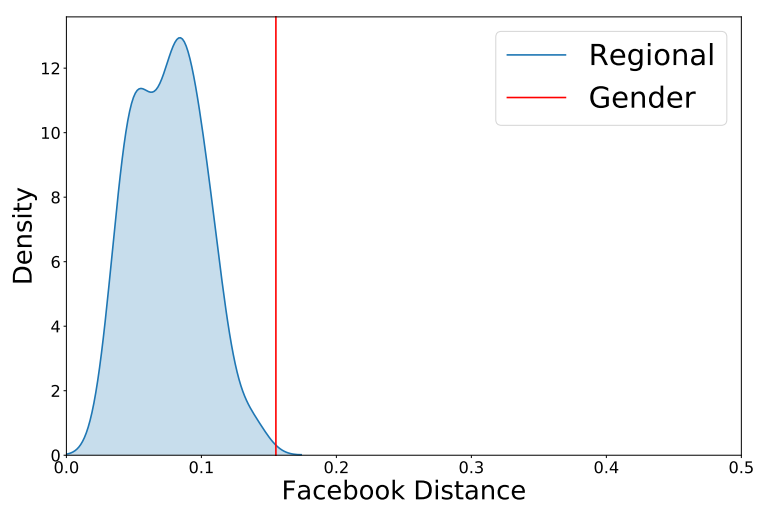

(f) Netherlands

Figure S12: Kernel density plots of interregional bilateral distances (blue) and gender distances (red). This figure displays histograms of bilateral distances between regions and bilateral distances between genders for 18 countries (BE: Belgium, BR: Brazil, DE: Germany, ES: Spain, FR: France, GB: Great Britain, IE: IN: India, Ireland, IT: Italy, KE: Kenya, MU: Mauritius, MX: Mexico, NG: Nigeria, NL: Netherlands, PT: Portugal, SV: El Salvador, TH: Thailand, US: United States). All figures have the same horizontal scale. 


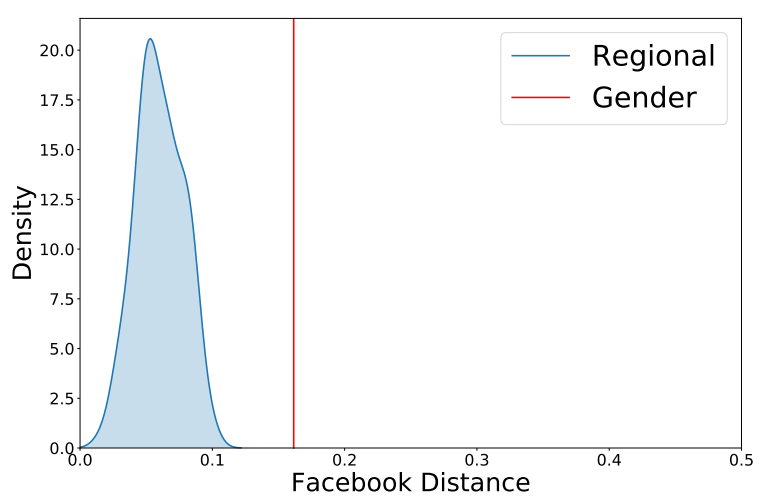

(a) Portugal

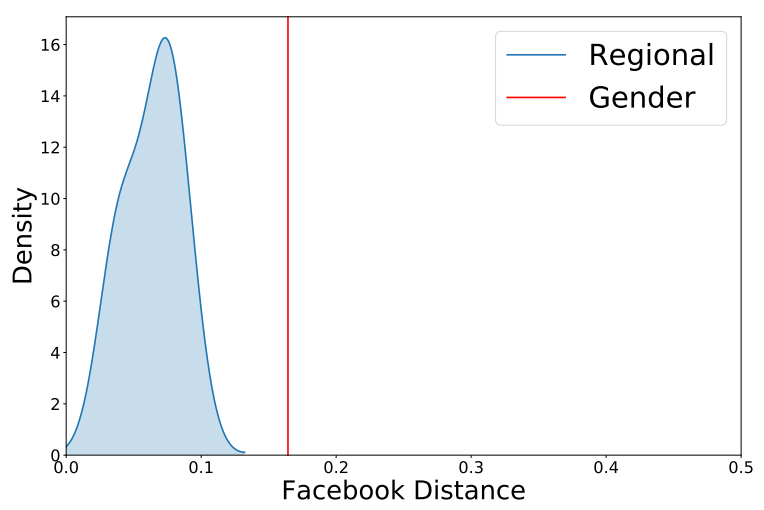

(c) Great Britain

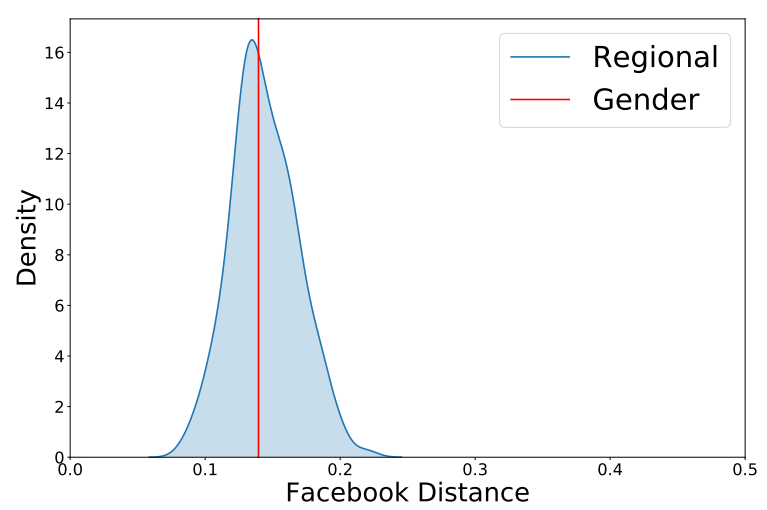

(e) Brazil

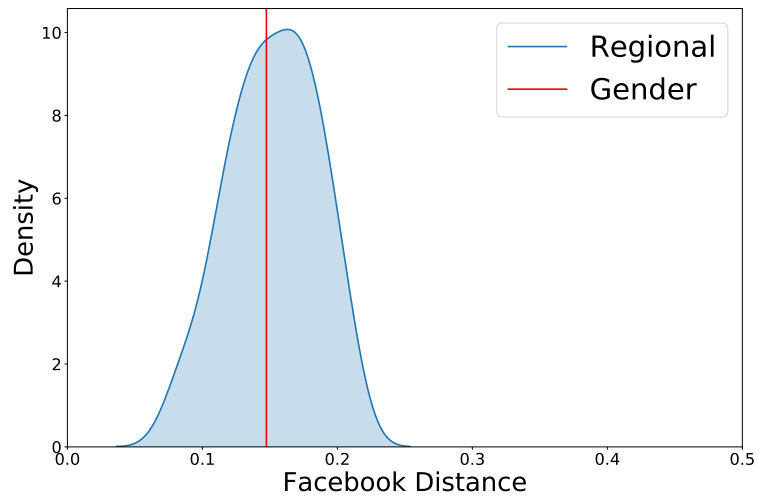

(b) Spain

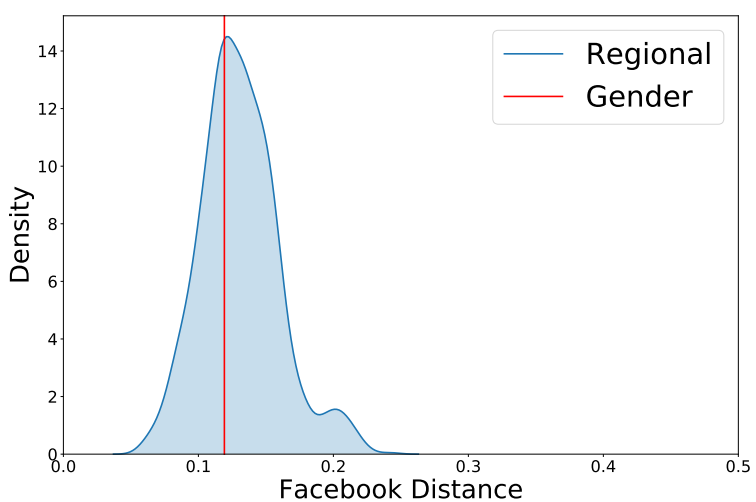

(d) United States

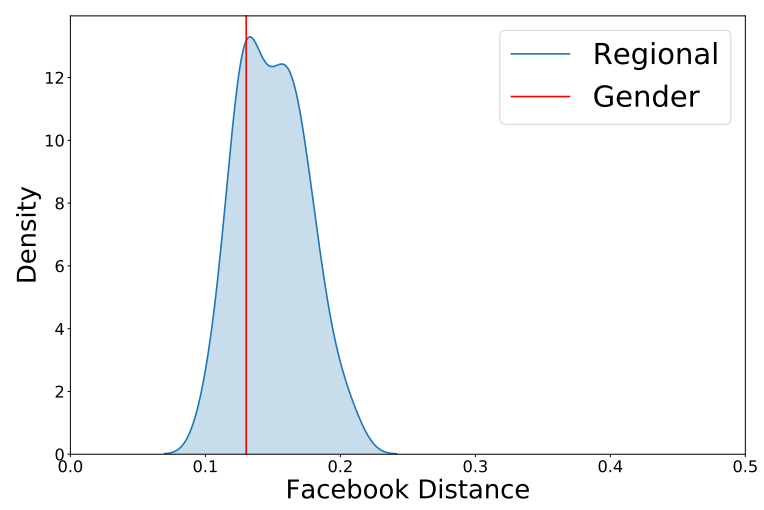

(f) El Salvador

Figure S12: Kernel density plots of interregional bilateral distances (blue) and gender distances (red) (continued). This figure displays histograms of bilateral distances between regions and bilateral distances between genders for 18 countries (BE: Belgium, BR: Brazil, DE: Germany, ES: Spain, FR: France, GB: Great Britain, IE: IN: India, Ireland, IT: Italy, KE: Kenya, MU: Mauritius, MX: Mexico, NG: Nigeria, NL: Netherlands, PT: Portugal, SV: El Salvador, TH: Thailand, US: United States). All figures have the same horizontal scale. 


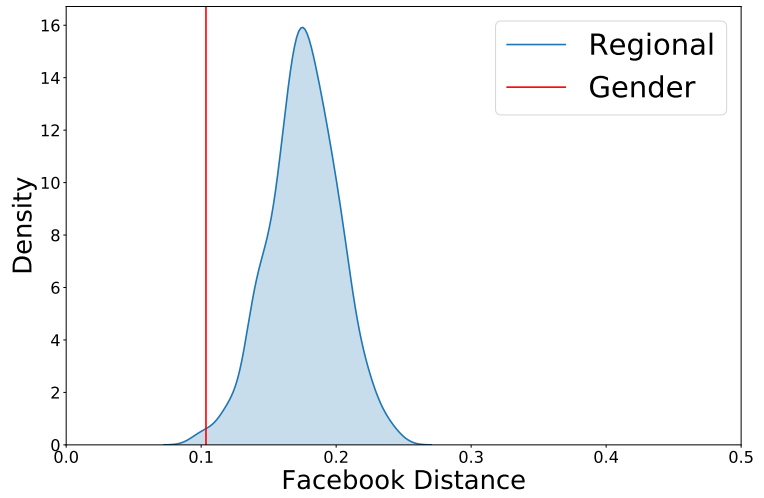

(g) Mexico

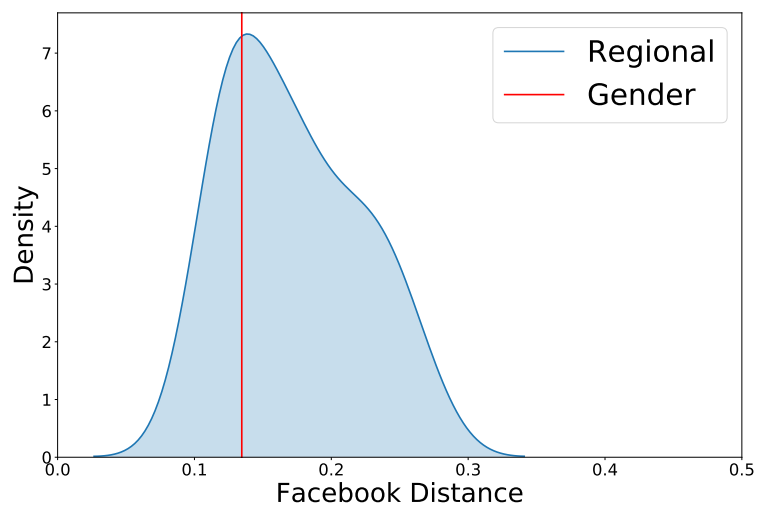

(i) Mauritius

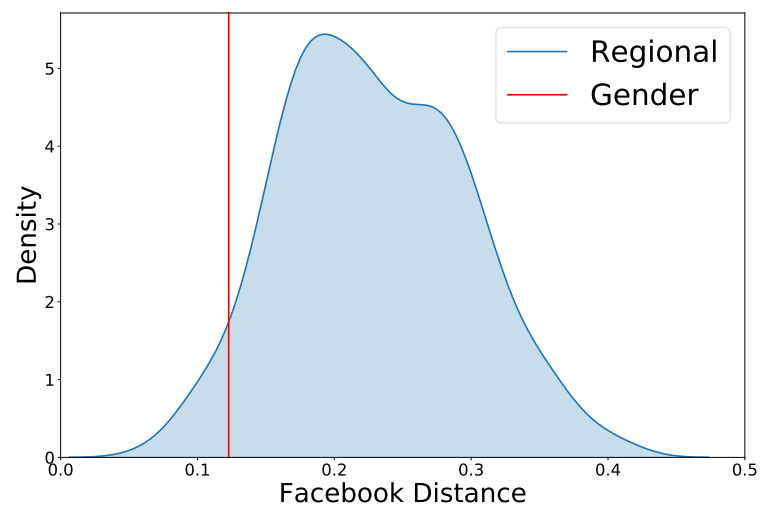

(k) India

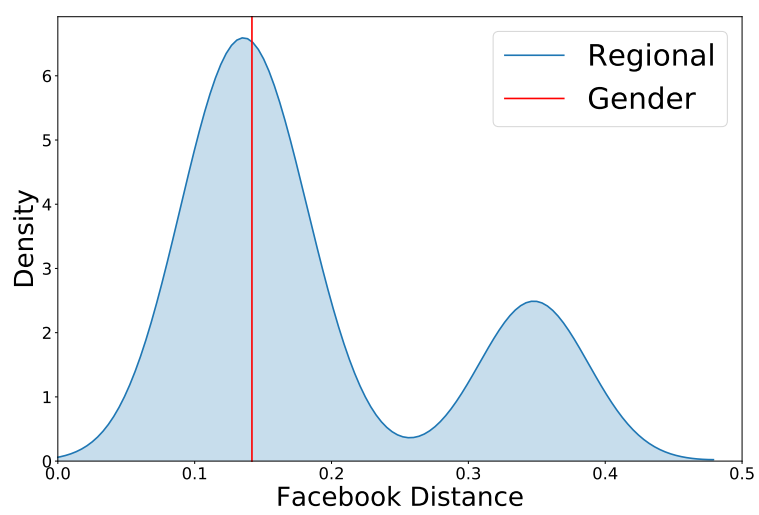

(h) Kenya

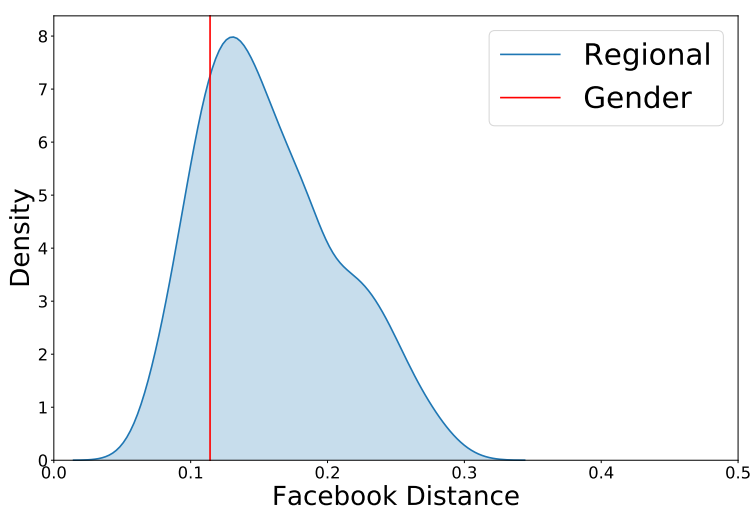

(j) Nigeria

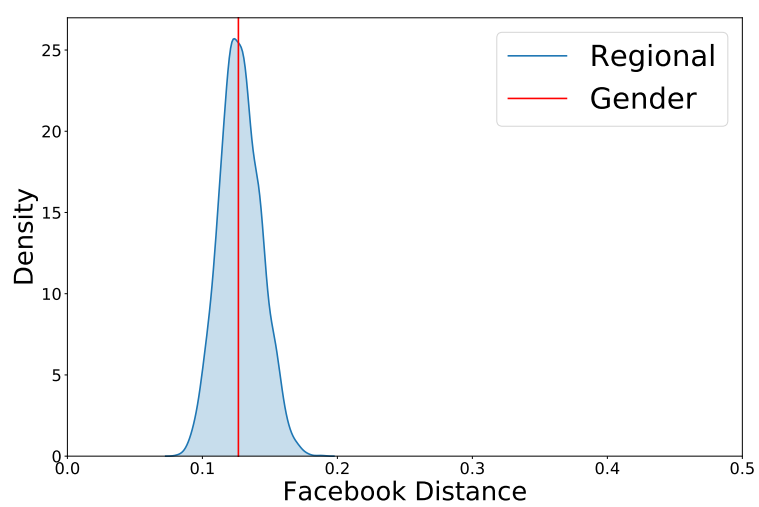

(1) Thailand

Figure S12: Kernel density plots of interregional bilateral distances (blue) and gender distances (red) (continued). This figure displays histograms of bilateral distances between regions and bilateral distances between genders for 18 countries (BE: Belgium, BR: Brazil, DE: Germany, ES: Spain, FR: France, GB: Great Britain, IE: IN: India, Ireland, IT: Italy, KE: Kenya, MU: Mauritius, MX: Mexico, NG: Nigeria, NL: Netherlands, PT: Portugal, SV: El Salvador, TH: Thailand, US: United States). All figures have the same horizontal scale. 
the correlations between the predicted cultural attributes and the observed cultural attributes. We find an average correlation of 0.59 , suggesting that the broad set of Facebook interests is able to capture specific, more traditional cultural traits, providing further validation of our measure.

To describe our approach in more detail, suppose we want to predict a country's degree of generosity. As inputs, we use the matrix of all Facebook interests and a vector of the degree of generosity from another data source (in this case, from the World Happiness Report). Using a training sample that consists of $90 \%$ of the countries, the Mathematica command "predict" then chooses among a set of standard supervised machine learning algorithms and predicts the degree of generosity in the test sample that consists of the $10 \%$ remaining countries. By running this algorithm 100 times on varying training samples, we obtain values for the average predicted generosity by country. We then correlate the generosity as predicted by Facebook to the generosity as observed in the data.

Alternatively, instead of using all Facebook interests to predict cultural traits, we can also use the principal components of the Facebook interests. The third column in Table S3 reports the correlations between predicted and observed cultural traits, based on the first 20 principal components. The correlations are slightly higher, at an average of 0.64 .

\section{Retrieving the cultural outliers of US regions}

This section provides added detail on Table 1 in the main text. Interestingly, Hawaii is the state with the most cultural outliers (147), over 2.5 times the number of outliers of the second highest state, Alaska (62). Further, the three states tied for third most cultural outliers (56) are Florida, Louisiana, and Texas. In contrast, the state with least cultural outliers is Pennsylvania (9) followed by Virginia (10) and Missouri (12).

Hawaii, the state with most cultural outliers, is the only state which is fully separated from continental North America. Further, Hawaii has strong cultural influence from other Pacific and Asian countries. There are several outlier cultural interests that show the clear influence of surf in the Hawaiian culture. We can find interests such as "Surf", "Windsurf", "Big Wave Surfing", "Surfer (magazine)" or "Surfline", as well as clothing brands using the surf concept as a marketing strategy: "Rip Curl", "QuickSilver", "Billabong". In the case of Alaska, a key cultural element is its relation with the Arctic, which makes Alaska different from other US states. We find explicit items such as "Arctic" and "Arctic Ocean", and other indirect items like "Magnetic Field" or "North Pole" among its cultural outliers. Looking at Louisiana, we discover multiple cultural outliers that are very related to one of the main fingerprints of this state: its Cajun cuisine. We find explicit interests such as "Cajun Cuisine", "Tabasco", and "Tabasco sauce" as cultural outliers of Louisiana. Furthermore, another important cultural element in New Orleans (the largest city) is Mardi Gras. Both "Mardi Gras" and "Carnival" appears as cultural outliers for Louisiana. In Utah, we find a cultural element highly linked to this state. Utah has more than two million Mormons, which is about one-third of the total number of Mormons in the United States. The cultural outliers of Utah capture this phenomenon and list several elements related to the Mormons: "Mormon", "Book of Mormon" and "Mormon Channel".

\section{Dendrogram with full sample of countries}

Figure S13 depicts the dendrogram from the main text, employing the full sample of Facebook countries. 


\begin{tabular}{|c|c|c|c|c|}
\hline $\begin{array}{l}\text { Cultural Dimension / } \\
\text { Sub-Dimension }\end{array}$ & $\begin{array}{l}\text { FB In- } \\
\text { terests }\end{array}$ & $\begin{array}{l}\mathbf{2 0} \\
\mathbf{P C}\end{array}$ & Obs & Source \\
\hline \multicolumn{5}{|c|}{ Demographic } \\
\hline Fertility rate & 0.85 & 0.88 & 156 & Fertility rate 2017, WDI, World Bank ${ }^{90}$ \\
\hline Age first marriage, men & 0.66 & 0.72 & 131 & Age first marriage men 2005-2014 (United Nations) ${ }^{91}$ \\
\hline Age first marriage, women & 0.73 & 0.79 & 147 & Age first marriage women 2005-2014 (United Nations) ${ }^{91}$ \\
\hline \multicolumn{5}{|c|}{ Food and Drinks } \\
\hline Alcohol, both sexes & 0.77 & 0.83 & 150 & Alcohol per capita, both sexes 2010-2016 (WHO) ${ }^{92}$ \\
\hline Alcohol, women & 0.79 & 0.85 & 150 & Alcohol per capita, female 2010-2016 (WHO) ${ }^{92}$ \\
\hline Alcohol, men & 0.78 & 0.83 & 150 & Alcohol per capita, male 2010-2016 (WHO) ${ }^{92}$ \\
\hline Beef consumption & 0.53 & 0.63 & 142 & Beef and buffalo consumption kg per capita $2013(\mathrm{FAO})^{93}$ \\
\hline Pork consumption & 0.84 & 0.88 & 138 & Pig consumption kg per capita $2013(\mathrm{FAO})^{93}$ \\
\hline Poultry consumption & 0.62 & 0.72 & 142 & Poultry consumption kg per capita $2013(\mathrm{FAO})^{93}$ \\
\hline Mutton and goat consumption & 0.52 & 0.55 & 142 & Mutton and goat consumption kg per capita $2013(\mathrm{FAO})^{93}$ \\
\hline Other meat consumption & 0.38 & 0.49 & 142 & Other meat consumption kg per capita $2013(\mathrm{FAO})^{93}$ \\
\hline \multicolumn{5}{|c|}{ Gender issues } \\
\hline Contraceptive use, women & 0.78 & 0.78 & 118 & Contraceptive use, women ages $15-49,2010-2019$, UNICEF $^{90}$ \\
\hline $\begin{array}{l}\text { Discriminatory work norms } \\
\text { against women }\end{array}$ & 0.78 & 0.81 & 148 & $\begin{array}{l}\text { Males } 15+\text { who agree it is unacceptable for women to work, 2016, } \\
\text { Georgetown GIWPS }\end{array}$ \\
\hline Gender inequality index, UN & 0.80 & 0.93 & 141 & Gender inequality index UN $2018^{95}$ \\
\hline Female employment & 0.67 & 0.62 & 149 & Employment women ages 25+, 2018, Georgetown GIWPS ${ }^{94}$ \\
\hline Gender gap & 0.72 & 0.73 & 134 & World Economic Forum Global Gender Gap, 2017-202096 \\
\hline Community safety, women & 0.57 & 0.60 & 148 & $\begin{array}{l}\text { Perception community safety among women ages } 15+, 2010-16 \text {, } \\
\text { Georgetown GIWPS }{ }^{94}\end{array}$ \\
\hline Intimate partner violence & 0.66 & 0.66 & 147 & $\begin{array}{l}\text { Intimate partner violence experienced by women, 2000-2017, } \\
\text { Georgetown GIWPS }^{94}\end{array}$ \\
\hline Son bias & 0.58 & 0.61 & 149 & Male to female ratio at birth, 2015-20, Georgetown GIWPS ${ }^{94}$ \\
\hline Women in parliament & 0.45 & 0.48 & 148 & Parliamentary seats held by women, 2019 , Georgetown GIWPS ${ }^{94}$ \\
\hline \multicolumn{5}{|c|}{ Wellbeing } \\
\hline Subjective well-being & 0.76 & 0.84 & 126 & Life ladder 2018 (World Happiness Report) ${ }^{97}$ \\
\hline Healthy life expectancy & 0.83 & 0.90 & 123 & Healthy life expectancy at birth 2018 (World Happiness Report) ${ }^{97}$ \\
\hline Insufficient physical exercise & 0.44 & 0.52 & 128 & Insufficient physical activity adults (\%), $2016(\mathrm{WHO})^{92}$ \\
\hline Freedom to make life choices & 0.44 & 0.55 & 126 & Freedom to make life choices 2018 (World Happiness Report) ${ }^{97}$ \\
\hline Generosity & 0.42 & 0.40 & 117 & Generosity 2018 (World Happiness Report) $^{97}$ \\
\hline Social support & 0.75 & 0.78 & 126 & Social support 2018 (World Happiness Report) ${ }^{97}$ \\
\hline Suicide rate & 0.56 & 0.61 & 148 & Crude suicide rates (per 100000 population) $2016, \mathrm{WHO}^{98}$ \\
\hline \multicolumn{5}{|c|}{ Society } \\
\hline Social capital & 0.75 & 0.77 & 129 & Social capital 2018-2019 (World Economic Forum) ${ }^{99}$ \\
\hline Relational mobility & 0.70 & 0.65 & 39 & Relational mobility Thomson et al PNAS ${ }^{28}$ \\
\hline Nepotism in business & 0.68 & 0.85 & 106 & Nepotism in business (Enke, based on Van de Vliert) ${ }^{100}$ \\
\hline Kinship tightness & 0.72 & 0.73 & 149 & Kinship tightness score (Enke) ${ }^{100}$ \\
\hline Nuclear family & 0.56 & 0.53 & 147 & $\begin{array}{l}\text { Nuclear family (Enke, based on Ethnographic Atlas and } \\
\text { Giuliano) }{ }^{100}\end{array}$ \\
\hline Perception of corruption & 0.62 & 0.72 & 121 & Perception of corruption 2018 (World Happiness Report) ${ }^{97}$ \\
\hline Ethnolinguistic fractionalization & 0.48 & 0.44 & 153 & ELF6 (Desmet, Ortuño and Wacziarg) ${ }^{26}$ \\
\hline \multicolumn{5}{|c|}{ Global Preferences Survey } \\
\hline Altruism & -0.15 & 0.12 & 72 & Altruism (Global Preferences Survey) ${ }^{101,102}$ \\
\hline Trust & 0.41 & 0.43 & 72 & Trust (Global Preferences Survey) ${ }^{101,102}$ \\
\hline Negative reciprocity & 0.04 & 0.06 & 72 & Negative reciprocity Global Preferences Survey) $)^{101,102}$ \\
\hline Positive reciprocity & 0.08 & 0.09 & 72 & Positive reciprocity (Global Preferences Survey) ${ }^{101,102}$ \\
\hline Risk taking & 0.33 & 0.37 & 72 & Willingness to take risks (Global Preferences Survey) ${ }^{101,102}$ \\
\hline Patience & 0.63 & 0.71 & 72 & Patience (Global Preferences Survey) ${ }^{101,102}$ \\
\hline \multicolumn{5}{|c|}{ Hofstede values } \\
\hline Indulgence vs restraint & 0.68 & 0.66 & 88 & Indulgence vs restraint (Hofstede) ${ }^{103}$ \\
\hline Uncertainty avoidance & 0.56 & 0.65 & 69 & Uncertainty avoidance index (Hofstede) $^{103}$ \\
\hline Long-term orientation & 0.77 & 0.82 & 89 & Long-term orientation vs short-term orientation (Hofstede) ${ }^{103}$ \\
\hline Masculinity vs femininity & 0.44 & 0.50 & 69 & Masculinity vs femininity (Hofstede) ${ }^{103}$ \\
\hline Individualism vs collectivism & 0.82 & 0.81 & 69 & Individualism vs collectivism (Hofstede) ${ }^{103}$ \\
\hline Power distance & 0.48 & 0.66 & 69 & Power distance index (Hofstede) ${ }^{103}$ \\
\hline
\end{tabular}

Table S3: Correlations between observed cultural traits and those same cultural traits as predicted by FB interests using a machine learning algorithm. Column 2 uses all FB interests in predicting, Column 3 uses the first 20 principal components of FB interests in predicting, and Column 4 gives the number of observations. 


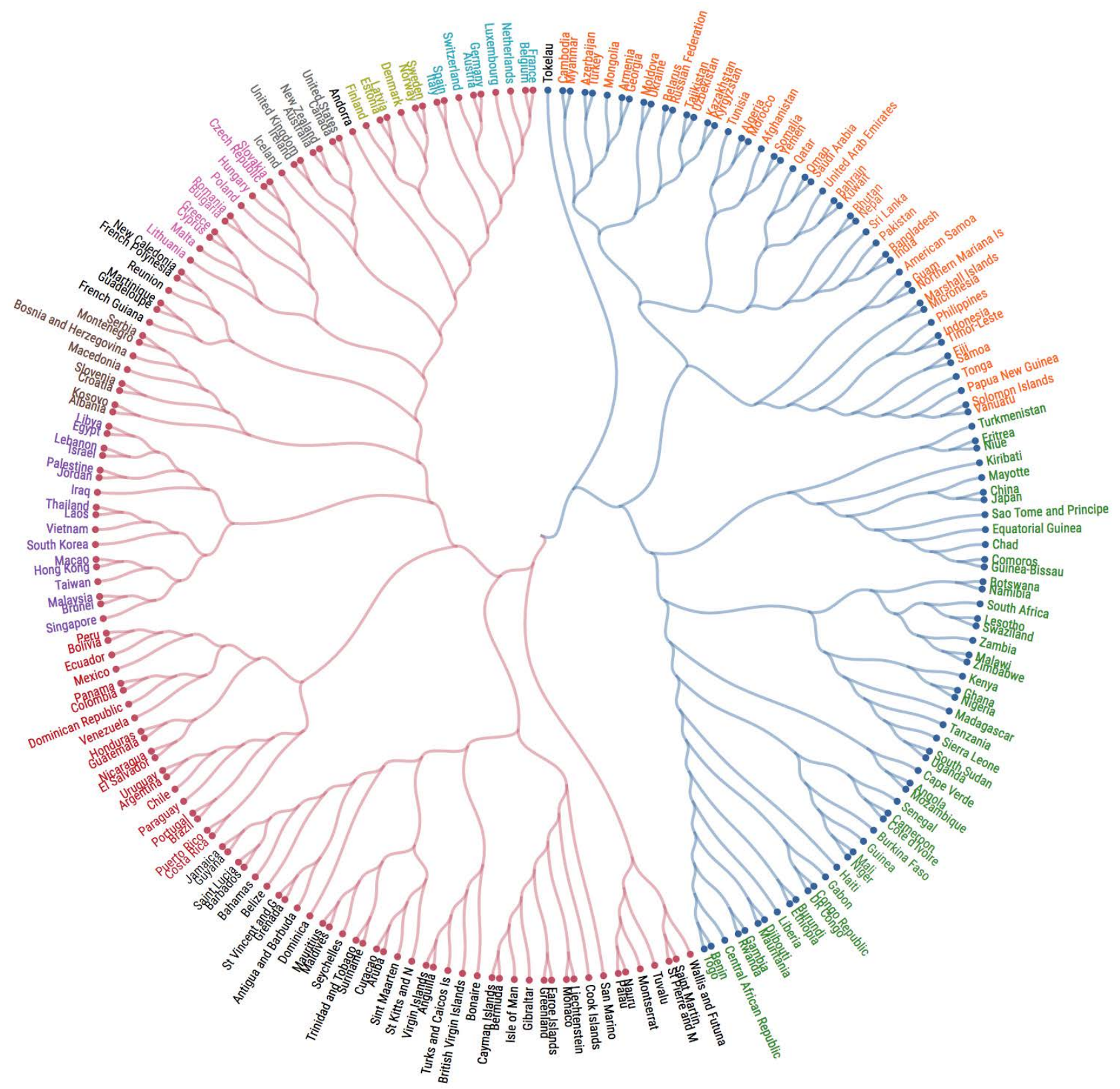

Figure S13: Hierarchical clustering of all in-sample countries based on Facebook distances. Dendrogram is generated using the cosine distance and Ward linkage method. All countries in the Facebook data are included. The color of a country's link represents its membership to a main cluster, while the color of its name represents its membership to a sub-cluster. Two countries of the same name color (respectively link color) are closer to each other than to a country of a different name (respectively link) color. 


\section{Examples of FB interests associated with different traditional and non-traditional cultural traits}

\begin{tabular}{|c|c|c|}
\hline Cultural trait & Category & Examples of FB Interests \\
\hline Arts & Traditional & $\begin{array}{l}\text { Abstract Art, African Art, Art Deco, Art museum, Art history, Art rock, Body art, Byzan- } \\
\text { tine Art, Ceramic Art, Conceptual art, Contemporary Art Gallery, Cooking art, Cover art, } \\
\text { Digital art, Fine-art photography, Folk art, Glass art, Gothic art, History of art, Interac- } \\
\text { tive art, Japanese art, Louvre Art Museum, Make Up Art, Medieval art, Metropolitan } \\
\text { Museum of Art, Mexican art, Modern art, Museum of Contemporary Art, Museum of } \\
\text { Modern Art, Nail Art, National Gallery of Art, New media art, Performance art, Pixel } \\
\text { art, Pop art, Public art, Red Ted Art, Sound art, Tattoos and Tattoo art, Thai Temple art } \\
\text { and architecture, Van Gogh Museum Art Museum. Video art, Visionary art, Wearable } \\
\text { art, Wood art, Latte art. }\end{array}$ \\
\hline Formalities & Traditional & $\begin{array}{l}\text { Arranged Marriage, Civil Marriage, Marriage (Catholic Church), Marriage in Islam, } \\
\text { Marriage license, Marriage vows, Civil procedure, Criminal procedure, Ritual, Ritual } \\
\text { purification, Rite, Rite Aid, Rite of passage, Prenuptial Agreement, Vehicle Registration } \\
\text { Plate. }\end{array}$ \\
\hline Religion & Traditional & $\begin{array}{l}\text { Religion, Afro-american Religion, Confession (religion), Glory (religion), Transcen- } \\
\text { dence (religion), Yoruba religion, Islam, Abraham in Islam, David in Islam, Five Pillars } \\
\text { of Islam, God in Islam, Jesus in Islam, Intimate parts (Islam), Islam is Beautiful, Islam } \\
\text { in the United States, Islam in India, Islam in Malaysia, Marriage in Islam, Shia Islam, } \\
\text { Shirk (Islam), Studying Islam, Sunni Islam, Women in Islam, Born again (Christian- } \\
\text { ity), Christianity, Disciple (Christianity), Early Christianity, Eastern Christianity, Eter- } \\
\text { nal life (Christianity), God in Christianity, Grace (Christianity), Heaven (Christianity), } \\
\text { Holy Spirit (Christianity), Minister (Christianity), Mission (Christianity), Passion (Chris- } \\
\text { tianity), Western Christianity, Judaism, Jewish culture, Jewish prayer, Conservative Ju- } \\
\text { daism, Messianic Judaism, Orthodox Judaism, Reform Judaism, Christian prayer, Mass } \\
\text { (liturgy). }\end{array}$ \\
\hline Politics & Traditional & $\begin{array}{l}\text { Politics, Centre-right politics, Far-left politics, Far-right politics, Gun politics in the } \\
\text { United States, Green politics, Law and order (politics), Left-wing politics, Opposition } \\
\text { (politics), Politics and Social Issues, Right-wing politics, Speaker (politics), Whip (pol- } \\
\text { itics), Brazilian Republican Party, Idaho Republican Party, Republican National Con- } \\
\text { vention, Republican National Committee, Republican Left of Catalonia, Republican } \\
\text { Party of Florida, Republican Party of Texas, Republican People's Party (Turkey), Re- } \\
\text { publican Party (United States), Democrat Party (Thailand), Brazilian Democratic Move- } \\
\text { ment Party, Christian Democratic Party (Chile), Christian Democratic People's Party } \\
\text { of Switzerland, Christian Democratic Union (Germany), Idaho Democratic Party, Lib- } \\
\text { eral Democratic Party (Japan), Liberal Democracy, Democratic Party (United States), } \\
\text { Democratic Socialism, Socialism, Communism, Brazilian Socialist Party, Italian Social- } \\
\text { ist Party, Spanish Socialist Worker's Party, Barack Obama, Donald Trump, Bill Clinton, } \\
\text { Hillary Clinton. }\end{array}$ \\
\hline $\begin{array}{c}\text { Social } \\
\text { Structures }\end{array}$ & Traditional & $\begin{array}{l}\text { Family, Marriage, Town hall, Municipality, State, Government, Executive (government), } \\
\text { Church service, Social Security, Police, Army, Non-Governmental Organisation. }\end{array}$ \\
\hline Angry Birds & Non-Traditional & $\begin{array}{l}\text { Angry Birds, Angry Birds (video game), Angry Birds } 2 \text { Angry Birds Epic, Angry Birds } \\
\text { POP! -Bubble Shooter, angry birds friends }\end{array}$ \\
\hline Soccer fans & Non-Traditional & $\begin{array}{l}\text { Real Madrid C.F, History of Real Madrid C.F., Real Madrid Fans, Real Madrid Fans } \\
\text { Club, FC Barcelona, FC Barcelona Fans Club, F.C. United of Manchester, Manchester } \\
\text { City F.C, Manchester City F.C. Supporters. }\end{array}$ \\
\hline Loungewear & Non-Traditional & Tracksuit, Shorts, Bermuda shorts, Boxer shorts, T-shirt, Sports bra. \\
\hline Running & Non-Traditional & $\begin{array}{l}\text { Running, Barefoot running, Cross country running, Long-distance running, Middle- } \\
\text { distance running, Mizuno running North America, Road running, Sprint (running), Sta- } \\
\text { dion (running race), Trail running, Women's Running Magazine, Adidas running, Brooks } \\
\text { running, Ironman Triathlon, Half marathon, Marathon }\end{array}$ \\
\hline
\end{tabular}

Table S4: Table mapping FB interests to cultural traits both used in traditional measures of culture and nontraditional ones. This table illustrates actual FB interests included in the interest set used in the paper that can be mapped into specific cultural traits. We have selected nine cultural traits in this table divided into two groups. First, cultural traits that are usually employed in traditional measures of culture (arts, formalities, religion, politics and social structures). Second, traits that our measure of culture also captures but are not used in traditional measures of culture (angry birds, football fans, loungewear and running). 